\author{
UNIVERSIDADE DE SÃO PAULO \\ INSTITUTO DE FÍSICA
}

\title{
Estática e Dinâmica do Modelo Gaussiano
}

\author{
Eduardo do Carmo
}

Orientador: Prof. Dr. Silvio Roberto de Azevedo Salinas

\section{BANCA EXAMINADORA}

Prof. Dr. Silvio Roberto de Azevedo Salinas (IFUSP)

Prof. Dr. Carlos Seihiti Orii Yokoi (IFUSP)

Prof. Dr. Jügen Fritz Stilck (UFF)

São Paulo 


\section{Agradecimentos}

Tratai essas pessoas com consideração, bem como todos aqueles que ajudam e trabalham na mesma obra.

(1 Cor 16,16)

- ao professor Silvio Roberto de Azevedo Salinas, por ter me concedido a honra de ser seu aluno, pela valorosa orientação, que envolveu muito mais que discussões sobre física, por seu incentivo, por seu exemplo e por sua paciência.

- a minha família, que é meu alicerce, pelo incentivo, por me fazerem perseverar e por compreenderem minha ausência.

- a todos os amigos, aos irmãos de república, de agora e do passado, de modo maior ao Prof. Dr. Juliano, que tanto me ajudou e ao Ronaldo, pela ajuda com o português.

- ao todos os professores do grupo de Mecânica Estatística, aos funcionários do DFG e aos mestres da UEM, em especial ao Renio.

- a todos os companheiros de empreitada do corredor, em especial ao Danilo, por sua ajuda constante, ao Rodrigo, e aos companheiros da sala 306, aprendi muito com todos.

- ao CNPQ, pelo apoio financeiro. 


\section{Resumo}

O modelo gaussiano para um ferromagneto em uma rede hipercúbica d-dimensional é apresentado num contexto estático e dinâmico. Sua termodinâmica é investigada calculando diversas grandezas para a faixa de temperatura na qual o modelo é definido $\left(T \geq T_{c}\right)$. Expoentes críticos e a dimensão crítica são definidos e calculados para o caso ferromagnético com interações de primeiros vizinhos.

Dois tipos de dinâmica são inseridos no modelo gaussiano: a dinâmica de Langevin e a de Cahn-Hilliard. São calculadas a função de auto-correlação e a função resposta, que são os observáveis de interesse num enfoque dinâmico. A maneira como esses observáveis se relacionam também é investigada, via teorema de flutuação-dissipação. De maneira mais geral, um eventual comportamento dinâmico, tipo Langevin, é estudado através do formalismo MSR. Sendo a técnica MSR apropriada ao estudo de sistemas com desordem, calculam-se as funções de auto-correlação e resposta de um modelo gaussiano na presença de um campo aleatório (desordem do tipo quenching).

Finalmente, de maneira independente e como um exercício de mecânica estatística, três modelos são apresentados: um modelo de gás de rede com graus de liberdade orientacionais do tipo Ising, o modelo de Potts de q estados e o modelo de Maier-Saupe para um cristal

líquido. É mostrado que esses modelos, de certa maneira, estão próximos. Em uma maneira mais explícita, numa versão de campo médio, mostra-se que a energia livre de um modelo de Potts de três estados e uma versão discretizada para o modelo de MaierSaupe, com restrições às direções do diretor, possuem a mesma energia livre. 


\section{Abstract}

The Gaussian model of a ferromagnet on a d-dimensional hipercubic lattice is presented, in the static and dynamic contexts. The thermodynamics of the Gaussian model is investigated evaluating several thermodynamic quantities, in the temperature range of validity of the model $\left(T \geq T_{c}\right)$. For a ferromagnetic model with first neighbour interactions, the critical dimension and the critical exponents are found.

Two kinds of dynamics are applied on the Gaussian model: the Langevin dynamics and the Cahn-Hilliard dynamics. The auto-correlation and the response functions, which are the interesting observables from the dynamical point of view, are calculated. The relation between these two functions is also analyzed, through the fluctuation-dissipation theorem. In a more general way, the Langevin behavior is studied through the MSR formalism, which is useful for systems with disorder. The MSR formalism is applied to find auto-correlation and response functions of a random-field Gaussian model (quenched disorder).

Furthermore, as a statistical mechanics exercise, three models are presented: a lattice gas with Ising-like orientational degrees of freedom, the q-state Potts model, and the Maier-Saupe model for the transitions in a nematic liquid crystal. At the mean field level, we show that these three models are similar. In particular, we show that the free energy of the three-state Potts Model is equal of the free energy of a discretized version of the Maier-Saupe model, with restrictions on the directions of the director. 


\section{Sumário}

1 Introdução 1

2 A termodinâmica do modelo gaussiano $\quad 7$

2.1 Propriedades termodinâmicas . . . . . . . . . . . . . . . 9

2.2 Modelo ferromagnético com interações de primeiros vizinhos . . . . . . . . 10

2.3 Análise da energia interna por spin para $d=1 \ldots \ldots \ldots \ldots$

2.4 Função de correlação de pares de spins . . . . . . . . . . . . . . . . . . 17

3 Comportamento dinâmico $\quad 19$

3.1 Modelo gaussiano crítico na dinâmica de Langevin . . . . . . . . . . . . . . 19

3.2 Modelo gaussiano crítico na dinâmica de Cahn-Hilliard . . . . . . . . . 26

3.3 Formalismo MSR . . . . . . . . . . . . . . . . . . . . . 30

3.3 .1 O método . . . . . . . . . . . . . . . . 31

3.3.2 Introdução dos campos de Grassmann . . . . . . . . . . . . . . . 36

3.4 O modelo Gaussiano segundo o formalismo MSR . . . . . . . . . . . . . . 39

3.5 O modelo gaussiano na presença de um campo aleatório . . . . . . . . . . . 42

3.5.1 Aplicação da técnica MSR . . . . . . . . . . . . . . . . . 46

4 Modelos de spin para mesofases cristalinas 53

4.1 O gás de rede . . . . . . . . . . . . . . . . . . 54

4.1.1 Gás de rede com graus orientacionais de liberdade . . . . . . . . . 56

4.2 O modelo de Potts . . . . . . . . . . . . . . . . . . . 58

4.3 O modelo de Maier-Saupe . . . . . . . . . . . . . . . . . . . 62

4.3.1 Parâmetro de ordem mais geral . . . . . . . . . . . . . 65 
4.3.2 Modelo de Maier-Saupe discretizado . . . . . . . . . . . . . . 68

4.3.3 Algumas comparações . . . . . . . . . . . . . . . . . . . . 70

5 Considerações finais

A Teorema de flutuação-dissipação

B Integrais Gaussianas

Referências Bibliográficas 


\section{Lista de Figuras}

2.1 Contorno no plano complexo . . . . . . . . . . . . . . . . 12

4.1 Alças de Van der Waals . . . . . . . . . . . . . . . . 56

4.2 Vetores do modelo de Potts . . . . . . . . . . . . . . . . 60

4.3 Modelo de Potts três estados . . . . . . . . . . . . . . . . . . . 61

4.4 Cristal líquido nemático . . . . . . . . . . . . . 63 


\section{Capítulo 1}

\section{Introdução}

Os sistemas magnéticos constituem um dos melhores laboratórios para o desenvolvimento e teste de modelos estatísticos. O carro-chefe desses modelos foi proposto por W. Lenz (1888 - 1957) em 1920 e tratado quantitativamente, em uma dimensão, por seu aluno Ernst Ising (1900 - 1998) em 1924. O cálculo da função de partição a campo nulo do modelo de Ising, numa rede quadrada com interações de primeiros vizinhos, foi realizado por Lars Onsager (1903-1976) em 1944, constituindo um dos feitos importantes da física teórica.

O modelo de Ising é definido pelo hamiltoniano

$$
\mathcal{H}_{N}=-\frac{1}{2} \sum_{\vec{r}, \vec{r}^{\prime}} J_{\vec{r}, \vec{r}^{\prime}} S_{\vec{r}} S_{\vec{r}^{\prime}}-\sum_{\vec{r}} H_{\vec{r}} S_{\vec{r}}
$$

onde $\vec{r}$ designa um sítio de uma rede cúbica com $d$ dimensões, $S_{\vec{r}}= \pm 1$ é a variável de spin no sítio $\vec{r}, J_{\vec{r}, \vec{r}^{\prime}}$ é o parâmetro de troca que media as interações entre os spins dos sítios $\vec{r}$ e $\vec{r}^{\prime}$, e $H_{\vec{r}}$ é um campo externo aplicado ao spin no sítio $\vec{r}$.

Desde o seu surgimento, o modelo de Ising tem sido empregado em muitas outras situações físicas além do magnetismo, como na modelagem de ligas binárias e de misturas de dois tipos diferentes de moléculas. No entanto, apesar de seu universo de atuação ser muito vasto, atraindo assim a atenção de muitos físicos durante mais de 80 anos de existência, pouco se avançou, sob o ponto de vista analítico, desde a solução de Onsager. Embora existam resultados numéricos muito bons para o modelo de Ising tridimensional e para o modelo de Ising bidimensional na presença de um campo externo, eles ainda permanecem insolúveis sob o ponto de vista analítico. 
Motivado pelos avanços de Onsager, M. Kac $(1914$ - 1984) propôs em 1947, como simplificação matemática, os modelos gaussiano e esférico. Esses modelos, que possuem caráter essencialmente matemático, sempre gozaram de muito prestígio desde sua definição. Ainda que tais molelos sejam demasiadamente abstratos, eles possuem soluções exatas (Berlin e Kac, 1952) enquanto o modelo de Ising resiste a um tratamento analítico. O prestígio dos modelos esférico e gaussiano ainda se justifica por servirem como parâmetros da robustez de técnicas usadas no estudo das transições de fase, no caso do modelo esférico, e ponto de partida de expansões perturbativas num contexto de grupo de renormalização e teorias de campos, no caso do modelo gaussiano. Eles também se caracterizam pela sua importância sob o ponto de vista didático.

A idéia por trás dos modelos gaussiano e esférico está em substituir o vínculo forte $S_{\vec{r}}^{2}=1$ por outro vínculo mais frágil. Tais modelos são definidos pelo mesmo hamiltoniano de Ising, porém os spins passam a ser variáveis que podem assumir qualquer valor na reta real. Para garantir a convergência de suas funções de partição, diferentes vínculos são adicionados. No caso do modelo gaussiano, exige-se que os spins contínuos sejam obedientes a uma distribuição gaussiana. No caso do modelo esférico, a soma dos quadrados de todos os spins deve ser igual ao número de spins da rede. De acordo com uma interpretação geométrica do cálculo da função de partição, a soma sobre todos os estados possíveis do modelo gaussiano é equivalente a uma integração sobre todo o hiper-espaço d-dimensional dos possíveis valores das variáveis $S_{\vec{r}}$; para o modelo esférico, devemos realizar integrações sobre a hiperesfera de raio $N^{d / 2}$, onde $N^{d}$ é o número de spins da rede. De certa forma o modelo esférico está mais próximo do modelo de Ising.

Em virtude da extensa aplicabilidade do modelo gaussiano e da possibilidade de sua investigação através de todas as técnicas usadas no estudo de fenômenos críticos, apesar de não ser definido abaixo de sua temperatura crítica, nós o elegemos como laboratório de estudo, investigando a sua termodinâmica e introduzindo, de forma suplementar, um comportamento dinâmico. Não se deve concluir precocemente que, como esse modelo não é definido abaixo de $T_{c}$, ele não possua nada de interessante, que é o que vamos mostrar.

O estudo do comportamento dinâmico do modelo gaussiano, que é originalmente definido apenas no equilíbrio, parece um tanto contraditório. Porém, é possível inserir 
comportamentos dinâmicos nesses modelos estatísticos de spins. Isso é feito sem muitas restrições, segundo a física do problema, isto é, escolhendo a dinâmica que melhor se adapta à situação que se deseja investigar. As diversas dinâmicas que podem ser utilizadas em mecânica estatística de não-equilíbrio foram classificadas em celebrado trabalho de P. C. Hohenberg e B. I. Halperin de 1977 (Hohenberg e Halperin, 1977). Dentre os vários tipos de dinâmica, a do tipo $A$ é a mais difundida entre os físicos, sendo uma dinâmica dissipativa representada pela equação de movimento de Langevin, que por sua vez surgiu no contexto do movimento browniano. Todavia, outro tipo de dinâmica, famosa entre os metalurgistas e não menos importante, é a do tipo $B$, que se propõe a descrever o comportamento dinâmico de sistemas cujo parâmetro de ordem é conservado. A dinâmica do tipo B é representada pela equação de Cahn-Hilliard.

Ultimamente tem havido interesse em verificar se certas propriedades do estado de equilíbrio - como o teorema de flutuação-dissipação - ainda continuam válidas numa situação fora do equilíbrio. O teorema de flutuação-dissipação (TFD) é sempre válido num cenário estático, relacionando a magnetização com a suscetibilidade em sistemas magnéticos. Considerando os observáveis análogos à magnetização e à suscetibilidade, que num contexto de não-equilíbrio são a função de auto-correlação e a função resposta, pode-se verificar a validade do TFD numa forma modificada. Quando o TFD não é verificado em sua forma clássica, há uma indicação de que o mecanismo que relaciona as flutuações térmicas com a dissipação de energia é diferente daquele verificado no equilíbrio e previsto pela teoria da função resposta linear. De acordo com os trabalhos de Cugliandolo e Kurchan, a verificação de uma forma modificada do TFD em algumas situações pode ser explicada através da introdução de uma temperatura efetiva. Esses fatos vêm fornecendo "fôlego" para um grande número de trabalhos nos últimos anos (Crisanti e Ritort, 2003; Cugliandolo, 2002; Cugliandolo e Kurchan, 1993).

Um problema formidável de mecânica estatística de não-equilíbrio surgiu quando se iniciou o estudo da evolução temporal de sistemas do tipo vidro de spin. As complicações vieram pelo fato desse tipo de sistema ser modelado através de variáveis aleatórias, que se distribuem segundo uma dada densidade de probabilidade. Diz-se, então, que esses problemas possuem desordem. Quando as variáveis desordenadas (campos externos, interações 
de troca, etc) permanecem constantes enquanto as variáveis de spin flutuam tem-se uma desordem do tipo quenched. O estudo de sistemas no equilíbrio que possuem esse tipo de desordem pode ser feito através do método das réplicas. Em um cenário dinâmico, De Dominicis em 1978 substituiu a aplicação desse método por um procedimento mais físico, utilizando um formalismo proposto por P. C. Martin, E. Siggia e H. Rose (Martin, Siggia, e Rose, 1973). Esse procedimento engenhoso substitui o método das réplicas, pois define uma função geratriz devidamente normalizada. Modelos magnéticos com acoplamentos desordenados (como os vidros de spin) ou na presença de um campo aleatório vêm exercendo um grande fascínio sobre os físicos estatísticos, sobretudo por apresentarem diagramas de fase muito ricos, com a potencialidade de descrever fases vítreas.

A nossa dissertação se divide em duas partes. A primeira parte aborda os tópicos comentados nos parágrafos precedentes e a segunda trata de semelhanças entre modelos bem conhecidos na literatura. Na verdade a segunda parte é um exercício de mecânica estatística, servindo de base às investigações que pretendemos realizar futuramente nos domínios dos cristais líquidos e dos cristais plásticos.

Desde cedo nos ensinam que a matéria se apresenta em três formas: sólida, líquida e gasosa. Porém, existem materiais que em determinadas situações apresentam propriedades da fase sólida e em outras se comportam como líquidos: os cristais líquidos e os cristais plásticos. Tais materiais podem ser obtidos a partir da dissolução de um material sólido cristalino em um solvente. Dessa maneira, em determinadas condições de temperatura, concentração e pressão, podem surgir essas "mesofases".

Em trabalho anterior no nosso grupo (Tsai, 1994), com a finalidade de explicar o diagrama de fases de um cristal plástico, foi detalhadamente analisada uma versão de campo médio de um gás de rede com graus orientacionais de liberdade do tipo Ising. Na época também se sabia que talvez fosse mais apropriado utilizar um gás de rede com variáveis de Potts a fim de representar com maior fidelidade os graus orientacionais de liberdade. Recentemente percebemos que seria ainda mais interessante e apropriado representar os graus orientacionais de liberdade do gás de rede através de um modelo de Maier-Saupe, que tem sido amplamente utilizado no estudo das transições de fase em cristais líquidos do tipo nemático. 
Motivados por esses estudos anteriores, decidimos inicialmente recuperar os cálculos para o gás de rede acoplado a variáveis orientacionais do tipo Ising. Em seguida, consideramos a versão de campo médio do modelo de Potts de $q$ estados, prestando maior atenção ao caso $q=3$. Depois disso, estudamos um versão discretizada do modelo de Maier-Saupe, mostrando que ela se caracteriza pela mesma expressão da energia livre do modelo de Potts de $q=3$ estados (que não é muito diferente da expressão da energia livre do gás de rede com variáveis orientacionais do tipo Ising). O problema mais envolvido, do gás de rede com variáveis de Maier-Saupe, será deixado para um trabalho posterior.

Essa dissertação está organizada em uma introdução (cap.1) e três capítulos. No segundo capítulo definimos o modelo gaussiano e discutimos as suas propriedades termodinâmicas (seção 2.1). As duas seções seguintes tratam de particularizações para o caso ferromagnético com interações entre primeiros vizinhos em uma rede d-dimensional, incluindo uma análise de um modelo unidimensional. Por fim, apresentamos um cálculo das funções de correlação de pares de spin desse modelo (seção 2.3). Tais funções são calculadas para um sistema no ponto crítico e em seguida em suas proximidades.

O terceiro capítulo contém o estudo de um eventual comportamento dinâmico do modelo gaussiano. Essa investigação é feita usando as dinâmicas de Langevin (seção 3.1) e de Cahn-Hilliard (seção 3.2). Nessas seções são calculados os observáveis de interesse dinâmico: funções de autocorrelação e resposta. Ainda analisamos como essas funções se relacionam nas proximidades do ponto crítico (teorema de flutuação-dissipação). Em seguida, apresentamos o procedimento mais físico para o estudo de um comportamento dinâmico segundo o formalismo MSR. Após a apresentação do método, introduzimos uma versão envolvendo variáveis fermiônicas (seção 3.3). Aplicamos esse formalismo ao modelo gaussiano definido no capítulo segundo e também a um modelo gaussiano na presença de um campo aleatório. A técnica MSR é ideal para tratar sistemas com desordem (seções 3.4 e 3.5$)$.

Finalmente, no quarto capítulo estudamos um modelo de gás de rede generalizado para conter graus de liberdade orientacionais tipo Ising (seção 4.1), e um modelo de Potts de q estados (seção 4.2). Na seção 4.3 apresentamos o modelo de Maier-Saupe para transições de fase em cristais líquidos nemáticos. Enfatizamos a necessidade da construção de um 
parâmetro de ordem mais geral para a investigação das propriedades termodinâmicas desses materiais. Apresentamos generalizações gradativas do modelo de Maier-Saupe, que culminam na construção de uma energia livre para um modelo com restrições nas direções do diretor (seção 4.3.2). Mostramos que a essa energia livre desse modelo, a menos de alguns fatores, é igual à energia livre da versão de campo médio de um modelo de Potts de três estados.

Essa dissertação ainda possui dois apêndices. O primeiro traz uma formulação para o teorema de flutuação-dissipação e sua extensão a sistemas que estejam evoluindo no tempo. O segundo apêndice apresenta formas generalizadas para as integrais gaussianas, que são usadas extensamente durante a dissertação. 


\section{Capítulo 2}

\section{A termodinâmica do modelo}

\section{gaussiano}

O modelo gaussiano é definido pelo hamiltoniano (1.1) com a adição de um fator de convergência. Assim, temos a função canônica de partição do modelo gaussiano,

$$
Z_{G}(\beta)=\int_{\mathcal{R}} \exp \left\{\frac{\beta}{2} \sum_{\vec{r}, \vec{r}^{\prime}} J_{\vec{r}, \vec{r}^{\prime}} S_{\vec{r}} S_{\vec{r}^{\prime}}-\frac{b}{2} \sum_{\vec{r}} S_{\vec{r}}^{2}\right\} \prod_{\vec{r}} d S_{\vec{r}}
$$

onde $b>0$ é o peso gaussiano de convergência. O cálculo dessa função de partição é feito através de uma transformação de variáveis que diagonaliza a forma quadrática do argumento da exponencial.

Vamos definir o vetor de um sítio do espaço real,

$$
\vec{r}=r_{1} \hat{a}_{1}+r_{2} \hat{a}_{2}+\cdots+r_{d} \hat{a}_{d}
$$

e seu correspondente no espaço recíproco,

$$
\vec{q}=q_{1} \hat{b}_{1}+q_{2} \hat{b}_{2}+\cdots+q_{d} \hat{b}_{d}
$$

onde $r_{i}$ e $q_{i}$ são inteiros e as bases no espaço real e no espaço de Fourier são ortogonais, obedecendo a relação

$$
\hat{a}_{i} \cdot \hat{b}_{j}=2 \pi \delta_{i j}
$$

onde $\delta_{i j}$ designa o delta de Kronicker. 
Supondo que cada direção da rede cristalina possua $N$ sítios, com espaçamento $a$ entre eles, os vetores permitidos na primeira zona de Brillouin são dados por

$$
\vec{q}=\frac{2 \pi}{L}\left(q_{1}, q_{2}, \cdots, q_{d}\right)
$$

onde $-L / 2<q_{1}, q_{2}, \cdots, q_{d} \leq L / 2$.

A transformação discreta de Fourier das variáveis de spin é definida através da relação

$$
S_{\vec{r}}=\frac{1}{N^{\frac{d}{2}}} \sum_{\vec{q}} \hat{S}_{\vec{q}} e^{i \vec{q} \cdot \vec{r}}
$$

onde a soma deve ser realizada sobre a primeira zona de Brillouin (Yokoi, 1978).

Para não sobrecarregar a notação vamos deixar de explicitar o caráter vetorial das variáveis que localizam os sítios, tanto no espaço real quanto no recíproco.

Aplicando a transformada de Fourier ao argumento da exponencial da função de partição e supondo que a hiper-rede esteja sujeita a condições periódicas de contorno (o que não terá conseqüências no limite termodinâmico), temos

$$
\begin{aligned}
Q & =\frac{\beta}{2} \sum_{r, r^{\prime}} J\left(\left|\vec{r}-\vec{r}^{\prime}\right|\right) S_{r} S_{r^{\prime}}-\frac{b}{2} \sum_{r} S_{r}^{2} \\
& =\frac{1}{2} \sum_{q}[\beta \hat{J}(q)-b] \hat{S}_{q} \hat{S}_{-q},
\end{aligned}
$$

onde

$$
\hat{S}_{q}=\frac{1}{N^{d / 2}} \sum_{r} S_{r} e^{-i q r}
$$

e

$$
\hat{J}(q)=\sum_{h} J(|h|) e^{-i q h}
$$

onde o vetor $\vec{h}$ corresponde à distância entre dois sítios no espaço direto. Fica evidente que $J\left(\vec{r}, \overrightarrow{r^{\prime}}\right)=J\left(\left|\vec{r}-\overrightarrow{r^{\prime}}\right|\right)$.

Sendo $S_{r}$ uma variável real, temos a relação $\hat{S}_{q}=\hat{S}_{-q}^{*}$. Podemos então lançar mão da transformação ortogonal,

$$
\left\{\begin{array} { r } 
{ \hat { S } _ { 0 } = \hat { R } _ { 0 } \quad q = 0 } \\
{ \hat { S } _ { q } = \frac { 1 } { \sqrt { 2 } } ( \hat { R } _ { q } + i \hat { I } _ { q } ) \quad \forall q \neq 0 }
\end{array} , \text { com } \quad \left\{\begin{array}{c}
\hat{R}_{q}=\hat{R}_{-q} \\
\hat{I}_{q}=-\hat{I}_{-q}
\end{array} .\right.\right.
$$


Usando essa transformação, a função de partição pode ser escrita como

$$
\begin{aligned}
Z_{G}(\beta) & =\int_{\mathcal{R}} \exp \left\{-\frac{1}{2}[b-\beta \hat{J}(0)] \hat{R}_{0}^{2}\right\} d \hat{R}_{0}\left[\prod_{q>0} \int_{\mathcal{R}} d \hat{R}_{q} d \hat{I}_{q} \exp \left\{-\frac{1}{2}(b-\beta \hat{J}(q))\left(\hat{R}_{q}^{2}+\hat{I}_{q}^{2}\right)\right\}\right] \\
& =\prod_{q}\left[\frac{2 \pi}{b-\beta \hat{J}(q)}\right]^{\frac{1}{2}} .
\end{aligned}
$$

É imediato notar que o resultado acima somente tem sentido para $b>\beta \hat{J}(q)$, qualquer que seja $q$ na primeira zona de Brillouin. Podemos então definir a temperatura crítica,

$$
\beta_{c}=\frac{b}{\max _{q}[\hat{J}(q)]} .
$$

Abaixo dessa temperatura a integral diverge e o modelo não tem nenhum sentido físico.

\subsection{Propriedades termodinâmicas}

A conexão com a termodinâmica é realizada através da energia livre (por spin) associada à função de partição canônica,

$$
\begin{aligned}
f(\beta) & =-\lim _{N^{d} \rightarrow+\infty} \frac{1}{N^{d} \beta} \ln Z_{G}(\beta) \\
& =-\frac{1}{2 \beta} \ln 2 \pi+\frac{1}{2 \beta} \lim _{N^{d \rightarrow+\infty}} \frac{1}{N^{d}} \sum_{q} \ln [b-\beta \hat{J}(q)] .
\end{aligned}
$$

Outras grandezas de interesse são a energia interna por spin $u(\beta)$, a entropia por spin $s(\beta)$ e o calor específico por spin $c(\beta)$. Para essas grandezas termodinâmicas obtemos os seguintes resultados:

$$
\begin{gathered}
u(\beta)=-\lim _{N^{d} \rightarrow+\infty} \frac{1}{N^{d}} \frac{\partial \ln Z_{G}(\beta)}{\partial \beta} \\
=-\frac{1}{2} \lim _{N^{d} \rightarrow+\infty} \frac{1}{N^{d}} \sum_{q} \frac{\hat{J}(q)}{b-\beta \hat{J}(q)}, \\
s(\beta)=k_{B} \beta[u(\beta)-f(\beta)] \\
=\lim _{N^{d} \rightarrow+\infty}\left\{\frac{k_{B} \ln 2}{2}-\frac{k_{B}}{2 N^{d}} \sum_{q} \ln [b-\beta \hat{J}(q)]-\frac{k_{B} \beta}{2 N^{d}} \sum_{q} \frac{\hat{J}(q)}{b-\beta \hat{J}(q)}\right\},
\end{gathered}
$$




$$
\begin{aligned}
c(\beta) & =-k_{B} \beta^{2} \frac{\partial u(\beta)}{\partial \beta} \\
& =\frac{k_{B} \beta^{2}}{2} \lim _{N^{d} \rightarrow+\infty} \frac{1}{N^{d}} \sum_{q} \frac{[\hat{J}(q)]^{2}}{[b-\beta \hat{J}(q)]^{2}} .
\end{aligned}
$$

Observa-se novamente que essas equações somente possuem algum significado físico acima da temperatura crítica.

Para encontrarmos a magnetização e a suscetibilidade por spin, devemos sujeitar o sistema a um campo magnético $H$ externo e uniforme (estamos interessados no limite de $H \rightarrow 0)$. Assim o novo hamiltoniano será dado por

$$
\mathcal{H}_{N^{d}}=-\frac{1}{2} \sum_{\vec{r}, \vec{r}^{\prime}} J_{\vec{r}, \vec{r}^{\prime}} S_{\vec{r}} S_{\vec{r}^{\prime}}-H \sum_{\vec{r}} S_{\vec{r}}
$$

Os cálculos para obter a nova função de partição são análogos. A magnetização e a suscetibilidade por spin a campo nulo são dadas por

$$
m(\beta, H \rightarrow 0)=-\left(\frac{\partial f}{\partial H}\right)_{\beta, H=0}=\frac{\beta H}{b-\beta \hat{J}(0)}
$$

e

$$
\mathcal{X}(\beta, H \rightarrow 0)=\left.\frac{\partial m}{\partial H}\right|_{H=0}=\frac{\beta}{b-\beta \hat{J}(0)}
$$

\subsection{Modelo ferromagnético com interações de primeiros vizi- nhos}

Agora vamos particularizar e aprofundar nossa análise na rede hipercúbica para interações restritas a primeiro vizinhos. Já que a interação de troca só depende da distância entre os sítios, $J(\vec{h})=J(-\vec{h})$. Tomando um parâmetro de rede unitário, temos

$$
\hat{J}(q)=2 J \sum_{i=1}^{d} \cos q_{i} .
$$

Considerando essa relação, é evidente que para interações ferromagnéticas $(J>0)$ o maior valor de $\hat{J}(q)$, que determina a temperatura crítica, ocorre para $q$ nulo $[\vec{q}=$ $\left.\left(q_{1}, q_{2}, \cdots, q_{d}\right)\right]$. Logo, 


$$
\beta_{c}=\frac{b}{2 J d},
$$

em que $J$ é a interação de troca entre primeiros vizinhos e $d$ é a dimensionalidade da rede.

As grandezas termodinâmicas encontradas precedentemente podem ser reescritas considerando a expressão (2.20). Doravante vamos reescrevê-las para $T \rightarrow T_{c}$, e por conseqüência $q \rightarrow q_{c}=0$.

Levando em conta o limite termodinâmico temos a correspondência

$$
\frac{1}{N^{d}} \sum_{\forall q \neq 0}=\frac{1}{(2 \pi)^{d}} \int d^{d} q,
$$

onde a integração deverá ser feita no hipercubo que define a primeira zona de Brillouin.

Como já foi dito no penúltimo parágrafo, o comportamento singular das funções termodinâmicas se faz para comprimentos de onda pequenos. Isso justifica a expansão

$$
\cos q_{i}=1-\frac{q_{i}^{2}}{2}+\mathcal{O}\left(q_{i}^{4}\right),
$$

com

$$
q_{1}^{2}+q_{2}^{2}+\cdots+q_{d}^{2}=q^{2} .
$$

Uma vez que vamos usar coordenadas esféricas, somente a parte radial é que apresenta singularidade. A menos de um fator angular, o comportamento singular da integral (2.22) é representado por

$$
\frac{1}{(2 \pi)^{d}} \int d^{d} q \longrightarrow \frac{1}{(2 \pi)^{d}} \int_{\epsilon}^{a} q^{d-1} d q,
$$

onde $\epsilon$ é o raio infinitesimal de uma hiperesfera centrada na origem (ponto singular) e dentro da hiperesfera de raio $a$. Assim, quando escrevermos as grandezas termodinâmicas que seguem, nas imediatas vizinhanças de $q=0$, estaremos analisando somente a parte radial.

\section{Energia livre por spin}

Substituindo (2.22) em (2.13), vem

$$
\begin{aligned}
f(\beta) & =-\frac{1}{2 \beta} \ln 2 \pi+\frac{1}{2 \beta(2 \pi)^{d}} \int d^{d} q \ln [b-\beta \hat{J}(q)] \\
& \sim-\frac{1}{2 \beta_{c}} \ln 2 \pi+\frac{1}{2 \beta_{c}(2 \pi)^{d}} \int_{\epsilon=0}^{a} q^{d-1} \ln \left(\frac{b q^{2}}{2 d}\right) d q
\end{aligned}
$$


onde usamos a equação (2.12) e a expansão (2.23). Fazendo uma integração por partes, é fácil perceber que a expressão acima converge para todo $d \geq 1$.

\section{Energia interna por spin}

Substituindo (2.22) em (2.14), temos

$$
\begin{aligned}
u(\beta) & =-\frac{1}{2(2 \pi)^{d}} \int d^{d} q \frac{\hat{J}(q)}{b-\beta \hat{J}(q)} \\
& \sim-\frac{d}{\beta_{c}(2 \pi)^{d}} \int_{\epsilon=0}^{a} \frac{q^{d-1}}{2 d t+q^{2}+\mathcal{O}\left(q^{4}\right)} d q
\end{aligned}
$$

onde introduzimos a temperatura reduzida $t=\left(T-T_{c}\right) / T_{c}$ e fizemos uma expansão para $t$ indo a zero. É claro que a expressão acima é formada pelos termos dominantes das expansões.

A integral na relação da energia interna por spin poderá apresentar problemas nas proximidades de seu limite inferior $(t \rightarrow 0)$; nas proximidades do limite superior, porém, como a está dentro da primeira zona de Brillouin, não temos problemas, ou seja, a integral sempre converge nesse extremo.

O comportamento crítico pode ser investigado a partir da integral no plano complexo

$$
I=\int_{\mathcal{C}} \frac{z^{d-1} e^{-\alpha z z^{*}} \ln z}{z^{2}+s} d z,
$$

com $\alpha>0$ e $\mathcal{C}$, dado por $\mathrm{O}$ ponto de ramificação surge pois o logaritmo é uma função

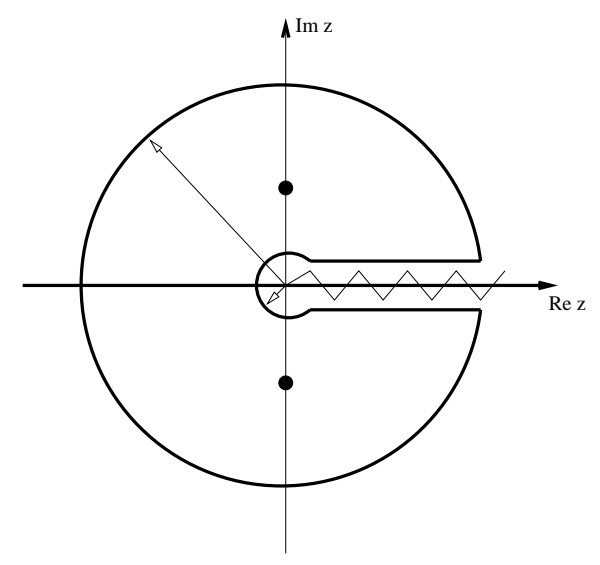

Figura 2.1: Contorno no plano complexo

plurívoca. Ainda, $\ln z=\ln q$ na trajetória $\operatorname{logo}$ acima do eixo real e $\ln z=\ln q+2 \pi i$ na trajetória logo abaixo. 
É possivel mostrar que as integrações sobre o círculo maior e sobre o círculo menor serão nulas, quando os raios são muito grande e muito pequeno, respectivamente. Então, o teorema dos resíduos nos garante que

$$
\begin{aligned}
I & =\int_{0}^{\infty} \frac{q^{d-1} e^{-\alpha q^{2}} \ln q}{q^{2}+s} d q+\int_{\infty}^{0} \frac{q^{d-1} e^{-\alpha q^{2}}(\ln q+2 \pi i)}{q^{2}+s} d q=2 \pi i \text { Lresíduos } \\
& =-\int_{0}^{\infty} \frac{q^{d-1} e^{-\alpha q^{2}}}{q^{2}+s} d q=\text { Lresíduos. }
\end{aligned}
$$

Após alguns cálculos, pode ser mostrado que a integral tem a forma

$$
\int_{0}^{\infty} \frac{q^{d-1} e^{-\alpha q^{2}}}{q^{2}+s} d q=A(d) e^{\alpha s} s^{\frac{d-2}{2}}
$$

Fazendo $\alpha=0$, temos o mesmo integrando presente na expressão da energia interna, com $s=2 d t$. É claro, então, que, a energia interna irá convergir para todo $d \geq 2$. O caso unidimensional será tratado em detalhe em breve.

\section{Entropia por spin}

De $(2.15)$ e $(2.22)$, temos

$$
s(\beta)=\frac{k_{B} \ln 2}{2}-\frac{k_{B}}{2(2 \pi)^{d}} \int d^{d} q \ln [b-\beta \hat{J}(q)]-\frac{k_{B} \beta}{2(2 \pi)^{d}} \int d^{d} q \frac{\hat{J}(q)}{b-\beta \hat{J}(q)} .
$$

Os mesmos argumentos valem para a entropia e mostram que essa só apresentará problemas para $d=1$.

\section{Calor específico}

De (2.16) e (2.22), temos

$$
\begin{aligned}
c(\beta) & =\frac{k_{B} \beta^{2}}{2(2 \pi)^{d}} \int d^{d} q \frac{[\hat{J}(q)]^{2}}{[b-\beta \hat{J}(q)]^{2}} \\
& \sim \frac{2 k_{B} d^{2}}{(2 \pi)^{d}} \int_{\epsilon=0}^{a} \frac{q^{d-1}}{\left[2 d t+q^{2}\right]^{2}} d q .
\end{aligned}
$$

Outra vez, pelos mesmos argumentos apresentados no estudo do comportamento crítico da energia interna, poderemos ter anomalias nas vizinhanças da temperatura crítica, e não há divergêcia no limite superior da integral. 
Agora a integral complexa que deve ser calculada tem a forma

$$
I=\int_{\mathcal{C}} \frac{z^{d-1} e^{-\alpha z z^{*}} \ln z}{\left(z^{2}+s\right)^{2}} d z
$$

com o mesmo contorno usado no caso da energia interna.

Praticamente os mesmos cálculos da energia interna são reproduzidos aqui, porém, temos pólos de segunda ordem. Assim, podemos escrever que nas proximidades da temperatura crítica o calor específico toma a forma

$$
c(t) \sim K(d)(2 d t)^{\frac{d-4}{2}}
$$

Essa relação fornece o expoente crítico $\alpha$, que é dependente da dimensão. Para $d<4$, fica evidente que o calor específico diverge na temperatura crítica. Para $d \geqslant 4$, não há problemas e costuma-se fazer $\alpha=0(d \geqslant 4)$ remetendo ao valor de $\alpha$ da aproximação de campo médio.

\section{Magnetização espontânea}

Como não há significado físico em um modelo gaussiano abaixo da temperatura crítica, não estamos aptos a definir o expoente crítico $\beta$.

\section{Suscetibilidade magnética a campo nulo}

Podemos escrever a equação (2.19) como

$$
\mathcal{X}(t, h \rightarrow 0)=\frac{\beta_{c}}{b(t+1)-2 J d}
$$

Assim

$$
\mathcal{X}(t, H \rightarrow 0) \sim \frac{\beta_{c}}{b} t^{-1}
$$

de onde vem o expoente crítico $\gamma=1$.

\subsection{Análise da energia interna por spin para $d=1$}

É instrutivo encontrar uma expressão exata para a energia interna por spin do modelo unidimensional, com interações ferromagnéticas entre primeiros vizinhos, e demonstrar 
explicitamente sua divergência na temperatura crítica.

Para $d=1$ temos

$$
u(\beta)=-\frac{1}{4 \pi} \int_{-\pi}^{\pi} d q \frac{2 J \cos q}{b-2 J \beta \cos q},
$$

$\operatorname{com} b=2 J \beta_{c}$.

Assim,

$$
u(\beta)=-\frac{1}{4 \pi \beta_{c}} \int_{-\pi}^{\pi} d q \frac{\cos q}{1-\theta \cos q}
$$

onde $\theta=\beta / \beta_{c}=T_{c} / T$.

A integral acima pode ser convertida em uma integral de contorno no plano complexo através da transformação

$$
\cos q=\frac{1}{2}\left(z+\frac{1}{z}\right)
$$

$\operatorname{com} z=e^{i q}$. Uma vez efetuada essa transformação, temos

$$
u(\beta)=\frac{1}{4 \pi \beta_{c}} \int_{C} \frac{d z}{i z} \frac{\frac{1}{2}\left(z+\frac{1}{z}\right)}{1-\frac{\theta}{2}\left(z+\frac{1}{z}\right)},
$$

sendo $C$ o círculo unitário centrado na origem do plano complexo. Fazendo algumas manipulações algébricas podemos escrever

$$
\begin{aligned}
u(\beta) & =\frac{1}{4 \pi \beta} \int_{C} d z \frac{z^{2}+1}{i z\left(z^{2}-\frac{2}{\theta} z+1\right)} \\
& =\frac{1}{4 \pi \beta} \int_{C} d z \frac{z^{2}+1}{i z\left(z-z_{+}\right)\left(z-z_{-}\right)}
\end{aligned}
$$

onde as raízes $z_{+}$e $z_{-}$são

$$
z_{ \pm}=\frac{1}{\theta}\left(1 \pm \sqrt{1-\theta^{2}}\right)
$$

A penúltima expressão pode ser calculada com o auxílio do método dos resíduos. Há pólos em $z=0, z=z_{+}$e em $z=z_{-}$, mas apenas os pólos que estiverem dentro do circulo unitário contribuirão para o cálculo de $u(\beta)$. Além de $z=0$, os dois outros pólos podem ou não estar dentro do círculo unitário, necessitando assim uma verificação.

- O pólo $z=z_{+}$é dado por

$$
z_{+}=\frac{1}{\theta}\left(1+\sqrt{1-\theta^{2}}\right)=\frac{T}{T_{c}}\left(1+\sqrt{1-\frac{T_{c}^{2}}{T^{2}}}\right) .
$$


Levando em conta que $T>T_{c}$, pois o modelo não é definido abaixo da temperatura crítica, temos

$$
\left|z_{+}\right|>1
$$

Portanto, esse pólo não contribuirá para a energia interna.

- O pólo $z=z_{-}$é dado por

$$
z_{-}=\frac{1}{\theta}\left(1-\sqrt{1-\theta^{2}}\right)=\frac{T}{T_{c}}\left(1-\sqrt{1-\frac{T_{c}^{2}}{T^{2}}}\right) .
$$

Não é trivial verificar se o pólo $z_{-}$está ou não dentro do contorno. Devemos analisar para quais valores de temperatura ele estará dentro do contorno, contribuindo assim à energia interna. Vamos supor que

$$
\left|z_{-}\right|<1 \rightarrow \frac{T}{T_{c}}\left(1-\sqrt{1-\frac{T_{c}^{2}}{T^{2}}}\right)<1
$$

ou seja,

$$
\left(\frac{T_{c}^{2}}{T^{2}}\right)^{2}-\frac{T_{c}}{T}<0
$$

A última relação será satisfeita para todo $T_{c}<T<\infty$, isto é, para toda região de temperaturas em que o modelo é definido. Assim, $z_{-}$está sempre dentro do circulo unitário e a expressão da energia pode ser escrita como

$$
\begin{aligned}
u(\beta) & =\frac{2 \pi i}{4 \pi \beta}\left[\operatorname{Res}(z=0)+\operatorname{Res}\left(z=z_{-}\right)\right] \\
& =\frac{i}{2 \beta}\left[\lim _{z \rightarrow 0}\left(z \frac{z^{2}+1}{i z\left(z-z_{+}\right)\left(z-z_{-}\right)}\right)+\lim _{z \rightarrow z_{-}}\left(\left(z-z_{-}\right) \frac{z^{2}+1}{i z\left(z-z_{+}\right)\left(z-z_{-}\right)}\right)\right](2 .
\end{aligned}
$$

Substituindo as expressões associadas às raízes e após alguns passos algébricos, obtemos

$$
u(\beta)=-\frac{\beta}{\beta_{c}^{2}-\beta^{2}+\beta_{c} \sqrt{\beta_{c}^{2}-\beta}} .
$$

A partir dessa relação verificamos que a energia interna para $d=1$ diverge para $T \rightarrow$ $T_{c}$ com $t^{-1 / 2}$, sendo $t$ a temperatura reduzida. Isso mostra a artificialidade do modelo gaussiano unidimensional, pois não há nenhuma característica termodinâmica confiável em um modelo cuja energia interna por spin diverge na temperatura crítica! 


\subsection{Função de correlação de pares de spins}

As funções de correlação são grandezas de importância singular em mecânica estatística, pois carregam consigo importantes informações, sobretudo para um contexto de análise crítica de um modelo. Elas são calculadas através da relação,

$$
\Gamma_{G}\left(\vec{r}, \vec{r}^{\prime}\right)=\left\langle\left(S_{\vec{r}}-\left\langle S_{\vec{r}}\right\rangle\right)\left(S_{\vec{r}^{\prime}}-\left\langle S_{\vec{r}^{\prime}}\right\rangle\right)\right\rangle
$$

Considerando um sistema sem campo magnético, e levando em conta sua invariância por translação, podemos escrever

$$
\Gamma_{G}(0, r)=\left\langle S_{0} S_{r}\right\rangle=\frac{1}{N^{d}} \sum_{q, q^{\prime}} e^{-i q^{\prime} r}\left\langle\hat{S}_{q} \hat{S}_{q^{\prime}}\right\rangle,
$$

onde usamos a transformação (2.6). Devemos lembrar agora que, no espaço recíproco, $\hat{S}_{q}$ se acopla somente com $\hat{S}_{-q}$. Logo,

$$
\Gamma_{G}(0, r)=\frac{1}{2 N^{d}} \sum_{q} e^{-i q r}\left\langle\hat{S}_{q} \hat{S}_{-q}\right\rangle .
$$

Usando a transformação ortogonal (2.10), temos

$$
\begin{aligned}
\Gamma_{G}(0, r) & =\frac{1}{2 N^{d}} \sum_{q} e^{-i q r}\left\langle\left(\hat{R}_{q}^{2}+\hat{I}_{q}^{2}\right)\right\rangle \\
& =\frac{1}{N^{d}} \sum_{q} \frac{e^{-i q r}}{b-\beta \hat{J}(q)} .
\end{aligned}
$$

\section{Caso ferromagnético com interações de primeiros vizinhos}

Vamos agora analisar o comportamento da função de correlação na temperatura crítica e nas suas imediatas vizinhanças para um modelo ferromagnético de primeiros vizinhos, definido sobre uma rede hipercúbica, com parâmetro de rede igual à unidade.

Através do mesmo procedimento aplicado às funções termodinâmicas, usando as equações $(2.52)$ e $(2.22)$, temos

$$
\Gamma(0, r)=\frac{1}{(2 \pi)^{d}} \int d^{d} q \frac{e^{-i q r}}{b-\beta \hat{J}(q)} .
$$


Para o caso de estarmos na temperatura crítica, o termo dominante é dado por

$$
\Gamma(0, r) \sim \frac{1}{(2 \pi)^{d} \beta_{c} J} \int_{\epsilon=0}^{a} e^{-i q r} q^{d-3} d q .
$$

Vamos agora introduzir a mudança de variáveis

$$
x=q r .
$$

Então, temos a forma das correlações de pares a longas distâncias,

$$
\Gamma(0, r) \sim\left[\frac{1}{J \beta_{c}(2 \pi)^{d}} \int_{\epsilon=0}^{\infty} d x x^{d-3} e^{-i x}\right] \frac{1}{r^{d-2}} .
$$

Sabendo que no ponto crítico, para $r \rightarrow \infty$, as correlações se comportam como

$$
\Gamma(0, r) \sim r^{-(d-2+\eta)}
$$

é claro que $\eta=0$.

Nas proximidades da temperatura crítica, de maneira análoga às expansões realizadas anteriormente, podemos escrever

$$
\Gamma(0, r) \sim \frac{1}{J \beta_{c}(2 \pi)^{d}} \int_{\epsilon=0}^{a} \frac{q^{d-1} e^{-i q r}}{q^{2}+2 d t} d q
$$

que irá divergir na temperatura crítica apenas para um modelo unidimensional. Sendo assim o caso unidimensional a situação interasante a ser considerada. Consideremos a integral complexa

$$
I=\int_{\mathcal{C}} d z \frac{e^{-i z r}}{z^{2}+2 d t}
$$

que possui integrando par e onde o contorno $\mathcal{C}$ é um semicirculo no plano inferior complexo. Novamente pode mostrar-se que a integração sobre o semicirculo inferior com raio muito grande é nula e com o auxílio do teorema dos resíduos vemos que a função de correlação de pares fica

$$
\Gamma^{d=1}(0, r) \sim-\frac{\pi(2 d t)^{-\frac{1}{2}}}{J \beta_{c}(2 \pi)^{d}} \exp \left[-\frac{r}{(2 d t)^{-\frac{1}{2}}}\right],
$$

que fornece o expoente crítico associado ao comprimento de correlação, $\nu=1 / 2$.

Os expoentes críticos $\eta=0$ e $\nu=1 / 2$ são as características marcantes do modelo gaussiano (e das teorias de campo médio). Esses resultados, ainda, estão em sintonia com a teoria clássica de Ornstein-Zernicke, sobre o decaimento das funções de dois pontos (correlações) nas vizinhanças da criticalidade. 


\section{Capítulo 3}

\section{Comportamento dinâmico}

Como já foi dito, o modelo gaussiano é um modelo definido no equilíbrio. Devemos então adicionar uma dinâmica a esse sistema. Nesse contexto vamos analisar um comportamento dinâmico do modelo gaussiano segundo dois tipos de dinâmica: a dinâmica dissipativa do tipo $A$ e a dinâmica do tipo $B$.

Esses dois tipos de dinâmica serão investigados nas proximidades do ponto crítico do modelo gaussiano. O que se procurará encontrar serão expressões para a função de autocorrelação, que se refere à correlação de um mesmo sítio em instantes diferentes, e a função resposta a um estímulo externo pequeno.

Em um estágio mais avançado usaremos uma outra técnica para estudar o comportamento dinâmico do modelo gaussiano, conhecida com formalismo MSR. Essa técnica foi desenvolvida num contexto de teorias de campos e adaptada ao estudo de comportamentos dinâmicos em sistemas com interações de spins, sobretudo para a investigação de sistemas com desordem. Finalmente estudaremos o comportamento dinâmico do modelo gaussiano na presença de um campo aleatório.

\subsection{Modelo gaussiano crítico na dinâmica de Langevin}

A dinâmica de Langevin nasceu com o estudo do movimento browniano. As idéias do atomismo explicaram, de forma aproximada, mas extremamente eficaz, o movimento errático de uma partícula em um fluido, através de colisões entre as partículas, muito menores, constituintes do fluido com a partícula ali depositada. Nesse contexto de mo- 
vimento browniano, a dinâmica de Langevin considera que além de uma força viscosa também atua sobre a partícula browniana uma força de carater aleatório (ruído) devido aos impactos com todas as partículas menores do fluido. No tocante ao ruído, considera-se usualmente uma distribuição gaussiana, com média nula.

De uma forma generalizada a dinâmica de Langevin para as variáveis de spin no espaço real é representada pela equação

$$
\frac{\partial S_{r}}{\partial t}=-\frac{\delta}{\delta S_{r}}\left[\mathcal{H}^{\prime}\left\{S_{r}(t)\right\}\right]+\eta_{r}(t)
$$

onde usamos o hamiltoniano efetivo do modelo gaussiano,

$$
\mathcal{H}^{\prime}\left\{S_{r}(t)\right\}=-\frac{1}{2} \sum_{r, r^{\prime}} J_{r, r^{\prime}} S_{r} S_{r^{\prime}}+\frac{b}{2 \beta} \sum_{r} S_{r}^{2},
$$

e um ruído descorrelacionado, que obedece as relações

$$
\left\langle\eta_{r}(t)\right\rangle=0 \quad e \quad\left\langle\eta_{r}(t) \eta_{r^{\prime}}\left(t^{\prime}\right)\right\rangle=\frac{2}{\beta} \delta_{r, r^{\prime}} \delta\left(t-t^{\prime}\right)
$$

Uma vez que

$$
\frac{\delta}{\delta S_{r}}\left[\mathcal{H}^{\prime}\left\{S_{r}(t)\right\}\right]=-\sum_{r^{\prime}} J_{r, r^{\prime}} S_{r^{\prime}}+\frac{b}{\beta} S_{r},
$$

a equação de Langevin pode ser escrita na forma

$$
\frac{\partial S_{r}(t)}{\partial t}=\sum_{r^{\prime}} J_{r, r^{\prime}} S_{r^{\prime}}-\frac{b}{\beta} S_{r}+\eta_{r}(t) .
$$

Devemos aqui relembrar que para não carregar a notação não estamos explicitando o caráter vetorial das variáveis.

Visando eliminar acoplamentos, a transformada discreta de Fourier é aplicada à última relação obtendo-se,

$$
\frac{\partial \hat{S}_{q}(t)}{\partial t}=\left[\hat{J}(q)-\frac{b}{\beta}\right] \hat{S}_{q}(t)+\hat{\eta}_{q}(t)
$$

onde

$$
\begin{aligned}
\hat{S}_{q}(t) & =\frac{1}{N^{\frac{d}{2}}} \sum_{r} e^{-i q r} S_{r}(t), \\
\hat{J}(q) & =\sum_{h} J(h) e^{-i q h}, \\
\hat{\eta}_{q}(t) & =\frac{1}{N^{\frac{d}{2}}} \sum_{r} e^{-i q r} \eta_{q}(t) .
\end{aligned}
$$


A equação (3.5) é uma equação diferencial linear de primeira ordem e não homogênea, cuja solução é dada por

$$
\hat{S}_{q}(t)=\hat{S}_{q}(0) \exp \left\{\left[\hat{J}(q)-\frac{b}{\beta}\right] t\right\}+\exp \left\{\left[\hat{J}(q)-\frac{b}{\beta}\right] t\right\} \int_{0}^{t} \exp \left\{-\left[\hat{J}(q)-\frac{b}{\beta}\right] t^{\prime}\right\} \hat{\eta}_{q}\left(t^{\prime}\right) d t^{\prime}
$$

que evidentemente depende da condição inicial.

O nosso objetivo é calcular a função de correlação e a função resposta para o modelo gaussiano, que são os observáveis de interesse, além de verificar a validade do teorema de flutuação-dissipação nesse enfoque dinâmico. Para tanto vamos escrever

$$
\left\langle S_{r}(t) S_{r^{\prime}}\left(t^{\prime}\right)\right\rangle=\frac{1}{N^{d}} \sum_{q, q^{\prime}} e^{i q r+i q^{\prime} r^{\prime}}\left\langle\hat{S}_{q}(t) \hat{S}_{q^{\prime}}\left(t^{\prime}\right)\right\rangle
$$

onde foi usada a relação (2.6). Para o mesmo valor de $r$, temos a auto-correlação

$$
\left\langle S_{r}(t) S_{r}\left(t^{\prime}\right)\right\rangle=\frac{1}{N^{d}} \sum_{q, q^{\prime}} e^{i\left(q+q^{\prime}\right) r}\left\langle\hat{S}_{q}(t) \hat{S}_{q^{\prime}}\left(t^{\prime}\right)\right\rangle .
$$

Multiplicando a expressão acima por $N^{-d}$ e somando sobre todas as posições, temos

$$
\frac{1}{N^{d}} \sum_{r}\left\langle S_{r}(t) S_{r}\left(t^{\prime}\right)\right\rangle=\frac{1}{N^{d}} \sum_{q \cdot q^{\prime}}\left\langle\hat{S}_{q}(t) \hat{S}_{q^{\prime}}\left(t^{\prime}\right)\right\rangle \frac{1}{N^{d}} \sum_{r} e^{i\left(q+q^{\prime}\right) r}
$$

Identificando os termos dependentes de $r$ do lado direito da última relação com o delta de Kronecker $\delta_{q,-q^{\prime}}$, temos a definição da auto-correlação

$$
C\left(t, t^{\prime}\right) \equiv \frac{1}{N^{d}} \sum_{r}\left\langle S_{r}(t) S_{r}\left(t^{\prime}\right)\right\rangle=\frac{1}{N^{d}} \sum_{q}\left\langle\hat{S}_{q}(t) \hat{S}_{-q}\left(t^{\prime}\right)\right\rangle .
$$

Salienta-se, mais uma vez que, o acoplamento no espaço recíproco se dá somente entre $q$ e $-q$. Nesse sentido, aplicando a transformada de Fourier à equação (3.3), temos

$$
\left\langle\hat{\eta}_{q}(t) \hat{\eta}_{q^{\prime}}\left(t^{\prime}\right)\right\rangle=\frac{2}{\beta} \delta_{q,-q^{\prime}} \delta\left(t-t^{\prime}\right)
$$

Para calcular a auto-correlação, usamos a equação (3.8), assim

$$
\begin{aligned}
C\left(t, t^{\prime}\right)=\frac{1}{N^{d}} \sum_{q}\langle & {\left[\hat{S}_{q}(0) e^{-\omega(q) t}+\int_{0}^{t} e^{-\omega(q)\left(t-t_{1}\right)} \hat{\eta}_{q}\left(t_{1}\right) d t_{1}\right] \times } \\
& {\left.\left[\hat{S}_{-q}(0) e^{-\omega(-q) t^{\prime}}+\int_{0}^{t^{\prime}} e^{-\omega(-q)\left(t^{\prime}-t_{2}\right)} \hat{\eta}_{-q}\left(t_{2}\right) d t_{2}\right]\right\rangle, }
\end{aligned}
$$


onde

$$
\omega(q)=\frac{b}{\beta}-\hat{J}(q)
$$

Uma vez que a interação de troca só depende da distância entre os sítios, também temos $\hat{J}(q)=\hat{J}(-q)$. Podemos então escrever

$$
\begin{aligned}
C\left(t, t^{\prime}\right) & =\frac{1}{N^{d}} \sum_{q}\left\{e^{-\omega(q)\left(t+t^{\prime}\right)}\left\langle\hat{S}_{q}(0) \hat{S}_{-q}(0)\right\rangle+e^{-\omega(q)\left(t+t^{\prime}\right)} \int_{0}^{t} d t_{1} \int_{0}^{t^{\prime}} d t_{2} e^{\omega(q)\left(t_{1}+t_{2}\right)} \frac{2}{\beta} \delta\left(t_{1}-t_{2}\right)\right\} \\
& =\frac{1}{N^{d}} \sum_{q}\left\{e^{-\omega(q)\left(t+t^{\prime}\right)}\left\langle\hat{S}_{q}(0) \hat{S}_{-q}(0)\right\rangle+\frac{2}{\beta} e^{-\omega(q)\left(t+t^{\prime}\right)} \int_{0}^{t} d t_{1} e^{2 \omega(q) t_{1}} \theta\left(t^{\prime}-t_{1}\right)\right\},
\end{aligned}
$$

onde estamos considerando $t>t^{\prime}$; ainda introduzindo a função de Heaviside $\theta(t)$ (cálculos desse tipo podem ser encontrados em (Salinas, 1997), no capítulo 16, e em (Zwanzig, 2000), capítulo 1). Finalmente temos

$$
C\left(t, t^{\prime}\right)=\frac{1}{N^{d}} \sum_{q}\left\{e^{-\omega(q)\left(t+t^{\prime}\right)}\left\langle\hat{S}_{q}(0) \hat{S}_{-q}(0)\right\rangle+\frac{1}{\beta \omega(q)}\left[e^{-\omega(q)\left(t-t^{\prime}\right)}-e^{-\omega(q)\left(t+t^{\prime}\right)}\right]\right\} .
$$

Note que a auto-correlação também depende das condições iniciais. Supõe-se, daqui por diante, um sistema que em $t=0$ possua uma temperatura efetivamente infinita (spins totalmente descorrelacionados) e sofre então um processo de resfriamento ("quenching") (Hase, 2005). É importante dizer que essa escolha não é obrigatória, podendo ter sido feitas outras. O argumento de que o sistema possui inicialmente uma temperatura efetivamente infinita foi feito para adotar um mesmo comportamento inicial médio para todos os sítios do sistema, trazendo simplificações aos cálculos. Usando a equação (2.6), temos

$$
\left\langle\hat{S}_{q}(0) \hat{S}_{-q}(0)\right\rangle=C_{q}(0,0)=\frac{1}{N^{d}} \sum_{r, r^{\prime}}\left\langle S_{r}(0) S_{r^{\prime}}(0)\right\rangle e^{-i\left(r-r^{\prime}\right) q}
$$

Levando em conta as condições iniciais, escrevemos

$$
\left\langle S_{r}(0) S_{r^{\prime}}(0)\right\rangle=\left\{\begin{array}{rr}
\left\langle S^{2}(0)\right\rangle, & r=r^{\prime} \\
\left\langle S_{r}(0)\right\rangle\left\langle S_{r^{\prime}}(0)\right\rangle=0, & r \neq r^{\prime}
\end{array} .\right.
$$

Este resultado, quando aplicado à equação (3.18), fornece

$$
C_{q}(0,0)=\left\langle S^{2}(0)\right\rangle
$$

Para analisar a função resposta do modelo gaussiano, adicionamos um termo de campo magnético auxiliar $H_{r}(t)$, e realizamos os cálculos no limite de campo nulo. Assim, o novo 
hamiltoniano efetivo do sistema (correspondente a uma energia livre), é dado por

$$
\mathcal{H}^{\prime}\left\{S_{r}(t)\right\}=-\frac{1}{2} \sum_{r, r^{\prime}} J_{r, r^{\prime}} S_{r} S_{r^{\prime}}-\sum_{r} H_{r} S_{r}+\frac{b}{2 \beta} \sum_{r} S_{r}^{2} .
$$

A equação de Langevin é agora escrita como

$$
\frac{\partial S_{r}(t)}{\partial t}=\sum_{r^{\prime}} J_{r, r^{\prime}} S_{r^{\prime}}(t)-\frac{b}{\beta} S_{r}(t)+H_{r}(t)+\eta_{r}(t) .
$$

Apelando novamente à transformada de Fourier, encontramos

$$
\frac{\partial \hat{S}_{q}(t)}{\partial t}=\left[\hat{J}(q)-\frac{b}{\beta}\right] \hat{S}_{q}(t)+\hat{H}_{q}(t)+\hat{\eta}_{q}(t)
$$

onde

$$
\hat{H}_{q}(t)=\frac{1}{N^{\frac{d}{2}}} \sum_{r} H_{r}(t) e^{-i q r}
$$

com as demais grandezas dadas pela equação (3.7). A solução da equação (3.23) é escrita como

$$
\hat{S}_{q}(t)=\hat{S}_{q}(0) e^{-\omega(q) t}+\int_{0}^{t} e^{-\omega(q)\left(t-t_{1}\right)}\left[\hat{H}_{q}\left(t_{1}\right)+\hat{\eta}_{q}\left(t_{1}\right)\right] d t_{1}
$$

com $\omega(q)$ dado pela definição (3.15).

Passemos agora a considerar o valor médio de $\hat{S}_{q}(t)$,

$$
\left\langle\hat{S}_{q}(t)\right\rangle=\int_{0}^{t} e^{-\omega(q)\left(t-t_{1}\right)} \hat{H}_{q}\left(t_{1}\right) d t_{1} .
$$

A função resposta do sistema é definida como

$$
R\left(t, t^{\prime}\right)=\left.\frac{1}{N^{d}} \sum_{r} \frac{\delta\left\langle S_{r}(t)\right\rangle}{\delta H_{r}\left(t^{\prime}\right)}\right|_{H=0}=\left.\frac{1}{N^{d}} \sum_{q} \frac{\delta\left\langle\hat{S}_{q}(t)\right\rangle}{\delta \hat{H}_{q}\left(t^{\prime}\right)}\right|_{H=0},
$$

em que a derivada funcional de $F$ em relação a $f$ no ponto $x$ é definida implicitamente por

$$
\left(\frac{d}{d \epsilon} F[f+\epsilon \sigma]\right)_{\epsilon=0} \equiv \int_{\mathcal{R}^{n}} d^{n} x \sigma(x) \frac{\delta F}{\delta f(x)} .
$$

Fazendo $\left\langle\hat{S}_{q}(t)\right\rangle \equiv\left\langle\hat{S}_{q}\left(t,\left[\hat{H}_{q}(t)\right]\right)\right\rangle$, temos

$$
\begin{aligned}
\left\langle\hat{S}_{q}\left(t,\left[\hat{H}_{q}(t)+\epsilon \sigma_{q}(t)\right]\right)\right\rangle & =\int_{0}^{t} d t_{1} e^{-\omega(q)\left(t-t_{1}\right)}\left[\hat{H}_{q}\left(t_{1}\right)+\epsilon \sigma_{q}\left(t_{1}\right)\right] d t_{1} \\
& =\left\langle\hat{S}_{q}\left(t,\left[\hat{H}_{q}(t)\right]\right)\right\rangle+\epsilon \int_{0}^{t} e^{-\omega(q)\left(t-t_{1}\right)} \sigma_{q}\left(t_{1}\right) d t_{1}
\end{aligned}
$$

Para $\epsilon \rightarrow 0$, usando as equações (3.29), (3.28) e (3.27), temos 


$$
R\left(t, t^{\prime}\right)=\frac{1}{N^{d}} \sum_{q} e^{-\omega(q)\left(t-t^{\prime}\right)} .
$$

Vamos agora verificar se o teorema de flutuação-dissipação (TFD) é satisfeito nas proximidades da temperatura crítica do modelo gaussiano dinâmico. Esse teorema relaciona o mecanismo de dissipação de energia com as flutuações térmicas do equilíbrio (Zwanzig, 2000) (Crisanti e Ritort, 2003) (vide apêndice A). Ele é sempre válido para o equilíbrio e possui muitas formulações. No entanto, pode ser violado para sistemas fora do equilíbrio (Crisanti e Ritort, 2003). O TFD estabelece a conexão entre a função resposta e a função de correlação,

$$
\frac{\partial C\left(t, t^{\prime}\right)}{\partial t^{\prime}}=\frac{R\left(t, t^{\prime}\right)}{\beta} .
$$

Como essa relação pode ser alterada em situações fora do equilíbrio, vamos calcular a razão

$$
X=\frac{R\left(t, t^{\prime}\right)}{\beta \frac{\partial C\left(t, t^{\prime}\right)}{\partial t^{\prime}}},
$$

que tem sido estudada por diversos autores.

Nas condições de validade do teorema de flutuação-dissipação, $X=1$. A versão modificada do TFD tem sido interpretada como indicadora de uma temperatura efetiva do sistema, dada por $\beta_{e f}=X \beta$ (Crisanti e Ritort, 2003) (Cugliandolo, 2002).

Assim, das equações (3.17), (3.20), (3.30) e (3.32), temos

$$
\begin{aligned}
X & =\frac{\frac{1}{N^{d}} \sum_{q} \exp \left[-\omega(q)\left(t-t^{\prime}\right)\right]}{\frac{\beta}{N^{d}} \frac{\partial}{\partial t^{\prime}}\left\{\sum_{q}\left[e^{-\omega(q)\left(t+t^{\prime}\right)}\left\langle S^{2}(0)\right\rangle+\frac{1}{\beta \omega(q)}\left[e^{-\omega(q)\left(t-t^{\prime}\right)}-e^{-\omega(q)\left(t+t^{\prime}\right)}\right]\right]\right\}} \\
& =\left\{-\frac{\beta \sum_{q} \omega(q) e^{-\omega(q)\left(t+t^{\prime}\right)}\left\langle S^{2}(0)\right\rangle}{\sum_{q} e^{-\omega(q)\left(t-t^{\prime}\right)}}+1+\frac{\sum_{q} e^{-\omega(q)\left(t+t^{\prime}\right)}}{\sum_{q} e^{-\omega(q)\left(t-t^{\prime}\right)}}\right\} .
\end{aligned}
$$

Através dessa relação podemos considerar três tipos de dinâmicas: supercrítica, crítica e subcrítica, classificadas com respeito à temperatura do sistema quando comparada a $T_{c}$. Na verdade não há sentido em estudar um regime dinâmico subcrítico do modelo gaussiano, já que esse não é definido abaixo da temperatura crítica. Restaria analisar os regimes crítico e supercrítico, porém, nesse último não se espera observar nenhum 
comportamento anômalo, pois ele se faz num estado de "altas temperaturas", em que o equilíbrio seria restaurado em um intervalo de tempo relativamente pequeno. Vamos então estudar um regime dinâmico crítico, que é observado nas imediatas vizinhanças de $q=0$.

Sabe-se que, quando um sistema se aproxima de seu ponto crítico seus tempos de relaxação vão ficando muito grandes. Dessa forma, uma hipótese plausível a respeito das funções de dois tempos é supor que elas decaiam por um tempo muito longo $t$, após $t^{\prime}$. Baseados nesse ponto analisaremos o caso $t>t^{\prime}$, com $t^{\prime} \rightarrow \infty$. De fato, outros regimes temporais poderiam ser analisados, por exemplo, regimes em que $t$ é da ordem de $t^{\prime}$, que por sua vez não vai ao infinito, isto é, um tempo de espera pequeno, quando comparado ao tempo que o sistema leva para atingir um estado de equilíbrio macroscópico. Investigações contemplando esse tipo de regime em geral não são muito interessantes. Estando o sistema muito distante do equilíbrio mesmo a formulação mais geral do TFD dada pela relação (3.32) pode não ser verificada. Provavelmente mesmo essa forma mais geral é somente válida para situações de um "quase equilíbrio termodinâmico".

Voltando à relação (3.33), consideremos o limite termodinâmico, usando então a transformação (2.22). Uma vez que investigaremos as regiões próximas a $q=0$, o estudo apenas na direção radial de $q$ é justificado, podendo ser feito através da equação (2.25). Para essa análise assintótica, $\omega(q)$ adquire a forma

$$
\omega(q) \sim J q^{2}+\frac{b}{\beta_{c}} \theta
$$

em que $\theta$ é a temperatura reduzida (isso para um modelo ferromagnético com interações de primeiros vizinhos). Note que estamos mudando a notação para não haver confusão com o tempo $t$. Podemos escrever o quociente de flutuação-dissipação como

$$
\begin{aligned}
X^{-1}=1 & +\left(1-b \theta\left\langle S^{2}(0)\right\rangle\right) e^{-\frac{b}{\beta_{c}} \theta t^{\prime}} \frac{\int_{\epsilon=0}^{a} d q q^{d-1} e^{-J\left(t+t^{\prime}\right) q^{2}}}{\int_{\epsilon=0}^{a} d q q^{d-1} e^{-J\left(t-t^{\prime}\right) q^{2}}} \\
& -J \beta_{c}\left\langle S^{2}(0)\right\rangle e^{-\frac{b}{\beta_{c}} \theta t^{\prime}} \frac{\int_{\epsilon=0}^{a} d q q^{d+1} e^{-J\left(t+t^{\prime}\right) q^{2}}}{\int_{\epsilon=0}^{a} d q q^{d-1} e^{-J\left(t-t^{\prime}\right) q^{2}}}
\end{aligned}
$$


Fazendo uso de uma transformação de variáveis,

$$
x=J\left(t+t^{\prime}\right) q^{2} \quad \text { e } \quad y=J\left(t-t^{\prime}\right) q^{2}
$$

temos,

$$
\begin{aligned}
X^{-1}=1+\left(1-\left\langle S^{2}(0)\right\rangle\right) & b \theta e^{-\frac{b}{\beta_{c}} \theta t^{\prime} \frac{\frac{\Gamma(d / 2)}{2\left[J\left(t+t^{\prime}\right)\right]^{\frac{d}{2}}}}{\frac{\Gamma(d / 2)}{2\left[J\left(t-t^{\prime}\right)\right]^{\frac{d}{2}}}}} \\
& -J \beta_{c}\left\langle S^{2}(0)\right\rangle e^{-\frac{b}{\beta_{c}} \theta t^{\prime}} \frac{\frac{\Gamma((d / 2)+1)}{2\left[J\left(t+t^{\prime}\right)\right]^{((d / 2)+1)}}}{\frac{\Gamma(d / 2)}{2\left[J\left(t-t^{\prime}\right)\right]^{d / 2}}} .
\end{aligned}
$$

Usando a relação $\Gamma(N+1)=N \Gamma(N)$, e levando em conta que estamos interessados em tempos longos, uma análise rápida mostra que o teorema de flutuação-dissipação é satisfeito, ou seja, no limite de $t^{\prime} \rightarrow \infty$, com $t>t^{\prime}$, temos

$$
X \rightarrow 1
$$

Isso indica que os mecanismos de flutuações térmicas e dissipação de energia, em um cenário dinâmico para o modelo gaussiano, num regime de tempos longos, relacionam-se da mesma forma que no estado de equilíbrio termodinâmico. No trababalho de (Hase, 2005) encontramos uma situação da não verificação do teorema de flutuação-dissipação nas mesmas condições aqui consideradas, porêm, para o modelo esférico.

\subsection{Modelo gaussiano crítico na dinâmica de Cahn-Hilliard}

A dinâmica de Cahn-Hilliard ou dinâmica do tipo B (Cahn e Hilliard, 1958; Hohenberg e Halperin, 1977) descreve a evolução temporal de um parâmetro de ordem conservado. Essa situação se refere a um sistema com um parâmetro de ordem, cuja integral espacial não varia no decorrer do tempo. Tal comportamento é traduzido, para um parâmetro de ordem $\phi$, por

$$
\frac{d}{d t} \int d^{d} r \phi(r, t)=0
$$

Essa classe de dinâmica foi desenvolvida num contexto de estudo de ligas binárias fundidas, que estão inicialmente a uma temperatura alta e são bruscamente resfriadas a uma temperatura inferior à sua temperatura de Curie. Um estado metaestável é então formado, e nele coexistem duas fases, com concentrações bem definidas. A evolução dinâmica desse 
sistema em que a diferença entre as concentrações das duas fases é conservada, é governada pela equação de Cahn-Hilliard, que é nada mais que uma equação de continuidade, que o parâmetro de ordem conservado deve seguir.

A presença de um vínculo desse tipo no sistema torna os cálculos para essa dinâmica mais complicados. Para inserirmos no modelo gaussiano esse comportamento dinâmico, devemos considerar uma dependência contínua das variáveis de spin em relação ao espaço. Usaremos então uma mudança de notação, $S_{\vec{r}}(t) \rightarrow S(\vec{r}, t)$.

A dinâmica de Cahn-Hilliard para o modelo gaussiano, que seria uma evolução dinâmica com uma magnetização média constante, é então representada pela equação

$$
\frac{\partial S(\vec{r}, t)}{\partial t}=\nabla^{2} \frac{\delta \mathcal{H}^{\prime}}{\delta S(\vec{r}, t)}+\eta(\vec{r}, t)
$$

com o hamiltoniano efetivo $\left(\mathcal{H}^{\prime}\right)$ dado por

$$
\mathcal{H}^{\prime}(\beta, t)=-\frac{1}{2} \int_{\mathcal{R}} d^{d} r \int_{\mathcal{R}} d^{d} r^{\prime} J\left(\left|r-r^{\prime}\right|\right) S(r, t) S\left(r^{\prime}, t\right)+\frac{b}{2 \beta} \int_{\mathcal{R}} d^{d} r[S(r, t)]^{2},
$$

e ainda com o ruído, que também é uma variável contínua, obedecendo

$$
\langle\eta(\vec{r}, t)\rangle=0 \quad e \quad\left\langle\eta(\vec{r}, t) \eta\left(\vec{r}^{\prime}, t^{\prime}\right)\right\rangle=-\frac{2}{\beta} \nabla^{2} \delta\left(\vec{r}-\vec{r}^{\prime}\right) \delta\left(t-t^{\prime}\right) .
$$

A extranha relação associada ao segundo momento da força aleatória é construida de tal forma a garantir que, para um tempo infinito, o teorema da aquipartição da energia seja recuperado, ou seja, que para tempos infinitos o sistema volte a obedecer os estados de Gibbs (equilíbrio). Essa relação pode ser facilmente verificada no espaço de Fourier.

Calculando a derivada funcional com respeito ao campo $S(r, t)$ temos

$$
\frac{\delta \mathcal{H}^{\prime}}{\delta S(r, t)}=-\int_{\mathcal{R}} d^{d} r^{\prime} J\left(\left|r-r^{\prime}\right|\right) S\left(r^{\prime}, t\right)+\frac{b}{\beta} S(r, t) .
$$

Uma vez que trabalhar no espaço dual é mais simples, pois o desacoplamento entre as variáveis se faz essencial para proseguirmos, iremos inserir o último resultado na equação (3.40) e tomar a transformada de Fourier (agora contínua), lembrando que $h=r-r^{\prime}$. Assim encontramos

$$
\frac{\partial \hat{S}_{q}(t)}{\partial t}=q^{2}\left[\hat{J}(q)-\frac{b}{\beta}\right] \hat{S}_{q}(t)+\hat{\eta}_{q}(t) .
$$

No espaço recíproco a equação (3.42) é dada por

$$
\left\langle\hat{\eta}_{q}(t) \hat{\eta}_{q^{\prime}}\left(t^{\prime}\right)\right\rangle=\frac{2 q^{2}}{\beta} \delta\left(q+q^{\prime}\right) \delta\left(t-t^{\prime}\right) .
$$


A solução da equação diferencial não-homogênea (3.44) é dada por

$$
\hat{S}_{q}(t)=\hat{S}_{q}(0) e^{-q^{2} \omega(q) t}+\int_{0}^{t} e^{-q^{2} \omega(q)\left(t-t_{1}\right)} \hat{\eta}_{q}\left(t_{1}\right) d t_{1}
$$

onde $\omega(q)$ é fornecido pela equação (3.15).

Vamos calcular a função de auto-correlação, que foi definida para spins discretos pela equação (3.12). No limite termodinâmico podemos reescrever a equação de autocorrelação de uma maneira mais adequada ao modelo $B$,

$$
C\left(t, t^{\prime}\right)=\frac{1}{(2 \pi)^{d}} \int d^{d} q\left\langle\hat{S}_{q}(t) \hat{S}_{-q}\left(t^{\prime}\right)\right\rangle
$$

Substituindo a equação (3.46) na última expressão, e fazendo uso da equação (3.45), da nulidade da média temporal do ruído e da condição inicial que foi considerada antes, temos

$$
\begin{aligned}
C\left(t, t^{\prime}\right) & =\frac{1}{(2 \pi)^{d}} \int_{\mathcal{R}} d^{d} q\left[\left\langle\hat{S}_{q}(0) \hat{S}_{-q}(0)\right\rangle e^{-q^{2} \omega(q)\left(t+t^{\prime}\right)}+\frac{2 q^{2}}{\beta} e^{-q^{2} \omega(q)\left(t+t^{\prime}\right)} \int_{0}^{t} d t_{1} e^{2 q^{2} \omega(q) t_{1}} \theta\left(t^{\prime}-t_{1}\right)\right] \\
& =\frac{1}{(2 \pi)^{d}} \int d^{d} q\left\{\left\langle S^{2}(0)\right\rangle e^{-q^{2} \omega(q)\left(t+t^{\prime}\right)}+\frac{1}{\beta \omega(q)}\left[e^{-q^{2} \omega(q)\left(t-t^{\prime}\right)}-e^{-q^{2} \omega(q)\left(t+t^{\prime}\right)}\right]\right\}(3.48)
\end{aligned}
$$

Evoluindo na mesma forma da secção anterior, vamos calcular a função resposta nesse sistema de infinitos campos. O hamiltoniano efetivo que agora devemos considerar toma a forma

$\mathcal{H}^{\prime}=-\frac{1}{2} \int_{\mathcal{R}} d^{d} r \int_{\mathcal{R}} d^{r} r^{\prime} J\left(\left|r-r^{\prime}\right|\right) S(r, t) S\left(r^{\prime}, t\right)-\int_{\mathcal{R}} d^{d} r H(r, t) S(r, t)+\frac{b}{2 \beta} \int_{\mathcal{R}}[S(r, t)]^{2} d^{d} r$,

onde $H(r, t)$ é um campo externo teste, com intensidade muito pequena. Inserindo a última relação na equação de Cahn-Hilliard e em seguida tomando a transformada de Fourier, obtemos

$$
\frac{\partial \hat{S}_{q}(t)}{\partial t}=-q^{2}\left\{\left[\frac{b}{\beta}-\hat{J}(q)\right] \hat{S}_{q}(t)-\hat{H}_{q}(t)\right\}+\hat{\eta}_{q}(t)
$$

onde

$$
\hat{H}_{q}(t)=\frac{1}{(2 \pi)^{d / 2}} \int_{\mathcal{R}} d^{d} r e^{-i q r} H(r, t) .
$$

A solução formal da última equação é dada por

$$
\hat{S}_{q}(t)=\hat{S}_{q}(0) e^{-q^{2} \omega(q) t}+e^{-q^{2} \omega(q) t} \int_{0}^{t} e^{q^{2} \omega(q) t_{1}}\left[q^{2} \hat{H}_{q}\left(t_{1}\right)+\hat{\eta}_{q}\left(t_{1}\right)\right] d t_{1} .
$$


O próximo passo consiste em calcular o valor médio de $\hat{S}_{q}(t)$ com a condição inicial já mencionada. Assim temos

$$
\left\langle\hat{S}_{q}(t)\right\rangle=e^{-q^{2} \omega(q) t} \int_{0}^{t} e^{q^{2} \omega(q) t_{1}} q^{2} \hat{H}_{q}\left(t_{1}\right) d t_{1} .
$$

Vamos escrever a função resposta para esse caso contínuo,

$$
R\left(t, t^{\prime}\right)=\frac{1}{(2 \pi)^{d}} \int d^{d} q\left[\frac{\delta\left\langle\hat{S}_{q}(t)\right\rangle}{\delta \hat{H}\left(t^{\prime}\right)}\right]_{\hat{H}=0} .
$$

Realizando uma derivada funcional do mesmo tipo da secção anterior encontramos

$$
R\left(t, t^{\prime}\right)=\frac{1}{(2 \pi)^{d}} \int d^{d} q q^{2} e^{-q^{2} \omega(q)\left(t-t^{\prime}\right)} .
$$

Os observáveis de interesse para a dinâmica de Cahn-Hilliard já foram encontrados. Devemos, então, analisar como eles se relacionam, se mantêm a interrelação do equilíbrio ou se isso é modificado no comportamento dinâmico. Passamos então a calcular a relação de flutuação-dissipação (3.32). Mais uma vez o regime de tempo que usaremos é $t>t^{\prime}$ $\operatorname{com} t^{\prime} \rightarrow \infty$.

Fazendo as substituições devidas, com $k_{B}=1$, podemos escrever

$$
X^{-1}=1-\frac{\left\langle S^{2}(0)\right\rangle}{T} \frac{\int d^{d} q q^{2} \omega(q) e^{-q^{2} \omega(q)\left(t+t^{\prime}\right)}}{\int d^{d} q^{2} e^{-q^{2} \omega(q)\left(t-t^{\prime}\right)}}+\frac{\int d^{d} q q^{2} e^{-q^{2} \omega(q)\left(t+t^{\prime}\right)}}{\int d^{d} q q^{2} e^{-q^{2} \omega(q)\left(t-t^{\prime}\right)}} .
$$

Outra vez o regime que nos interessa é o regime crítico. Então, em uma primeira instância, estudaremos um sistema que está na temperatura crítica. Assim, devemos substituir $\omega(q)$ pelo seu valor assintótico, $\omega\left(T_{c}, q=0\right) \rightarrow J q^{2}+\left(\mathcal{O} q^{4}\right)$. Sabendo que o comportamento depende apenas do módulo de $q$ podemos mais uma vez analisar somente a parte radial da equação (3.55). Então escrevemos

$$
X^{-1}=1-\frac{J\left\langle S^{2}(0)\right\rangle}{T_{c}} \frac{\int_{\epsilon=0}^{a} d q q^{d+3} e^{-J q^{4}\left(t+t^{\prime}\right)}}{\int_{\epsilon=0}^{a} d q q^{d+1} e^{-J q^{4}\left(t-t^{\prime}\right)}}+\frac{\int_{\epsilon=0}^{a} d q q^{d+1} e^{-J q^{4}\left(t+t^{\prime}\right)}}{\int_{\epsilon=0}^{a} d q q^{d+1} e^{-J q^{4}\left(t-t^{\prime}\right)}} .
$$

Fazendo uso de

$$
x=J q^{4}\left(t-t^{\prime}\right) \quad e \quad y=J q^{4}\left(t+t^{\prime}\right)
$$

temos

$$
X^{-1}=1-\frac{J^{\frac{1}{2}}\left\langle S^{2}(0)\right\rangle\left(t-t^{\prime}\right)^{\frac{d}{4}+\frac{1}{2}} \int_{0}^{J a^{4}\left(t+t^{\prime}\right)} d y y^{\frac{d}{4}} e^{-y}}{T_{c}\left(t+t^{\prime}\right)^{\frac{d}{4}+1} \int_{0}^{J a^{4}\left(t-t^{\prime}\right)} d x x^{\frac{d}{4}-\frac{1}{2}} e^{-x}}+\frac{\left(t-t^{\prime}\right)^{\frac{d}{4}+\frac{1}{2}} \int_{0}^{J a^{4}\left(t+t^{\prime}\right)} d y y^{\frac{d}{4}+\frac{1}{2}} e^{-y}}{\left(t+t^{\prime}\right)^{\frac{d}{4}+\frac{1}{2}} \int_{0}^{J a^{4}\left(t-t^{\prime}\right)} d x x^{\frac{d}{4}-\frac{1}{2}} e^{-x}} .
$$


Uma vez que estamos em um limite assintótico, a contribuição das integrais se dá quase que exclusivamente nas regiões imediatamente próximas à origem. Podemos então fazer os limites superiores das integrais irem ao infinito. Isso permite introduzir funções gama e escrever

$$
X^{-1}=1-\frac{J^{\frac{1}{2}}\left\langle S^{2}(0)\right\rangle\left(t-t^{\prime}\right)^{\frac{d}{4}+\frac{1}{2}} \Gamma\left(\frac{d}{4}+1\right)}{T_{c}\left(t+t^{\prime}\right)^{\frac{d}{4}+1} \Gamma\left(\frac{d}{4}+\frac{1}{2}\right)}+\frac{\left(t-t^{\prime}\right)^{\frac{d}{4}+\frac{1}{2}} \Gamma\left(\frac{d}{4}+\frac{1}{2}\right)}{\left(t+t^{\prime}\right)^{\frac{d}{4}+\frac{1}{2}} \Gamma\left(\frac{d}{4}+\frac{1}{2}\right)} .
$$

Considerando o regime de tempo $t>t^{\prime}$ com $t^{\prime} \rightarrow+\infty$, torna-se direto verificar que o teorema de flutuação-dissipação é satisfeito, ou seja, $X=1$.

Infelizmente, até o momento não conseguimos verificar se o TFD é válido nas proximidades da temperatura crítica $\left(T \rightarrow T_{c}^{+}\right)$do modelo gaussiano, que evolui no tempo segundo a dinâmica do tipo B.

\subsection{Formalismo MSR}

No tocante à dinâmica do modelo gaussiano, estudamos até agora formas padrão de obter as funções de autocorrelação e resposta. Fizemos cálculos, para as dinâmicas do tipo A e B, analisando a forma dessas funções na temperatura crítica e em suas proximidades.

Nesse ponto passamos a aplicar um método mais poderoso, desenvolvido por Martin, Siggia e Rose em um contexto muito mais amplo de teorias de campos (Martin et al., 1973) (Dominicis e Giardina, 2006) (Hertz, 1998). Quem primeiro aplicou tal procedimento ao contexto da mecânica estatística, com intuito, sobretudo, de tratar sistemas com desordem fixa, foi De Dominicis (Dominicis, 1976). Esse método se mostrará útil e prático para o estudo da dinâmica estocástica do modelo gaussiano segundo um enfoque diferente fornecido pela mesma equação de Langevin usada anteriormente.

Vamos aqui de início fazer uma explanação sucinta do método (Hertz, 1998), com um comentário final sobre a aplicação da álgebra de Grassmann, que fornecerá uma outra alternativa para obter a função resposta. Em seguida vamos aplicá-lo ao modelo gaussiano, comparando com os resultados obtidos anteriormente. 


\subsubsection{O método}

O nosso ponto de partida é a equação de Langevin, que pode ser escrita na forma

$$
\frac{d \phi}{d t}=-f(\phi)+\eta(t)
$$

onde $f(\phi)$ é uma força determinística e $\eta(t)$ é um ruído branco. Para cada ponto do sistema o ruído obedece as relações

$$
\langle\eta(t)\rangle=0 \quad \text { e } \quad\left\langle\eta(t) \eta\left(t^{\prime}\right)\right\rangle=2 T \delta\left(t-t^{\prime}\right)
$$

Dessa forma, a distribuição do ruído, devidamente normalizada (fato de suma importâcia), é expressa por

$$
W=\int D \eta(t) \exp \left[-\frac{1}{4 T} \int d t \eta^{2}(t)\right]=1
$$

Para compreendermos o significado da integração funcional realizada acima, vamos discretizar o tempo e escrever

$$
\int d t \eta^{2}(t) \rightarrow \lim _{\Delta t \rightarrow 0} \sum_{i} \eta^{2}\left(t_{i}\right) \Delta t
$$

Definindo

$$
D \eta(t)=\lim _{\Delta t \rightarrow 0} \prod_{i} \frac{d \eta\left(t_{i}\right) \sqrt{\Delta t}}{2 \sqrt{\pi T}}
$$

temos

$$
W=\int \lim _{\Delta t \rightarrow 0} \prod_{i} \frac{d \eta\left(t_{i}\right) \sqrt{\Delta t}}{2 \sqrt{\pi T}} \exp \left[-\frac{1}{4 T} \sum_{i}\left[\eta\left(t_{i}\right)\right]^{2} \Delta t\right] .
$$

Pode-se facilmente verificar a normalização dessa última expressão.

Usando a equação de Langevin é possível construir uma distribuição para o campo $\phi$ através da distribuição do ruído, deixando de trabalhar com uma variável estocástica em favor de uma variável determinística. Toma-se então a transformação

$$
\eta(t)=\dot{\phi}+f(\phi)
$$

levando em conta que

$$
D \eta(t)=J D \phi(t)
$$

onde $J$ é o jacobiano dessa transformação de variáveis. Portanto, uma distribuição para o campo $\phi$ pode ser expressa como

$$
P[\phi]=J \exp \left\{-\frac{1}{4 T} \int d t[\dot{\phi}+f(\phi)]^{2}\right\} .
$$


Vamos tentar dar uma idéia intuitiva de como encontrar o jacobiano J. Passamos então a considerar a relação

$$
\eta[\phi(t)]=\dot{\phi}+f[\phi(t)]
$$

sendo $\eta$ o ruído em um dado instante $t$. Podemos afirmar, sem complicações, que o ruído é constituído de duas funções independentes, ou seja,

$$
\eta=\eta\left\{\partial_{t} \phi(t) ; f[\phi(t)]\right\}
$$

Nesse sentido, devido à "memória" das forças estocásticas, o ruído em um dado tempo $t$ qualquer, pode ser expresso por

$$
\eta[\phi(t)]=\int_{0}^{t} d t^{\prime} \eta\left\{\partial_{t^{\prime}} \phi\left(t^{\prime}\right) ; f\left[\phi\left(t^{\prime}\right)\right]\right\}
$$

Calculando a variação do ruído com respeito ao campo em um tempo $t^{\prime \prime}$, temos

$$
\begin{aligned}
\frac{\delta \eta[\phi(t)]}{\delta \phi\left(t^{\prime \prime}\right)} & =\int d t^{\prime}\left\{\frac{\partial \eta}{\partial\left[\partial_{t^{\prime}} \phi\right]} \frac{\delta\left[\partial_{t^{\prime}} \phi\left(t^{\prime}\right)\right]}{\delta \phi\left(t^{\prime \prime}\right)}+\frac{\partial \eta}{\partial[f[\phi]]} \frac{\delta f\left[\phi\left(t^{\prime}\right)\right]}{\delta \phi\left(t^{\prime \prime}\right)}\right\} \\
& =\int d t^{\prime}\left\{\frac{\partial \eta}{\partial\left[\partial_{t^{\prime}} \phi\right]} \partial_{t^{\prime}} \delta\left(t^{\prime}-t^{\prime \prime}\right)+\frac{\partial \eta}{\partial[f[\phi]]} \frac{\partial f[\phi]}{\partial \phi} \delta\left(t^{\prime}-t^{\prime \prime}\right)\right\} \\
& =\frac{\partial \eta}{\partial\left[\partial_{t^{\prime \prime}} \phi\right]} \partial_{t^{\prime \prime}}+\frac{\partial \eta}{\partial[f[\phi]]} f^{\prime}\left[\phi\left(t^{\prime \prime}\right)\right] \\
& =\partial_{t^{\prime \prime}}+f^{\prime}\left[\phi\left(t^{\prime \prime}\right)\right] .
\end{aligned}
$$

A última relação é obtida invertendo a ordem de certas derivadas e ainda usando a equação (3.69). Os detalhes de cálculos desse tipo podem ser encontrado em textos de teoria de campos. Nesses mesmos textos podemos encontrar que o jacobiano de transformações envolvendo campos, como a que desejamos tratar, pode ser escrito como

$$
J=\operatorname{det}\left(\frac{\delta \eta[\phi]}{\delta \phi}\right)=\operatorname{det}\left\{\partial_{t}+f^{\prime}[\phi]\right\}
$$

Como exemplo, consideremos uma mola anarmônica, cuja força é dada por

$$
f[\phi]=\underbrace{\mu \phi}_{\text {termo harmônico }}+\underbrace{\frac{g}{3 !} \phi^{3}}_{\text {perturbação }} .
$$

O jacobiano para esse tipo de força é dado por

$$
J=\operatorname{det}\left[\partial_{t}+\mu+\frac{g}{2} \phi^{2}\right]
$$


Daqui por diante, como forma de ilustrar a aplicação do formalismo MSR, iremos utilizar a mola anarmônica.

Deve ser enfatizado que a distribuição associada a $\phi$ é também normalizada,

$$
W=\int D \phi P[\phi]=1 .
$$

Os valores médios de funções do campo $\phi$ são dados por

$$
\langle F(\phi)\rangle=\int D \phi(t) F[\phi] P[\phi] .
$$

Na mecânica estatística de equilíbrio a função de partição é a função geratriz dos momentos (médias) das grandezas de interesse. A fim de definirmos uma função geratriz no formalismo MSR, que desempenhe o mesmo papel da função de partição, adicionamos um campo auxiliar $h$ (que não deve ser confundido com um campo magnético externo) à distribuição de $\phi$,

$$
P[\phi]=J \exp \left\{-\frac{1}{4 T} \int d t[\dot{\phi}+f[\phi]]^{2}+\int d t h(t) \phi(t)\right\} .
$$

Definimos, então, a função geratriz desejada,

$$
W[h]=\int D \phi(t) J \exp \left\{-\frac{1}{4 T} \int d t[\dot{\phi}+f[\phi]]^{2}+\int d t h(t) \phi(t)\right\} .
$$

Assim é direto escrever

$$
\left\langle\phi\left(t_{1}\right) \phi\left(t_{2}\right) \cdots \phi\left(t_{n}\right)\right\rangle=\frac{\delta^{n} W[h]}{\delta h\left(t_{1}\right) \delta h\left(t_{2}\right) \cdots \delta h\left(t_{n}\right)} .
$$

A função geratriz no formalismo MSR, obtida anteriormente, é mais conveniente que uma função de partição. Os cálculos de médias no ensemble canônico envolvem derivadas do logaritmo da função de partição, enquanto agora, graças à normalização do ruído, as médias apenas envolvem derivadas da própria função geratriz. É nesse ponto que está a grande motivação e poder dos trabalhos desenvolvidos por De Dominicis sobre esse método. De Dominicis notou que tratando um sistema que possui desordem fixa pelo formalismo MSR, isso evita o uso do método de réplicas, e torna o procedimento de estudo mais simples e mais físico.

O passo seguinte no desenvolvimento do esquema MSR, operação essa fundamental, é eliminar o quadrado da expressão de $W[h]$ por meio da conhecida transformação gaussiana

$$
\exp \left(\frac{a^{2}}{2}\right)=\int_{-\infty}^{\infty} \frac{d x}{\sqrt{2 \pi}} \exp \left(-\frac{x^{2}}{2}+a x\right)
$$


dando origem a novas variáveis de campo $a(t)$. Então, temos

$$
W[h]=\int D \phi(t) J \exp \left\{\int h(t) \phi(t)\right\} \exp \left\{-\frac{1}{4 T} \int d t[\dot{\phi}+f(\phi)]^{2}\right\} .
$$

O argumento da exponencial que possui o termo quadrático, pode ser discretizado. Então

$$
\begin{aligned}
& \exp \left\{-\frac{1}{4 T} \int d t[\dot{\phi}+f(\phi)]^{2}\right\} \rightarrow \lim _{\Delta t \rightarrow 0} \prod_{i} \exp \left\{-\frac{\Delta t}{4 T}\left[\dot{\phi}\left(t_{i}\right)+f\left[\phi\left(t_{i}\right)\right]\right]^{2}\right\} \\
& =\lim _{\Delta \rightarrow 0} \prod_{i} \int \frac{d a\left(t_{i}\right)}{\sqrt{2 \pi}} \exp \left\{-\frac{a^{2}\left(t_{i}\right)}{2}+\left[\frac{i \sqrt{\Delta t}}{\sqrt{2 T}}\left[\dot{\phi}\left(t_{i}\right)+f\left(\phi\left(t_{i}\right)\right)\right]\right] a\left(t_{i}\right)\right\} .
\end{aligned}
$$

Com o intuito de deixar a expressão anterior um pouco mais "organizada", realizaremos uma outra transformação de variáveis, dada por

$$
a\left(t_{i}\right)=-\sqrt{2 T \Delta t} \psi\left(t_{i}\right)
$$

Colocando essa transformação na penúltima relação (deve ser lembrado que os limites de integração também se invertem com essa transformação), temos

$$
\begin{aligned}
& \lim _{\Delta \rightarrow 0}\left[\prod_{i} \int \frac{\sqrt{2 T \Delta t} d \psi\left(t_{i}\right)}{\sqrt{2 \pi}}\right] \exp \left\{-\sum_{i}\left[T \psi^{2}\left(t_{i}\right)+i\left[\dot{\phi}\left(t_{i}\right)+f\left[\phi\left(t_{i}\right)\right]\right] \psi\left(t_{i}\right)\right] \Delta t\right\} \\
= & \int D \psi(t) \exp \left\{-\int d t\left[T \psi^{2}(t)+i[\dot{\phi}(t)+f[\phi(t)]] \psi(t)\right]\right\} .
\end{aligned}
$$

Agora a função geratriz toma a forma

$$
W[h]=\int D \phi(t) D \psi(t) J \exp \left\{-\int d t\left[T \psi^{2}+i[\dot{\phi}+f(\phi)] \psi-h \phi\right]\right\} .
$$

Como já foi dito, vamos utilizar como exemplo, a mola anarmônica Assim temos

$$
W[h]=\int D \phi(t) D \psi(t) J \exp \left\{-\int d t\left[T \psi^{2}+i \dot{\phi} \psi+i \mu \phi \psi+\frac{i g}{3 !} \phi^{3} \psi-h \phi\right]\right\},
$$

onde $J$ é dado pela equação (3.75).

Não vamos fazer cálculos perturbativos de teoria de campos, pois esse não é o intuito desse trabalho. Dessa forma, da relação acima iremos reter apenas os termos de primeira ordem em $\phi$. Analisando até esse ponto estaremos aptos a estudar a dinâmica dissipativa do modelo gaussiano segundo o enfoque da técnica MSR. Os passos seguintes vão depender apenas do expoente da última relação, sem o termo de campo auxiliar h(t),

$$
A=-\int d t\left[T \psi^{2}(t)+i \dot{\phi} \psi+i \mu \phi \psi\right]
$$


Para tornar a relação acima mais simétrica, o que ficará mais claro em relações escritas um pouco mais à frente, adicionaremos à última expressão o termo $\int \frac{i}{2} d t \frac{d(\psi \phi)}{d t}$, cujo valor é nulo uma vez que o intervalo de integração é ] $-\infty,+\infty[$. Então

$$
A=-\frac{1}{2} \int d t\left[2 T \psi^{2}+i \dot{\phi} \psi+2 i \mu \phi \psi-i \phi \dot{\psi}\right]
$$

A expressão anterior pode ser escrita como uma forma matricial,

$$
A=-\frac{1}{2} \int d t\left(\begin{array}{ll}
\phi(t) & \psi(t)
\end{array}\right)\left(\begin{array}{cc}
0 & i \mu-i \partial_{t} \\
i \mu+i \partial_{t} & 2 T
\end{array}\right)\left(\begin{array}{l}
\phi(t) \\
\psi(t)
\end{array}\right) .
$$

É conveniente estudar essa relação no espaço de Fourier. Considerando

$$
\hat{\psi}(\omega)=\int_{-\infty}^{\infty} d t \psi(t) e^{i \omega t}
$$

Temos

$$
A=-\frac{1}{2} \int \frac{d \omega}{2 \pi}\left(\begin{array}{ll}
\hat{\phi}_{\omega} & \hat{\psi}_{\omega}
\end{array}\right) \underbrace{\left(\begin{array}{cc}
0 & i \mu+\omega \\
i \mu-\omega & 2 T
\end{array}\right)}_{M}\left(\begin{array}{c}
\hat{\phi}_{-\omega} \\
\hat{\psi}_{-\omega}
\end{array}\right) .
$$

Note que essa transformação é efetuada na penúltima relação; posteriormente nós a colocamos novamente na forma matricial.

Nesse ponto podemos escrever os segundos momentos entre as diversas variáveis, fazendo uso do teorema de Wick (uma pequena explanação sobre as ferramentas matemáticas aqui utilizadas e uma demonstração bastante intuitiva de uma forma básica do teorema de Wick se encontra no apêndice B). Esse teorema garante que os segundos momentos estão relacionados com a inversa da matriz $M$. Fazendo as correspondências corretas, e prestando atenção nos índices, temos

$$
\left(\begin{array}{cc}
\left\langle\hat{\phi}_{\omega} \hat{\phi}_{-\omega}\right\rangle & \left\langle\hat{\phi}_{\omega} \hat{\psi}_{-\omega}\right\rangle \\
\left\langle\hat{\psi}_{\omega} \hat{\phi}_{-\omega}\right\rangle & \left\langle\hat{\psi}_{\omega} \hat{\psi}_{-\omega}\right\rangle
\end{array}\right)=M^{-1}=\left(\begin{array}{cc}
\frac{2 T}{\omega^{2}+\mu^{2}} & \frac{1}{i \mu-\omega} \\
\frac{1}{i \mu+\omega} & 0
\end{array}\right) .
$$

O termo superior esquerdo da igualdade anterior corresponde à autocorrelação de um modelo livre no espaço recíproco. Tal resultado pode ser explicitado sem passar pelo formalismo MSR (Chaikin e Lubensky, 1995) (Haddad, 2004). O significado dos termos da diagonal secundária pode ser visto adicionando um campo magnético externo $-H^{\prime}$ à função $f[\phi]$. Esse campo faz surgir em nossa expressão para a ação sem termos 
perturbativos, ou seja, a relação para $A$, um ente adicional da forma $\int d t i \psi H^{\prime}$. Então, derivando uma média de $\phi$ com respeito a esse campo passamos a ter um segundo momento entre $\phi$ e $\psi$,

$$
\frac{\delta\langle\phi\rangle}{\delta H^{\prime}}=\langle i \psi \phi\rangle
$$

fica claro que, os termos presentes na diagonal secundária são funções do tipo resposta, a menos do fator $i$. Deve ser enfatizado novamente que esses resultados podem ser obtidos sem passar pelo esquema MSR (Chaikin e Lubensky, 1995) (Haddad, 2004).

Nesse ponto fica explícita uma das principais características dessa forma de estudar a dinâmica de sistemas sujeitos a forças de natureza estocástica. O método oferece as funções de autocorrelação e resposta ao mesmo tempo e da mesma forma.

Um último fato extremamente interessante é que os dois termos da diagonal secundária são funções do tipo resposta. O que isso significa? O termo superior dessa diagonal possui um pólo no semiplano superior complexo, indicando que se trata de uma função resposta causal, ou seja, que se anula para $t<t^{\prime}$; o termo inferior possui um pólo no semiplano inferior complexo, sendo então uma função resposta anticausal (Chaikin e Lubensky, 1995).

Com essa última verificação pode-se ainda perceber que a diferença entre as funções resposta causal e anticausal satisfaz a relação

$$
\hat{R}(\omega)-\hat{R}(-\omega)=-\frac{2 i \omega}{\omega^{2}+\mu^{2}}=\frac{-i \omega}{T} C(\omega) .
$$

Realizando uma antitransformada de Fourier, temos

$$
\dot{C}(t)=T R(t)
$$

que é a expressão do teorema de flutuação-dissipação, satisfeito para o caso de uma mola harmônica.

\subsubsection{Introdução dos campos de Grassmann}

Em física estamos acostumados a lidar com grandezas que comutam, associadas ao tipo de álgebra que aprendemos desde cedo. Porém, como seria uma álgebra onde as grandezas anti-comutam? Uma álgebra que lida exatamente com esse tipo de situação foi desenvolvida por Grassmann. 
A motivação de estudar uma álgebra de variáveis anti-comutantes se dá principalmente em teorias de campos fermiônicos. As funções de correlação são antisimétricas quanto à inversão da ordem do argumento. Então, quando desejamos escrever funcionais geradores gerais de funções de correlação, devemos usar variáveis anticomutantes, ou variáveis de Grassmann.

Em nosso contexto, a introdução dos campos de Grassmann nos permitirá lidar com o jacobiano da transformação obtida através da equação de Langevin. Para ilustrar algumas propriedades da álgebra grassmanniana, consideremos duas variáveis $\theta$ e $\bar{\theta}$, que por sua vez devem obedecer

$$
\theta \bar{\theta}=-\bar{\theta} \theta
$$

e

$$
\theta^{2}=\bar{\theta}^{2}=0
$$

Assim, das duas relações acima temos que toda expansão de uma função $f(\theta, \bar{\theta})$ é finita e da forma

$$
f(\theta, \bar{\theta})=a_{0}+a_{10} \theta+a_{01} \bar{\theta}+a_{11} \theta \bar{\theta}
$$

Ainda em álgebra grassmanniana as operações de derivação e integração não possuem nenhuma interpretação geométrica e não são defininas de forma a serem operações inversas, mas sim idênticas. Não reproduziremos aqui nada mais que algumas relações, que por sua vez representam como essas duas operações se dão nessa álgebra (para uma melhor análise da álgebra de Grassman vide (Carneiro e Thomaz, 2000) (Zinn-Justin, 1993)):

$$
\frac{\partial(1)}{\partial \theta}=\frac{\partial(1)}{\partial \bar{\theta}}=0, \quad \frac{\partial \bar{\theta}}{\partial \theta}=\frac{\partial \theta}{\partial \bar{\theta}}=0, \quad \text { e } \frac{\partial \theta}{\partial \theta}=\frac{\partial \bar{\theta}}{\partial \bar{\theta}}=1
$$

assim,

$$
\frac{\partial}{\partial \theta} \theta \bar{\theta}=\bar{\theta}, \quad \frac{\partial}{\partial \bar{\theta}} \bar{\theta} \theta=\theta, \quad \frac{\partial}{\partial \theta} \bar{\theta} \theta=-\bar{\theta} \quad \text { e } \frac{\partial}{\partial \bar{\theta}} \theta \bar{\theta}=-\theta .
$$

Uma vez que derivação e integração são definidas como sendo idênticas, temos

$$
\int d \theta d \bar{\theta}\left(\begin{array}{c}
1 \\
\theta \\
\bar{\theta} \\
\bar{\theta} \theta
\end{array}\right)=\left(\begin{array}{l}
0 \\
0 \\
0 \\
1
\end{array}\right) .
$$


Vale enfatizar a importância da ordem das variáveis a serem derivadas e integradas.

Passemos agora a direcionar o nosso estudo para as integrais gaussianas. Em particular podemos escrever

$$
e^{\bar{\theta} C \theta}=1+\bar{\theta} C \theta
$$

uma vez que os demais termos dessa expansão se anulam. Ainda consideremos a seguinte integral

$$
I(C)=\prod_{i}\left[\int d \theta_{i} d \bar{\theta}_{i}\right] \exp \left(\sum_{i, j=1}^{n} \bar{\theta}_{i} C_{i j} \theta_{j}\right) .
$$

Podemos então escrever

$$
\exp \left(\sum_{i, j=1}^{n} \bar{\theta}_{i} C_{i j} \theta_{j}\right)=\prod_{i, j=1}^{n} \exp \left(\bar{\theta}_{i} C_{i j} \theta_{j}\right)=\prod_{i, j=1}^{n}\left(1+\bar{\theta}_{i} C_{i j} \theta_{j}\right) .
$$

Colocando o último resultado na expressão de $I(C)$, podemos verificar, através das regras de integração e da regra de anticomutação, que os únicos termos que vão contribuir são os monômios que possuem todas as variáveis de Grassmann $\bar{\theta}_{i}$ e $\theta_{i}$. Uma maneira de lista-los consiste em escrever

$$
\sum_{\substack{\text { permutaçóes do conjunto } \\\left[j_{1}, j_{2} \cdots j_{n}\right]}} C_{1 j_{1}} C_{2 j_{2}} \cdots C_{n j_{n}} \bar{\theta}_{n} \theta_{j_{n}} \cdots \bar{\theta}_{1} \theta_{j_{1}} .
$$

Inserindo os resultados acima na equação (3.104), e realizando as permutações necessárias para a integração, percebemos em seguida que o resultado será dado pelo determinante de $C$. Uma ilustração pode ser feita, sem muito esforço, para o caso de duas variáveis de Grassmann.

Nesse ponto vamos tratar o jacobiano dado pela relação (3.73). Então é possível escrever

$$
J=\operatorname{det}\left[\partial_{t}+f^{\prime}[\phi]\right]=\int D \xi(t) D \bar{\xi}(t) \exp \left\{\int d t \bar{\xi}(t)\left[\partial_{t}+f^{\prime}[\phi]\right] \xi(t)\right\} .
$$

Assim, o funcional gerador $W$ passa a ter uma outra forma, que contém um par de campos reais e um outro par de campos de Grassmann,

$$
W=\int D \phi(t) D \psi(t) D \xi(t) D \bar{\xi}(t) e^{-\int d t \mathcal{L}(\phi, \psi, \xi, \bar{\xi})},
$$

com

$$
\mathcal{L}(\phi, \psi, \xi, \bar{\xi})=T \psi^{2}+i \psi[\dot{\phi}+f[\phi]]-\bar{\xi} \dot{\xi}-\bar{\xi} f^{\prime}[\phi] \xi
$$


Os campos de Grassmann são em geral denominados de campos "fantasmas".

Então, mais uma vez considerando o caso livre, ou seja, um sistema desprovido de termos perturbativos, podemos calcular funções de correlação entre os campos de Grassmann. Assim, no espaço recíproco dos tempos, temos

$$
\left\langle\xi_{-\omega} \bar{\xi}_{\omega}\right\rangle=\frac{\int d \xi_{-\omega} d \bar{\xi}_{\omega} \xi_{-\omega} \bar{\xi}_{\omega} e^{\bar{\xi}_{\omega}(i \omega+\mu) \xi_{\omega}}}{\int d \xi_{-\omega} d \bar{\xi}_{\omega} e^{\bar{\xi}_{\omega}(i \omega+\mu) \xi_{\omega}}}
$$

Devemos então expandir as exponenciais, usar a anticomutatividade das variáveis e em seguida as "regras" de integração. Finalmente temos

$$
\left\langle\xi_{-\omega} \bar{\xi}_{\omega}\right\rangle=-\frac{1}{i \omega+\mu}
$$

Fazendo uma comparação entre a última expressão e a equação (3.93), notamos que $\left\langle\xi_{-\omega} \bar{\xi}_{\omega}\right\rangle=-i\left\langle\hat{\phi}_{\omega} \hat{\psi}_{-\omega}\right\rangle$, é a função resposta causal. Assim fica apresentada uma outra forma de obter um observável de interesse.

\subsection{O modelo Gaussiano segundo o formalismo MSR}

Como já foi dito anteriormente, a dinâmica dissipativa do modelo gaussiano será estudada a partir de agora sob a luz do método desenvolvido por Martin, Siggia e Rose. Num primeiro momento realizaremos os cálculos que possibilitam encontrar as funções de correlação e de resposta ao mesmo tempo, pelo método apresentado nas páginas precedentes.

Consideremos o hamiltoniano do modelo gaussiano em uma versão contínua, pois o novo método foi desenvolvido para variáveis expressas como campos. Para nossos fins consideramos o hamiltoniano efetivo

$$
\mathcal{H}^{\prime}=-\frac{1}{2} \int_{\mathcal{R}} d^{d} r \int_{\mathcal{R}} d^{d} r^{\prime} J\left(\left|r-r^{\prime}\right|\right) S(r, t) S\left(r^{\prime}, t\right)+\frac{b}{2 \beta} \int_{\mathcal{R}} S^{2}(r, t) .
$$

A próxima etapa consiste em escrever a equação de Langevin para esse hamiltoniano,

$$
\frac{\partial S(r, t)}{\partial t}=-\left\{-\int_{\mathcal{R}} d^{d} r^{\prime} J\left(\left|r-r^{\prime}\right|\right) S\left(r^{\prime}, t\right)+\frac{b}{\beta} S(r, t)\right\}+\eta(r, t)
$$

onde o ruído $\eta$ obedece as relações

$$
\langle\eta(r, t)\rangle=0 \quad e \quad\left\langle\eta(r, t) \eta\left(r^{\prime}, t^{\prime}\right)\right\rangle=2 T \delta\left(r-r^{\prime}\right) \delta\left(t-t^{\prime}\right)
$$


Enfatizamos que o caráter vetorial das variáveis não está sendo explicitado para não carregar a notação.

Com as informações provenientes das duas últimas expressões, sabemos que o ruído deve obedecer uma distribuição de probabilidades normalizada, ou seja,

$$
W=\int D \eta(r, t) \exp \left\{-\frac{1}{4 T} \int d^{d} r d t \eta^{2}(r, t)\right\}=1
$$

Devemos agora isolar o ruído na equação (3.112) e substituir na distribuição da força estocástica. Uma vez que no espaço de Fourier temos um grande desacoplamento de variáveis, faremos uma transformada de Fourier nas variáveis de espaço antes de efetuar os cálculos. Considerando algumas das relações anteriores, escritas no espaço dual, temos

$$
\begin{gathered}
\frac{\partial \hat{S}_{q}(t)}{\partial t}=-\left\{\frac{b}{\beta}-\hat{J}(q)\right\} \hat{S}_{q}(t)+\hat{\eta}_{q}(t), \\
\left\langle\hat{\eta}_{q}(t)\right\rangle=0, \quad\left\langle\hat{\eta}_{q}(t) \hat{\eta}_{q^{\prime}}\left(t^{\prime}\right)\right\rangle=2 T \delta\left(q+q^{\prime}\right) \delta\left(t-t^{\prime}\right), \\
W=\int D \hat{\eta}_{q}(t) \exp \left\{-\frac{1}{4 T} \int d^{d} q d t \hat{\eta}_{q}^{2}(t)\right\} .
\end{gathered}
$$

O jacobiano da transformação, proveniente da equação de Langevin, é dado por

$$
J=\operatorname{det}\left[\partial_{t}+\frac{b}{\beta}-\hat{J}(q)\right] .
$$

Então

$$
D \hat{\eta}_{q}(t)=J D \hat{S}_{q}(t)
$$

Passamos agora a ter uma nova distribuição normalizada para as variáveis de spin,

$$
W=\int D \hat{S}_{q}(t) J \exp \left\{-\frac{1}{4 T} \int d t\left[\partial_{t}+\left(\frac{b}{\beta}-\hat{J}(q)\right)\right]^{2} \hat{S}_{q}^{2}(t)+\int d t \hat{h}(t) \hat{S}_{q}(t)\right\}=1 .
$$

Na expressão acima já aparece o termo de campo auxiliar, o qual possibilita escrever médias por meio de derivadas. Aqui realizamos as transformações necessárias para eliminar a parte quadrática e também inserir o termo que deixará a expressão acima mais simétrica. Assim, a ação, ou seja, o expoente da última expressão, toma a forma

$$
A=-\frac{1}{2} \int d^{d} q d t\left[2 T \hat{\psi}_{q}^{2}+i \hat{\psi}_{q} \partial_{t} \hat{S}_{q}+2 i \hat{\psi}_{q}\left(\frac{b}{\beta}-\hat{J}(q)\right) \hat{S}_{q}(t)-i \hat{S}_{q} \partial_{t} \hat{\psi}_{q}\right]
$$


Essa relação pode ser escrita como

$$
A=-\frac{1}{2} \int d^{d} q d t\left(\hat{S}_{q}(t) \quad \hat{\psi}_{q}(t)\right)\left(\begin{array}{cc}
0 & -i \partial_{t}+i\left(\frac{b}{\beta}-\hat{J}(q)\right) \\
i \partial_{t}+i\left(\frac{b}{\beta}-\hat{J}(q)\right) & 2 T
\end{array}\right)\left(\begin{array}{c}
\hat{S}_{q}(t) \\
\hat{\psi}_{q}(t)
\end{array}\right)
$$

Fazendo uma transformação de Fourier no tempo, temos

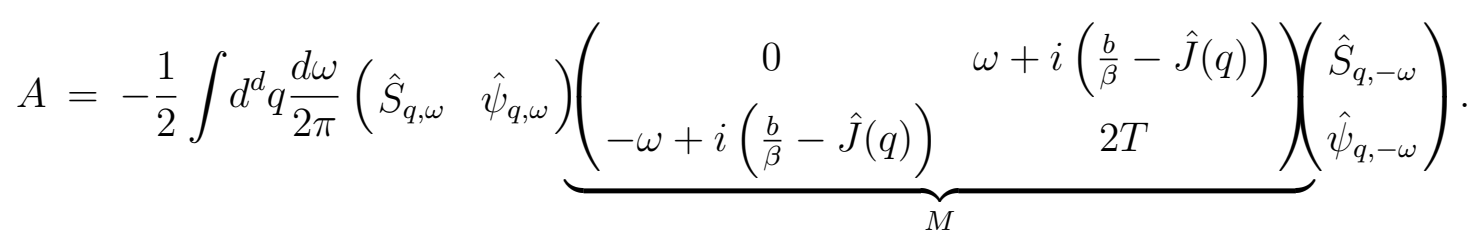

Como foi visto anteriormente, a inversa de $M$ está associada à função resposta e à função de correlação do modelo gaussiano. Então temos

$$
M^{-1}=\left(\begin{array}{cc}
\frac{2 T}{\omega^{2}+\left(\frac{b}{\beta}-\hat{J}(q)\right)^{2}} & \frac{1}{-\omega+i\left(\frac{b}{\beta}-\hat{J}(q)\right)} \\
\frac{1}{\omega+i\left(\frac{b}{\beta}-\hat{J}(q)\right)} & 0
\end{array}\right)=\left(\begin{array}{cc}
\left\langle\hat{S}_{q, \omega} \hat{S}_{q,-\omega}\right\rangle & \left\langle\hat{S}_{q, \omega} \hat{\psi}_{q,-\omega}\right\rangle \\
\left\langle\hat{\psi}_{q, \omega} \hat{S}_{q,-\omega}\right\rangle & \left\langle\hat{\psi}_{q, \omega} \hat{\psi}_{q,-\omega}\right\rangle
\end{array}\right)
$$

Assim como já foi dito, identificamos o termo superior da diagonal principal com a função de auto-correlação e o termo, também superior, da diagonal secundária com a função resposta (causal) do modelo gaussiano. O termo inferior da diagonal secundária é a função reposta anti-causal desse modelo.

Para não restar dúvida sobre a validade dos resultados encontrados acima vamos obtêlos sem passar pelo formalismo MSR. Para tanto consideremos

$$
\hat{S}_{q}(t)=\hat{S}_{q}(0) e^{-\omega^{\prime} t}+\int_{0}^{t} e^{-\omega^{\prime}\left(t-t^{\prime}\right)} \hat{\eta}_{q}\left(t^{\prime}\right) d t^{\prime}
$$

que é a solução já encontrada antes da equação de Langevin. Note que $\omega^{\prime}$ é o mesmo termo $(b / \beta)-\hat{J}(q)$, que mudou de notação, pois necessitamos realizar mais uma transformada de Fourier na variável tempo, que fará surgir a freqüência $\omega$. Após tal transformação de Fourier, temos

$$
\hat{S}_{q}(\omega)=\frac{\hat{S}_{q}(0)}{i \omega+\omega^{\prime}}+\frac{\hat{\eta}_{q}(\omega)}{i \omega+\omega^{\prime}} .
$$


Realizando esse mesmo tipo de transformação na correlação do ruído, temos

$$
\left\langle\hat{\eta}_{q}\left(\omega_{1}\right) \hat{\eta}_{q^{\prime}}\left(\omega_{2}\right)\right\rangle=2 T \delta\left(q+q^{\prime}\right) \delta\left(\omega_{1}+\omega_{2}\right) .
$$

A partir das duas últimas relações podemos calcular a auto-correlação. No espaço recíproco,

$$
\left\langle\hat{S}_{q}(\omega) \hat{S}_{-q}(-\omega)\right\rangle=\frac{\hat{S}(0)}{\omega^{2}+\omega^{\prime 2}}+\frac{2 T}{\omega^{2}+\omega^{\prime 2}} .
$$

Essa relação difere do termo superior da diagonal principal da expressão (3.124) somente pela primeira parcela, que por sua vez é associada à condição inicial. Tal fato é perfeitamente compreensível, pois o método MSR depende da distribuição do ruído, estando ligado ao comportamento após o início do processo dinâmico.

Para a função resposta, consideremos também a relação encontrada antes,

$$
\hat{R}_{q}\left(t, t^{\prime}\right)=e^{-\omega^{\prime}\left(t-t^{\prime}\right)}
$$

Também realizamos uma transformada de Fourier temporal, relembrando que a resposta a um estímulo externo deve ser causal $\left(t>t^{\prime}\right)$. Após a transformação, considerando a causalidade, temos

$$
\hat{R}_{q}(\omega)=\frac{1}{i \omega+\omega^{\prime}}
$$

que é exatamente $i\left\langle\hat{S}_{q, \omega} \hat{\psi}_{q,-\omega}\right\rangle$.

Fica assim mostrada a equivalência entre os dois métodos.

\subsection{O modelo gaussiano na presença de um campo aleatório}

Dizemos que um sistema de spins apresenta desordem quando as interações de troca são distribuídas aleatoriamente ou quando um campo externo age diferentemente em cada sítio da rede, de forma também aleatória. Essas variáveis (parâmetros de troca ou campos) são caracterizadas por uma dada distribuição de probabilidades. A desordem nas interações de troca é essencial para descrever o comportamento de materiais que apresentam uma fase tipo vidro de spin. No tocante à desordem existem dois tipos, "quenched", ou fixa, e "annealed", ou variável. Sob o ponto de vista experimental, uma desordem do tipo "quenched" significa que a escala dinâmica temporal da variação da 
desordem é muito maior que a escala de tempo das flutuações dos spins; uma desordem do tipo "annealed" significa que a escala temporal de variação da desordem é comparável à escala de flutuações das variáveis de spin.

Como já foi anunciado, o formalismo MSR é adequado ao estudo de sistemas que apresentam desordem fixa (do tipo "quenched"), substituindo o emprego do método de réplicas. Isso se deve à construção de uma função geratriz devidamente normalizada. Nesse sentido faremos um estudo muito simples do comportamento dinâmico do modelo gaussiano na presença de um campo aleatório. De início vamos mostrar o tipo de aproximação heurística que devemos fazer para "obter" o modelo gaussiano a partir do modelo de Ising. Isso se dá através da transformação de Hubbard,construindo um modelo de spins "macios" a partir do modelo de Ising. Tal modelo de spins "macios" é o ponto de partida de muitos trabalhos, apelando a argumentos de teorias de escala e universalidade, em sintonia com o contexto mais geral do hamiltoniano de Landau-Ginzburg. Vamos aqui mostrar a sua origem. Construído tal hamiltoniano, descartamos o termo quártico, recaindo no caso gaussiano que desejamos investigar.

O modelo de Ising na presença de um campo aleatório é definido através do hamiltoniano

$$
\mathcal{H}=-\frac{1}{2} \sum_{r, r^{\prime}} J_{r, r^{\prime}} S_{r} S_{r^{\prime}}-\sum_{r} H_{r} S_{r},
$$

com $S_{r}= \pm 1, J_{r, r^{\prime}}$ sendo a interação de troca entre dois sítios de uma rede hipercúbica d-dimensional, que por sua vez está sujeita a um campo magnético $H_{r}$, diferente para cada sítio.

A distribuição do campo aleatório se dá segundo a relação

$$
p\left(H_{r}\right)=\left(2 \pi H^{2}\right)^{-\frac{1}{2}} \exp \left[-\frac{H_{r}^{2}}{2 H^{2}}\right],
$$

onde $H^{2}$ é a variância. Um fato de suma importância é que essa desordem é do tipo "quenched", isto é, $H_{r}$ é constante na escala de tempo em que os graus de liberdade $S_{r}$ sofrem flutuações.

Para esse modelo de Ising, podemos pensar que temos uma energia livre para cada configuração de campo. Com efeito, toda grandeza termodinâmica encontrada deverá ser pesada, segundo a distribuição do campo [3.132]. Assim podemos escrever a função de 
partição para uma dada configuração do campo,

$$
Z_{\left\{H_{r}\right\}}=\operatorname{Tr} \exp \left\{\frac{1}{2} \sum_{r, r^{\prime}} K_{r, r^{\prime}} S_{r} S_{r^{\prime}}+\sum_{r} L_{r} S_{r}\right\},
$$

sendo que $\operatorname{Tr}$ representa a soma sobre todas as configurações possíveis das variáveis de spin, para uma dada configuração do campo aleatório; $K_{r, r^{\prime}}=\beta J_{r, r^{\prime}}$ e $L_{r}=\beta H_{r}$.

A última expressão pode ser escrita em uma forma matricial, o que tornará mais intuitivo a passagem seguinte,

$$
Z_{\left\{H_{r}\right\}}=\operatorname{Tr} \exp \left\{\frac{1}{2} \tilde{\mathbf{S}} \mathbf{K S}+\tilde{\mathbf{L}} \mathbf{S}\right\} .
$$

Tendo em vista que $\mathbf{K}$ é uma matriz real e simétrica, existe uma transformação unitária $\mathbf{U} \tilde{\mathbf{U}}=\mathbf{I}$, onde $\mathbf{I}$ é a matriz identidade, que diagonaliza $\mathbf{K}$. Então fazemos

$$
\begin{aligned}
Z_{\left\{H_{r}\right\}} & =\operatorname{Tr} \exp \left\{\frac{1}{2} \tilde{\mathbf{S}} \tilde{\mathbf{U K}} \tilde{\mathbf{U}} \mathbf{U S}+\tilde{\mathbf{L}} \tilde{\mathbf{U}} \mathbf{S}\right\} \\
& =\operatorname{Tr} \exp \left\{\sum_{r}\left[\frac{1}{2} \lambda_{r} \sigma_{r}^{2}+L_{r}^{\prime} \sigma_{r}\right]\right\},
\end{aligned}
$$

onde $\mathbf{U S}=\sigma, \mathbf{U L}=\mathbf{L}^{\prime}$, e ainda usamos o fato de que a transposta do produto de duas matrizes é o produto das transpostas com a ordem dessa multiplicação invertida.

Nesse ponto estamos aptos a realizar uma transformação gaussiana para eliminar os termos quadráticos em $\sigma_{r}$,

$$
Z_{\left\{H_{r}\right\}}=\operatorname{Tr} \prod_{r}\left\{\int_{-\infty}^{\infty} \frac{d x_{r}}{\sqrt{\pi}} \exp \left[-x_{r}^{2}+\sqrt{2 \lambda_{r}} \sigma_{r} x_{r}+L_{r}^{\prime} \sigma_{r}\right]\right\}
$$

Fazendo

$$
y_{r}=\sqrt{2 \lambda_{r}} x_{r}
$$

e escrevendo novamente em uma forma matricial, temos

$$
\begin{aligned}
Z_{\left\{H_{r}\right\}} & =\operatorname{Tr} \int_{-\infty}^{\infty} \exp \left\{-\frac{1}{2} \tilde{\mathbf{y}} \mathbf{D}^{-1} \mathbf{y}+\tilde{\mathbf{y}} \sigma+\tilde{\mathbf{L}^{\prime}} \sigma\right\} \prod_{r}\left[\frac{d y_{r}}{\sqrt{2 \pi \lambda_{r}}}\right] \\
& =\operatorname{Tr} \int_{-\infty}^{\infty} \exp \left\{-\frac{1}{2} \tilde{\mathbf{z}} \mathbf{K}^{-1} \mathbf{z}+(\tilde{\mathbf{z}}+\tilde{\mathbf{L}}) \mathbf{S}\right\} \prod_{r}\left[\frac{d z_{r}}{\sqrt{2 \pi \lambda_{r}}}\right] .
\end{aligned}
$$

Para obter a relação acima usamos o fato de que $\mathbf{D}^{-1}=\mathbf{U} \mathbf{K}^{-1} \tilde{\mathbf{U}}$, surgindo assim $\mathbf{z}=\tilde{\mathbf{U y}}$, que se trata de uma transformação unitária, com o jacobiano igual à unidade. Fazendo 
uma outra transformação dada por $\theta \equiv \mathbf{z}+\mathbf{L}$ e calculando o traço, temos

$$
\begin{aligned}
Z_{\left\{H_{r}\right\}} & =\operatorname{Tr} \int \exp \left\{-\frac{1}{2}(\tilde{\theta}-\tilde{\mathbf{L}}) \mathbf{K}^{-1}(\theta-\mathbf{L})+\tilde{\theta} \mathbf{S}\right\} \prod_{r}\left[\frac{d \theta_{r}}{\sqrt{2 \pi \lambda_{r}}}\right] \\
& =\frac{2^{N^{d} / 2}}{\pi^{N^{d} / 2 \sqrt{\operatorname{det} \mathbf{K}}}} \int \exp \left\{-\frac{1}{2}(\tilde{\theta}-\tilde{\mathbf{L}}) \mathbf{K}^{-1}(\theta-\mathbf{L})+\sum_{r} \ln \left[\cosh \theta_{r}\right]\right\} \prod_{r} d \theta_{r} \cdot(3 .
\end{aligned}
$$

Como última transformação, faremos $\theta=-\mathbf{K} \phi$ e ainda usaremos o fato de que a inversa de uma matriz simétrica também é simétrica. Assim, na forma de uma integral funcional, temos

$$
Z_{\left\{H_{r}\right\}}=\frac{2^{N^{d} / 2} e^{-\frac{1}{2} \tilde{\mathbf{L}} \mathbf{K}^{-1} \mathbf{L}}}{\pi^{N^{d} / 2} \sqrt{\operatorname{det} \mathbf{K}}} \int D \phi \exp \left\{-\frac{1}{2} \tilde{\phi} \mathbf{K} \phi-\tilde{\mathbf{L}} \phi+\sum_{r} \ln \left[\cosh \left(-\sum_{r^{\prime}} K_{r, r^{\prime}} \phi_{r^{\prime}}\right)\right]\right\} .
$$

Dessa forma, como a função cosh é par, encontramos que o hamiltoniano efetivo para esse modelo de Ising na presença de um campo aleátório é dado por

$$
\beta \mathcal{H}_{e f}=-\frac{1}{2} \sum_{r, r^{\prime}} K_{r, r^{\prime}} \phi_{r} \phi_{r^{\prime}}-\sum_{r} L_{r} \phi_{r}+\sum_{r} \ln \left[\cosh \theta_{r}\right]
$$

Qualquer estudo da dinâmica do modelo de Ising na presença de um campo aleatório deveria ser feito através do hamiltoniano efetivo acima. Porém, isso não é realizado, uma vez que o novo parâmetro de ordem, obtido pela transformação de Hubbard e pelas outras várias transformações, depende também da distribuição do campo $H_{r}$, dificultando muito os cálculos. Então, para evoluirmos necessitamos realizar uma série de aproximações e simplificações.

As nossas aproximações se guiarão de acordo com o contexto clássico de estudo das características termodinâmicas de um modelo nas vizinhanças da criticalidade, onde, segundo o esquema proposto por Landau, o módulo do parâmetro de ordem dever ser tão menor quanto mais próximo o sistema estiver de sua temperatura crítica. Então devemos fazer uma expansão do termo $\ln \cosh \theta_{r}$ em torno de $\theta_{r}=0$. Porém tal expansão poderia ter problemas, pois $\theta_{r}$ depende da variável aleatória $H_{r}$, que pode variar de ] $-\infty, \infty[$, não podendo assim $\theta_{r}$ ser tomado sempre pequeno. No entanto, a média da distribuição simétrica do campo $H_{r}$ é centrada em zero, o que justifica essa expansão. Portanto, estamos apresentando um tratamento que se justifica pela simetria do sistema. Realizando tal operação obtemos

$$
\ln \cosh \theta_{r}=\frac{1}{2} \theta_{r}^{2}-\frac{1}{12} \theta_{r}^{4}+\cdots
$$


Vamos reter apenas os dois primeiros termos da série.

Nessas considerações ainda se deve levar em conta a forma da interação de troca. Sabe-se que a interação de troca decai muito rapidamente com a distância. Então, sendo $J_{0}>0$ (caso ferromagnético) a interação de troca entre dois primeiros vizinhos da rede ddimensional que estamos considerando, as demais interações serão muito pequenas. Dessa forma através da transformação $\theta=-K \phi$, escrevemos

$$
\theta_{r} \cong-\left(2 d J_{o}+a\right) \phi_{r}=-r_{0}^{\frac{1}{2}} \phi_{r}
$$

onde d é a dimensionalidade da rede e $a$ é um fator, pequeno, que busca levar em conta os termos de interações entre os demais vizinhos do sítio $r$.

Por fim, fazendo $\phi_{r}=S_{r}$, temos

$$
\beta \mathcal{H}_{e f}=-\frac{1}{2} \sum_{r, r^{\prime}} K_{r, r^{\prime}} S_{r} S_{r^{\prime}}-\sum_{r} L_{r} S_{r}+\frac{r_{0}}{2} \sum_{r} S_{r}^{2}+u \sum_{r} S_{r}^{4} .
$$

Esse é um modelo de spins "macios". Esse hamiltoniano acima ainda poderia ser chamado de hamiltoniano de Landau-Ginzburg, onde o termo de interação entre dois sítios da rede corresponde ao termo que apresenta o laplaciano no hamiltoniano de L-G.

Um outro argumento usado para o emprego do hamiltoniano de spins macios, como informação inicial é que ele se trata do modelo gaussiano acrescido de um termo quártico, o que permite ir além da descrição usual de campo médio.

\subsubsection{Aplicação da técnica MSR}

Daqui por diante vamos utilizar o hamiltoniano do modelo de spins macios para estudar a aplicação da técnica MSR a uma situação com desordem do tipo "quenched". Num primeiro momento, vamos realizar os passos iniciais requeridos no formalismo MSR, para o modelo de spins macios na presença do campo aleatório, indicando os passos seguintes que devem ser feitos para tratar o modelo com o termo quártico. No caso gaussiano vamos calcular as funções resposta e de auto-correlação.

Nosso ponto de partida consiste em escrever a equação de Langevin usando o hamil- 
toniano efetivo dado pela relação [3.144],

$$
\begin{aligned}
\frac{\partial S_{r}(t)}{\partial t} & =-\frac{\delta \mathcal{H}_{e f}}{\delta S_{r}(t)}+\eta_{r}(t) \\
& =-T\left(-\sum_{r^{\prime}} K_{r, r^{\prime}} S_{r^{\prime}}-L_{r}+r_{0} S_{r}+4 u S_{r}^{3}\right)+\eta_{r}(t)
\end{aligned}
$$

com

$$
\left\langle\eta_{r}(t)\right\rangle=0, \quad\left\langle\eta_{r}(t) \eta_{r^{\prime}}\left(t^{\prime}\right)\right\rangle=2 T \delta_{r, r^{\prime}} \delta\left(t-t^{\prime}\right)
$$

Tendo em vista a forma da distribuição do campo dada pela expressão [3.132], podemos escrever

$$
\left\langle H_{r}\right\rangle=0, \quad\left\langle H_{r} H_{r^{\prime}}\right\rangle=H^{2} \delta_{r, r^{\prime}}
$$

Seguindo o processo habitual, uma vez que a distribuição do ruído é devidamente normalizada, e fazendo uso da equação de Langevin, podemos escrever a função geratriz

$$
\begin{aligned}
W_{H_{r}} & =\int D S_{r}(t) \operatorname{Jexp}\left\{-\frac{1}{4 T} \int d t \sum_{r}\left[\frac{\partial S_{r}(t)}{\partial t}-T\left(\sum_{r^{\prime}} K_{r, r^{\prime}} S_{r^{\prime}}(t)+L_{r}-r_{0} S_{r}(t)-4 u S_{r}(t)\right)\right]^{2}\right\} \\
& =1 .
\end{aligned}
$$

A relação anterior é rica, trazendo diversas informações importantes. Primeiramente, notamos que a função geratriz depende de uma dada configuração do campo aleatório, da mesma maneira que a função de partição. Logo ela deve ser pesada pela distribuição gaussiana do campo para cada sítio. Nota-se ainda que o jacobiano da transformação associada à equação de Langevin, dado por

$$
\begin{aligned}
J & =\operatorname{det}\left(\partial_{t}+\frac{\delta^{2} \mathcal{H}_{e f}}{\delta S_{r}^{2}(t)}\right) \\
& =\operatorname{det}\left[\partial_{t}+T\left(r_{0}+12 u S_{r}^{2}(t)\right)\right]
\end{aligned}
$$

não depende da desordem, ou seja, não depende de $H_{r}$. Esse ponto é de suma importância, pois permite um avanço mais fácil nos cálculos. Em adição a isso, essa independência do jacobiano com o campo garante que somente a função de auto-correlação deverá ser influenciada pela presença da desordem. Isso pode ser evidenciado através das variáveis de Grassmann, cuja formulação nos mostrou que a função resposta é obtida somente através do jacobiano.

O passo seguinte consiste em inserir a transformação gaussiana usual para eliminar o quadrado no expoente; feito isso, toma-se a média com respeito ao campo $H_{r}$, para aí 
sim calcular as funções de interesse. Porém, a presença do termo $S_{r}^{3}(t)$ no expoente de $W$ dificulta os cálculos e nos obrigaria a apelar às teorias de perturbação, que pretendemos por enquanto evitar.

Então, a partir de agora desprezaremos o termo quártico do hamiltoniano efetivo [3.144], recaindo no modelo gaussiano. Reescreveremos ainda esse hamiltoniano com uma dependência contínua das variáveis espaciais. Feitas as devidas correspondências, o sistema que desejamos investigar é descrito pelo hamiltoniano efetivo

$$
\mathcal{H}_{e f}=-\frac{1}{2} \int_{\mathcal{R}} d^{d} r d^{d} r^{\prime} J\left(\left|r-r^{\prime}\right|\right) S(r, t) S\left(r^{\prime}, t\right)+\frac{b}{2 \beta} \int_{\mathcal{R}} d^{d} S^{2}(r, t)-\int_{\mathcal{R}} d^{d} r H(r) S(r, t),
$$

onde as variáveis de spin podem variar em toda a reta real e $H(r)$ é uma variável aleatória, introduzindo no sistema uma desordem do tipo "quenched". A distribuição dos campos é gaussiana,

$$
\langle H(r)\rangle=0 \quad e \quad\left\langle H(r) H\left(r^{\prime}\right)\right\rangle=H^{2} \delta\left(r-r^{\prime}\right)
$$

A equação de Langevin do nosso sistema é dada por

$$
\frac{\partial S(r, t)}{\partial t}=-\left\{-\int_{\mathcal{R}} d^{d} r^{\prime} J\left(\left|r-r^{\prime}\right|\right) S\left(r^{\prime}, t\right)+\frac{b}{\beta} S(r, t)-H(r)\right\}+\eta(r, t),
$$

com

$$
\langle\eta(r, t)\rangle=0 \quad e \quad\left\langle\eta(r, t) \eta\left(r^{\prime}, t^{\prime}\right)\right\rangle=2 T \delta\left(r-r^{\prime}\right) \delta\left(t-t^{\prime}\right)
$$

Como já vimos, tratar o termo de interação de spins no espaço direto dificulta muito os cálculos. Devemos então escrever a última relação no espaço de Fourier, onde o acoplamento espacial é desfeito. Assim temos

$$
\frac{\partial \hat{S}_{q}(t)}{\partial t}=-\left\{\left(\frac{b}{\beta}-\hat{J}(q)\right) \hat{S}_{q}(t)-\hat{H}_{q}\right\}+\hat{\eta}_{q}(t)
$$

Ainda no espaço recíproco também temos

$$
\begin{array}{ll}
\left\langle\hat{\eta}_{q}(t)\right\rangle=0, & \left\langle\hat{\eta}_{q}(t) \hat{\eta}_{q^{\prime}}\left(t^{\prime}\right)\right\rangle=2 T \delta\left(q+q^{\prime}\right) \delta\left(t-t^{\prime}\right), \\
\left\langle\hat{H}_{q}\right\rangle=0 \quad e & \left\langle\hat{H}_{q} \hat{H}_{q^{\prime}}\right\rangle=H^{2} \delta\left(q+q^{\prime}\right),
\end{array}
$$

onde a transformada de Fourier do campo aleatório é defina por

$$
\hat{H}_{q}=\int \frac{d r}{(2 \pi)^{d / 2}} e^{-i q r} H(r)
$$


Sendo a distribuição do ruído normalizada, com o auxílio da equação de Langevin no espaço de Fourier escrevemos

$$
W_{\left\{H_{r}\right\}}=\int D \hat{S}_{q}(t) J \exp \left\{-\frac{1}{4 T} \int d t d^{d} q\left[\left(\partial_{t}+\frac{b}{\beta}-\hat{J}(q)\right) \hat{S}_{q}(t)-\hat{H}_{q}\right]^{2}\right\}
$$

onde o jacobiano, como já dissemos, é independente da desordem e a função geratriz é dependente da configuração da variável aleatória no instante estudado.

Seguindo o caminho natural empregado no uso do formalismo MSR, devemos eliminar o quadrado do expoente da exponencial da última relação através de uma transformação gaussiana. Essa transformação é realizada discretizando o tempo e o espaço e fará surgir um campo auxiliar $\hat{\sigma}_{q}(t)$ que é o preço pago pela linearização no parâmetro de ordem. Assim temos

$$
W_{\left\{\hat{H}_{q}\right\}}=\int D \hat{S}_{q}(t) D \hat{\sigma}_{q}(t) J \exp \left\{\int d^{d} q d t\left[-T \hat{\sigma}_{q}^{2}(t)-i \hat{\sigma}_{q}(t)\left(\partial_{t}+\frac{b}{\beta}-\hat{J}(q)\right) \hat{S}_{q}(t)+i \hat{\sigma}_{q}(t) \hat{H}_{q}\right]\right\} .
$$

No passo seguinte realizamos a média da função geratriz com respeito à distribuição do campo. Uma vez que a desordem é do tipo "quenched", devemos calcular

$$
\int D \hat{H}_{q} \exp \left\{-\frac{1}{2 H^{2}} \int d^{d} q \hat{H}_{q}^{2}+i \int d^{d} q d t \hat{H}_{q} \hat{\sigma}_{q}(t)\right\}
$$

que também pode ser obtida através de uma discretização do espaço (mesmo tipo de operação já apresentada previamente). De fato, devemos encontrar o valor de

$$
\int_{-\infty}^{\infty} \frac{d \hat{H}_{q}}{\sqrt{2 \pi H^{2}}} \exp \left\{-\frac{\hat{H}_{q}^{2} \Delta q}{2 H^{2}}+i \hat{H}_{q} \Delta q \int d t \hat{\sigma}_{q}(t)\right\} .
$$

Completando quadrados, efetuando a operação acima e tomando a média, temos a função geratriz

$$
\begin{gathered}
W=\int D \hat{S}_{q}(t) D \hat{\sigma}_{q}(t) J \exp \left\{\int d^{d} q d t\right. \\
{\left[-T \hat{\sigma}_{q}^{2}(t)-i \hat{\sigma}_{q}(t)\left(\partial_{t}+\frac{b}{\beta}-\hat{J}(q)\right) \hat{S}_{q}(t)\right]} \\
\left.-\frac{H^{2}}{2} \int d^{d} q\left[\int d t \hat{\sigma}_{q}(t)\right]^{2}\right\} .
\end{gathered}
$$

Pela expressão acima fica evidente que a desordem insere um acoplamento entre diferentes tempos. Nesse ponto percebe-se uma semelhança entre o comportamento dinâmico desse sistema desordenado e o seu tratamento estático, que é feito pelo método de réplicas. Isso significa que a desordem insere um acoplamento entre diferentes réplicas, ou seja, 
sítios que estavam inicialmente descorrelacionados. De Dominicis mostrou que o limite estático do caso dinâmico é exatamente o mesmo do caso termodinâmico, (Dominicis e Giardina, 2006).

A ação associada ao comportamento dinâmico do modelo gaussiano na presença de um campo aleatório pode ser expressa na forma

$$
\begin{aligned}
A & =-\frac{1}{2} \int d^{d} q d t d t^{\prime}\left\{2 T \hat{\sigma}_{q}(t) \hat{\sigma}_{q}\left(t^{\prime}\right) \delta\left(t-t^{\prime}\right)+i \hat{\sigma}_{q}(t) \partial_{t^{\prime}} \hat{S}\left(t^{\prime}\right) \delta\left(t-t^{\prime}\right)-i \hat{S}_{q}(t) \partial_{t^{\prime}} \sigma_{q}\left(t^{\prime}\right) \delta\left(t-t^{\prime}\right)\right. \\
& \left.+i \hat{\sigma}_{q}(t)\left(\frac{b}{\beta}-\hat{J}(q)\right) \hat{S}_{q}\left(t^{\prime}\right) \delta\left(t-t^{\prime}\right)+i \hat{S}_{q}(t)\left(\frac{b}{\beta}-\hat{J}(q)\right) \hat{\sigma}_{q}\left(t^{\prime}\right) \delta\left(t-t^{\prime}\right)+H^{2} \hat{\sigma}_{q}(t) \hat{\sigma}_{q}\left(t^{\prime}\right)\right\}
\end{aligned}
$$

onde um termo simetrizador, da mesma forma que já havia sido utilizado em secções anteriores, foi novamente empregado. Em virtude dessa simetrização podemos reescrever o argumento da integral da última expressão em uma forma matricial,

$$
\begin{aligned}
A & =-\frac{1}{2} \int d^{d} q d t d t^{\prime}\left(\begin{array}{cc}
\hat{S}_{q}(t) & \left.\hat{\sigma}_{q}(t)\right) \times \\
0 & i\left(-\partial_{t^{\prime}}+\left(\frac{b}{\beta}-\hat{J}(q)\right)\right) \delta\left(t-t^{\prime}\right) \\
& \times\left[\begin{array}{cc}
i\left(\partial_{t^{\prime}}+\left(\frac{b}{\beta}-\hat{J}(q)\right)\right) \delta\left(t-t^{\prime}\right) & 2 T \delta\left(t-t^{\prime}\right)+H^{2}
\end{array}\right]\left(\begin{array}{l}
\hat{S}_{q}\left(t^{\prime}\right) \\
\hat{\sigma}_{q}\left(t^{\prime}\right)
\end{array}\right) .
\end{array}\right.
\end{aligned}
$$

Realizando uma transformada de Fourier temporal,

$$
\hat{S}_{q}(t)=\int \frac{d \omega}{2 \pi} \hat{S}_{q, \omega} e^{-i \omega t}, \quad \hat{\sigma}_{q}(t)=\int \frac{d \omega}{2 \pi} \hat{\sigma}_{q, \omega} e^{i \omega t}
$$

temos

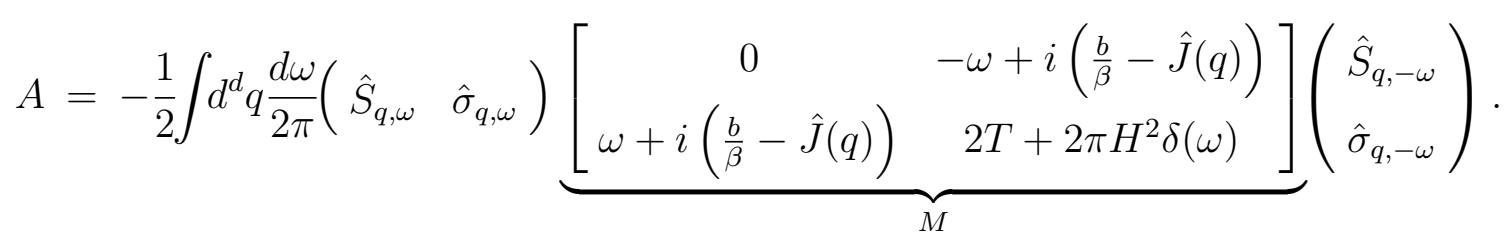

Como já foi visto, as funções de auto-correlação e resposta estão associadas à inversa da matriz $M$. Por fim encontramos

$$
M^{-1}=\left[\begin{array}{cc}
\frac{2 T+2 \pi H^{2} \delta(\omega)}{\omega^{2}+\left(\frac{b}{\beta}-\hat{J}(q)\right)^{2}} & \left(\omega+i\left(\frac{b}{\beta}-\hat{J}(q)\right)\right)^{-1} \\
\left(-\omega+i\left(\frac{b}{\beta}-\hat{J}(q)\right)\right)^{-1} & 0
\end{array}\right]=\left(\begin{array}{cc}
\left\langle\hat{S}_{q, \omega} \hat{S}_{q,-\omega}\right\rangle & \left\langle\hat{S}_{q, \omega} \hat{\sigma}_{q,-\omega}\right\rangle \\
\left\langle\hat{\sigma}_{q, \omega} \hat{S}_{q,-\omega}\right\rangle & \left\langle\hat{\sigma}_{q, \omega} \hat{\sigma}_{q,-\omega}\right\rangle
\end{array}\right) .
$$


Assim, as funções de auto-correlação e resposta são determinadas para o modelo gaussiano na presença de um campo aleatório. Devemos ainda salientar que o efeito adicionado pela desordem só se faz presente na função de auto-correlação, o que já havia sido previsto e agora foi evidenciado. Interpreta-se o termo extra que surge na auto-correlação como sendo uma memória trazida pelo campo aleatório $H_{r}$. Pode-se dizer que o campo aleatório introduz um comportamento não markoviano em nosso sistema, que previamente possuía essa característica. 


\section{Capítulo 4}

\section{Modelos de spin para mesofases}

\section{cristalinas}

O último tópico abordado nessa dissertação serve como preparação para estudos futuros. Ao contrário dos assuntos precedentes, esse capítulo é um exercício de mecânica estatística, onde mostraremos conexões entre sistemas de spins interagentes e modelos para transições de fase em cristais líquidos. Com essa motivação, fazemos uma pequena introdução a alguns modelos para o estudo de estados da matéria intermediários entre a fase sólida ordenada e a fase líquida isotrópica: cristais plásticos e cristais líquidos. Ainda apresentaremos o modelo de Potts de três estados, estabelecendo uma conexão com um modelo muito simples para a transição nemática em cristais líquidos.

Consideremos um sólido cristalino constituído por grupos moleculares com graus orientacionais de liberdade. Aumentando a temperatura, em geral os ordenamentos translacional e orientacional são perdidos. No entanto, pode ocorrer que um determinado grau de orientação venha a persistir, dependendo da faixa de temperatura e concentração. Quando a simetria translacional é mantida, diz-se que o sistema apresenta uma fase plástico-cristalina, ou seja, os centros de massa dos grupos moleculares que formam essa fase estão distribuídos de forma praticamente uniforme, com simetria translacional, mas suas orientações são aleatórias. No caso do ordenamento orientacional persistir, mas com uma distribuição não uniforme das moléculas, diz-se que surgiu uma fase líquidocristalina. Dessa forma, é possível explicar os termos contraditórios "cristais líquidos" e "cristais plásticos". 
Devido à natureza dessas "mesofases", que em geral são formadas por aglomerados de moléculas, a descrição de suas principais características termodinâmicas se faz por modelos que envolvem acoplamentos entre graus de liberdade translacionais e orientacionais. Cada grupo molecular é designado por uma posição no espaço e ainda são relativamentes orientados segundo um referencial. Sob essa luz, propõe-se um gás de rede com termos de acoplamento entre graus de liberdade orientacionais e translacionais, para descrever as propriedades básicas de um cristal plástico (Galam, 1987). Para um cristal líquido, o modelo de Maier-Saupe oferece um tratamento de campo médio para a transição nemática. Todavia, veremos que nos cristais líquidos, devido à sua estrutura, sua modelagem ocorre de forma mais sutil, definindo um parâmetro de ordem apropriado à descrição das orientações relativas das moléculas (Salinas e Neto, 2005; Gennes e Prost, 1983; Chandrasekhar, 1980). Veremos que um gás de rede de Maier-Saupe seria um modelo adequado para cristais plásticos, porque os grupos moleculares poderiam apontar para qualquer direção da esfera unitária.

Nesse trabalho ainda apresentamos o modelo de Potts de q estados, mostrando seu caráter orientacional, em um espaço vetorial apropriado. A apresentação desse modelo se refere ao contexto do "exercício", quando consideramos a sua versão de três estados do modelo de Potts. O elo se dá no momento que construímos um modelo de Maier-Saupe discretizado com restrições às direções do diretor (que poderá apontar somente nas três direções de um referencial solidário ao laboratório).

\subsection{O gás de rede}

Um modelo de gás de rede foi proposto por Lennard-Jones e Devonshire $(1938,1939)$ para descrever a transição líquido-sólido, mas ganhou destaque com os trabalhos de C. N. Yang e T. D. Lee sobre o fenômeno da condensação (Yang e Lee, 1952a, 1952b). Divide-se o espaço d-dimensional ocupado pelas $N$ moléculas de um dado gás em $V$ células. Cada célula pode acomodar uma única molécula, imitando interações repulsivas de volume excluído. Como esse gás não é ideal, suas moléculas interagem; considerando apenas interações atrativas, a energia do sistema é tanto menor quanto mais próximas as moléculas estiverem umas das outras. O hamiltoniano que descreve esse sistema é dado 
por

$$
\mathcal{H}=-\epsilon \sum_{(i, j)} t_{i} t_{j}
$$

onde a variável $t_{i}$ assume o valor +1 quando uma dada célula acomoda uma molécula, e zero caso contrário, para $i=1,2, \cdots V$; na situação mais simples a soma se dá sobre todos os pares de primeiros vizinhos e a interação entre duas moléculas que ocupam posições adjacentes é mediada por $\epsilon>0$. Como há apenas uma molécula por célula, $\sum_{i=1}^{V} t_{i}=N$, ou seja, a soma de todas a variáveis $t_{i}$ sobre todas as células deve ser igual ao número de moléculas do gás.

Vamos tratar esse sistema na versão de Curie-Weiss, na qual a soma sobre pares de sítios mais próximos é substitída por uma soma sobre todos os possíveis emparelhamentos de uma rede. Fazendo a revisão de algumas propriedades básicas do gás de rede na versão Curie-Weiss, tem-se como ponto inicial a função canônica de partição,

$$
Z=\sum_{\left\{t_{i}\right\}}^{\prime} \exp \left\{\frac{\beta \epsilon}{2 V}\left(\sum_{i} t_{i}\right)^{2}\right\},
$$

onde a soma sobre $\left\{t_{i}\right\}$ deve ser feita sobre todas as configurações das variáveis de spin com o vínculo $\sum_{i=1}^{V} t_{i}=N$. Então temos

$$
Z=\frac{V !}{N !(V-N) !} e^{\frac{\beta \epsilon N^{2}}{2 V}}
$$

Considerando o limite termodinâmico, e usando a expansão de Stirling, podemos escrever a energia livre por partícula,

$$
f(\beta, v)=-\frac{1}{\beta} \lim _{N \rightarrow \infty} \frac{\ln Z}{N}=-\frac{1}{\beta}[v \ln v-(v-1) \ln (v-1)]-\frac{\epsilon}{2 v},
$$

onde $v=V / N$.

Temos então a equação de estado do gás de rede,

$$
\begin{aligned}
p & =-\left(\frac{\partial f}{\partial v}\right)_{T} \\
& =k_{B} T \ln \left(\frac{v}{v-1}\right)-\frac{\epsilon}{2 v^{2}}
\end{aligned}
$$

onde $p$ é a pressão. 
Esse gás de rede deve apresentar um comportamento muito próximo ao do gás de Van der Waals, uma vez que cada molécula ocupa um volume delimitado por uma célula e as partículas se atraem. Para explicitar essa proximidade podemos realizar uma expansão de virial para o gás de rede e comparar com o mesmo tipo de expansão para o gás de Van der Waals. Então

$$
\begin{aligned}
\left.\frac{p}{k_{B} T}\right|_{\text {Van der Waals }} & =\frac{1}{v}+\left(b-\frac{a}{k_{B} T}\right) \frac{1}{v^{2}}+b^{2} \frac{1}{v^{3}}+\cdots, \\
\left.\frac{p}{k_{B} T}\right|_{\text {gás de rede }} & =\frac{1}{v}+\frac{1}{2}\left(1-\frac{\epsilon}{k_{B} T}\right) \frac{1}{v^{2}}+\frac{1}{3 v^{3}}+\cdots .
\end{aligned}
$$

O primeiro termo dessas duas expansões é o termo de gás ideal, e os demais são termos de correção. Na teoria fenomenológica de Van der Waals os parâmetros $a$ e $b$ estão relacionados à atração entre as moléculas de um gás não ideal e à interação de volume excluído, respectivamente. No modelo de gás de rede a impenetrabilidade das moléculas é explícita e a interação atrativa é medida por $\epsilon$. A presença desse paralelismo

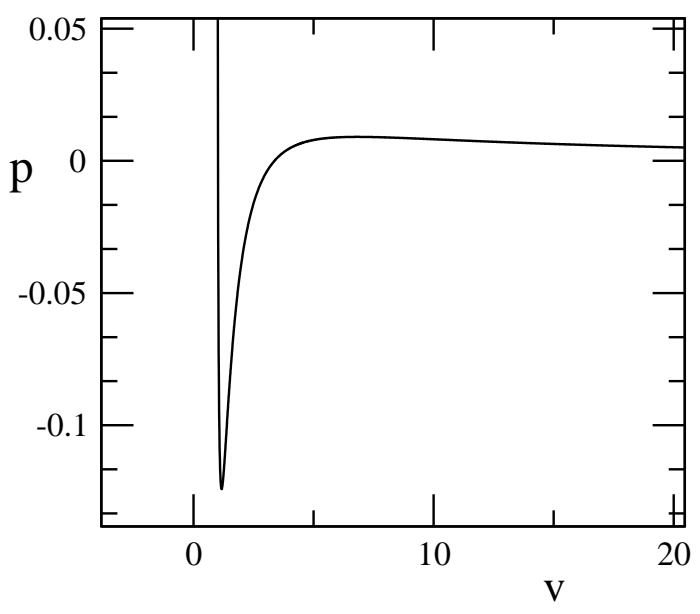
deve se verificar num eventual comportamento crítico. A fim de mostrar as famosas alças de

Figura 4.1: Alças de Van der Waals no gás de rede. Van de Waals numa isoterma $p \times v$, vamos escrever

$$
\frac{d p}{d v}=0=\frac{k_{B} T}{v(v-1)}+\frac{\epsilon}{v^{3}}
$$

A partir dessa relação temos os parâmetros do ponto crítico,

$$
p_{c}=\frac{\epsilon}{8}(2 \ln 2-1), \quad T_{c}=\frac{\epsilon}{4 k_{B}} \quad e \quad v_{c}=\frac{\epsilon}{2 k_{B} T_{c}}=2 .
$$

Além disso, o ponto crítico deve ser um ponto de inflexão, ou seja, $d^{2} p / d v^{2}=0$, como pode ser facilmente verificado. Um exemplo das alças de Van der Waals no gás de rede está na figura 4.1, acima.

\subsubsection{Gás de rede com graus orientacionais de liberdade}

Nesse ponto consideremos um gás de rede em que cada partícula possui uma variável de spin (orientação), surgindo assim acoplamentos entre os graus de liberdade translacionais 
$t_{i}$ e orientacionais (de spin) $\sigma_{i}$. Vamos apresentar uma versão em que temos spins de Ising, embora outras variáveis, como as de Potts (Wu, 1982), poderiam ser usadas (Tsai, 1994). O hamiltoniano a ser considerado é dado por

$$
\mathcal{H}=-\epsilon \sum_{(i, j)} t_{i} t_{j}-J \sum_{(i, j)} t_{i} t_{j} \sigma_{i} \sigma_{j}
$$

onde $t_{i}=0,1 ; \sigma_{i}= \pm 1$, com $i=1, \cdots, V$; sendo $V$ o número de compartimentos de uma rede cristalina. Os parâmetros $\epsilon$ e $J$ são estritamente positivos e as somas são realizadas sobre todos os pares de vizinhos mais próximos.

Fazendo os cálculos no ensemble grande canônico, com número variável de partículas, temos

$$
\begin{aligned}
\Xi & =\sum_{N=0}^{\infty} e^{\beta \mu N} \sum_{\{t\}}^{\prime} \sum_{\{\sigma\}} \exp \left\{\beta \epsilon \sum_{(i, j)} t_{i} t_{j}+\beta J \sum_{(i, j)} t_{i} t_{j} \sigma_{i} \sigma_{j}\right\} \\
& =\sum_{\{t\}} \sum_{\{\sigma\}} \exp \left\{\beta \epsilon \sum_{(i, j)} t_{i} t_{j}+\beta J \sum_{(i, j)} t_{i} t_{j} \sigma_{i} \sigma_{j}+\beta \mu \sum_{i=1}^{V} t_{i}\right\},
\end{aligned}
$$

onde $\sum_{i=1}^{V} t_{i}=N$

Para calcularmos a grande função de partição é interessante realizar uma transformação de variáveis dada por $t_{i} \sigma_{i}=S_{i}=0,0,+1,-1$. Nota-se que $S_{i}^{2}=t_{i}^{2} \sigma_{i}^{2}=t_{i}^{2}=t_{i}$. Levando em conta que o termo $S_{i}=0$ é duplamente degenerado, podemos contar essa degenerescência multiplicando os estados de Gibbs por $2^{\left(1-S_{i}^{2}\right)}$ para cada célula $i$. (É interesante notar que tal modelo possui o mesmo hamiltoniano do modelo de BEG.) Então a grande função de partição é dada por

$$
\Xi=\sum_{\{S\}} \exp \left\{\beta \epsilon \sum_{(i, j)} S_{i}^{2} S_{i}^{2}+\beta J \sum_{(i, j)} S_{i} S_{j}+\beta \mu \sum_{i=1}^{V} S_{i}^{2}+\ln 2 \sum_{i=1}^{V}\left(1-S_{i}^{2}\right)\right\} .
$$

Daqui por diante vamos adotar uma versão de campo médio (ou de Curie-Weiss) para evoluirmos nos cálculos da grande função de partição e encontrar a energia livre de Gibbs por partícula. Na versão do tipo Curie-Weiss temos

$$
\Xi=\sum_{\{S\}} \exp \left\{\frac{\beta \epsilon}{2 V}\left(\sum_{i=1}^{V} S_{i}^{2}\right)^{2}+\frac{\beta J}{2 V}\left(\sum_{i=1}^{V} S_{i}\right)^{2}+(\beta \mu-\ln 2) \sum_{i=1}^{V} S_{i}^{2}+V \ln 2\right\} .
$$

Inserindo uma identidade gaussiana para eliminar os termos quadráticos, temos 


$$
\begin{aligned}
\Xi=\sum_{\{S\}} \int_{-\infty}^{\infty} \frac{d x_{1} d x_{2}}{\pi} \exp \left\{-x_{1}^{2}\right. & -x_{2}^{2}+\frac{\sqrt{2 \beta \epsilon}}{V} x_{1} \sum_{i=1}^{V} S_{1}^{2}+\frac{\sqrt{2 \beta J}}{V} x_{2} \sum_{i=1}^{V} S_{i} \\
& \left.+V \ln 2+(\beta \mu-\ln 2) \sum_{i=1}^{V} S_{i}^{2}\right\} .
\end{aligned}
$$

Calculando a soma sobre as variáveis de spin, temos

$$
\begin{aligned}
\Xi=\int_{-\infty}^{\infty} \frac{d x_{1} d x_{2}}{\pi} \exp \{ & -x_{1}-x_{2}+V \ln 2+V \ln [1+ \\
& \left.\left.+2 \exp \left(\sqrt{\frac{2 \beta \epsilon}{V}} x_{1}+\beta \mu-\ln 2\right) \cosh \left(\sqrt{\frac{2 \beta J}{V}} x_{2}\right)\right]\right\} .
\end{aligned}
$$

Realizando a transformação de variáveis dada por

$$
\sqrt{\frac{2 \beta \epsilon}{V}} x_{1}=\beta \epsilon q \quad e \quad \sqrt{\frac{2 \beta J}{V}} x_{2}=\beta J m,
$$

temos

$$
\Xi=\frac{V \beta}{2 \pi}(J \epsilon)^{2} \int_{-\infty}^{\infty} d q d m e^{-\beta V g(\beta ; q, m)}
$$

onde

$$
g=\frac{\epsilon}{2} q^{2}+\frac{J}{2} m^{2}-\frac{1}{\beta} \ln \left[1+e^{\beta \epsilon q+\beta \mu} \cosh (\beta J m)\right]-\frac{\ln 2}{\beta},
$$

cuja minimização em relação a $q$ e a $m$ fornece a energia livre de Gibbs por partícula desse gás de rede com graus orientacionais de liberdade do tipo Ising.

Uma análise dessa energia livre, muito bem apresentada na ref. (Tsai, 1994), indica que esse modelo possui um diagrama de fase muito rico, com transições de fase de primeira ordem, transições contínuas e com pontos tricríticos. Não iremos prosseguir na análise de estabilidade das soluções paramagnéticas, ferromagnéticas e na construção de um diagrama de fases. Pretendemos apenas comparar o funcional energia livre que acabamos de encontrar com os funcionais dos dois outros modelos apresentados nas duas seções subseqüentes desse último capítulo.

\subsection{O modelo de Potts}

O modelo de Potts é uma generalização do modelo de Ising. No modelo de Ising tem-se dois spins interagentes (primeiros vizinhos) que podem estar em estados paralelos 
ou antiparalelos. A generalização consiste em considerar que cada variável de spin possa assumir $q$ valores diferentes. Assim, a interação entre primeiros vizinhos é $\epsilon_{2}$ caso os dois sítios estejam no mesmo estado e zero caso contrário (Wu, 1982). O hamiltoniano para esse modelo é dado por

$$
\mathcal{H}=-\epsilon_{2} \sum_{(i, j)} \delta_{k r}\left(\sigma_{i}, \sigma_{j}\right)
$$

onde $\epsilon_{2}$ é positivo para o caso ferromagnético e negativo para o caso antiferromagnético; $\sigma_{i}$ é a variável de spin do sítio $i$, podendo assumir $q$ valores no caso geral; $\delta_{k r}\left(\sigma_{i}, \sigma_{j}\right)$ representa o delta de Kronecker, que é 1 quando $\sigma_{i}=\sigma_{j}$ e zero quando $\sigma_{i} \neq \sigma_{j}$.

Segundo $\mathrm{Wu}(\mathrm{Wu}, 1982)$, o modelo de Potts deve ser considerado como um sistema cuja energia depende da orientação relativa entre dois spins de primeiros vizinhos de uma rede, ou seja, depende do ângulo relativo entre os dois spins. Portanto, é interessante escrever a interação tipo delta da última expressão como

$$
\delta_{k r}\left(\sigma_{i}, \sigma_{j}\right)=\frac{1}{q}\left[1+(q-1) \mathbf{e}^{\sigma_{i}} \cdot \mathbf{e}^{\sigma_{j}}\right],
$$

onde $\sigma_{i}=\sigma_{1}, \sigma_{2}, \cdots, \sigma_{q}$, e os $q$ vetores unitários $\mathbf{e}^{\sigma_{i}}$ representam os $q$ estados possíveis de spin. Esses $q$ vetores estão definidos em um espaço de $q-1$ dimensões e apontam nas $q$ dimensões simétricas de um hipertetraedro em $q-1$ dimensões. Para exemplificarmos, tomemos os dois primeiros casos: $q=2$, onde os dois vetores $\mathbf{e}^{\sigma_{1}}$ e $\mathbf{e}^{\sigma_{2}}$ são definidos numa reta e possuem sentido opostos (corresponde ao modelo de Ising); e o caso $q=3$, onde os três vetores $\mathbf{e}^{\sigma_{1}}, \mathbf{e}^{\sigma_{2}}$ e $\mathbf{e}^{\sigma_{3}}$ são definidos no plano e apontam para os três vértices de um triângulo equilátero (figura 4.2). As $q-1$ componentes desses vetores são representadas por $\mathbf{e}_{k}^{\sigma_{i}} \operatorname{com} k=1,2, \cdots, q-1$.

Vamos agora considerar a versão de Curie-Weiss desse modelo para o caso de $q$ estados e em seguida particularizar para o caso $q=3$, cuja solução nos interessa.

Em sua versão de campo médio, consideramos interações de longo alcance entre pares de spins do modelo de Potts, com hamiltoniano descrito por

$$
\mathcal{H}=-\frac{2 \gamma \epsilon_{2}}{N} \sum_{(i<j)} \delta_{k r}\left(\sigma_{i}, \sigma_{j}\right)
$$

onde $N$ é o número de spins e $\gamma$ é o número de coordenação da rede, usando a notação do conhecido trabalho de (Wu, 1982). 


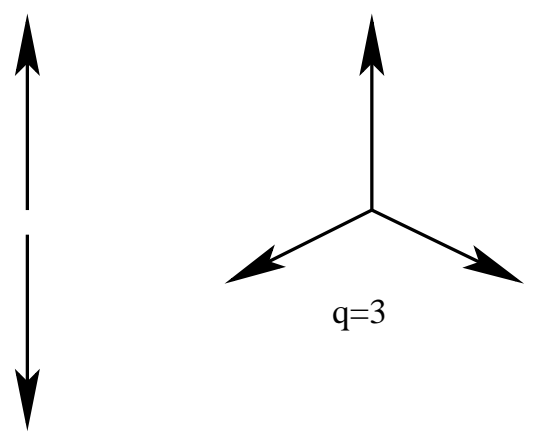

$\mathrm{q}=2$

Figura 4.2: Caso $q=2$ e $q=3$ do modelo de Potts.

Para calcular a função de partição desse modelo devemos usar a relação (4.17). Então escrevemos

$$
Z=\sum_{\{\sigma\}} \exp \left\{\frac{2 \beta \gamma \epsilon_{2}}{N} \sum_{i<j} \frac{1}{q}\left[1+(q-1) \mathbf{e}^{\sigma_{i}} \cdot \mathbf{e}^{\sigma_{j}}\right]\right\}
$$

Após algumas manipulações não é difícil escrever

$$
Z=e^{\beta A} \sum_{\{\sigma\}} \exp \left\{\beta \gamma \epsilon_{2} \frac{q-1}{q N} \sum_{k=1}^{q-1}\left(\sum_{i=1}^{N} \mathbf{e}_{k}^{\sigma_{i}}\right)^{2}\right\}
$$

$\operatorname{com} A=\left(\beta \gamma \epsilon_{2}(n-q)\right) / q$.

Para eliminar os termos quadráticos devemos introduzir $q-1$ identidades gaussianas da forma

$$
e^{a_{k}^{2}}=\int_{-\infty}^{\infty} e^{-x_{k}^{2}+2 a_{k} x_{k}} \frac{d x_{k}}{\sqrt{\pi}}
$$

com

$$
a_{k}=\left(\beta \gamma \epsilon_{2} \frac{q-1}{q N}\right)^{\frac{1}{2}} \sum_{i} \mathbf{e}_{k}^{\sigma_{i}}
$$

Com essa transformação, os termos da função de partição se fatorizam e nós podemos escrever

$$
Z=\frac{e^{\beta A}}{\pi^{\frac{q-1}{2}}} \int_{-\infty}^{\infty} \exp \left\{-\sum_{k=1}^{q-1} x_{k}^{2}+N \ln \left[\sum_{\sigma} \exp \left[2\left(\beta \gamma \epsilon_{2} \frac{q-1}{q N}\right)^{\frac{1}{2}} \sum_{k=1}^{q-1} x_{k} \mathbf{e}_{k}^{\sigma}\right]\right]\right\} \prod_{k=1}^{q-1} d x_{k} .
$$

Considerando ainda a mudança de variáveis

$$
2\left(\beta \gamma \epsilon_{2} \frac{q-1}{q N}\right)^{2} x_{k}=\beta \gamma \epsilon_{2} m_{k}
$$


somos levados à expressão,

$$
Z=C \int_{-\infty}^{\infty} \exp \left\{-N \beta \gamma \epsilon_{2} f(\mathbf{m})\right\} \prod_{k=1}^{q-1} d m_{k}
$$

onde

$$
\begin{aligned}
\mathbf{m} & =\left(m_{1}, m_{2}, \cdots, m_{q-1}\right), \\
C & =e^{\beta A}\left[\frac{N q \beta \gamma \epsilon_{2}}{4 \pi(q-1)}\right]^{\frac{q-1}{N}}
\end{aligned}
$$

$\mathrm{e}$

$$
f(\mathbf{m})=\frac{q}{4(q-1)} \mathbf{m}^{2}-t \ln \left[\sum_{\sigma} \exp \left(\frac{\mathbf{m} \cdot \mathbf{e}^{\sigma}}{t}\right)\right],
$$

na qual fizemos uso de unidades adimensionais, com $t=1 / \beta \gamma \epsilon_{2}$.

A relação acima fornece a energia livre por spin de um modelo de Potts de q estados. Como já foi mencionado, estamos interessados, para nossas comparações futuras, no modelos de 3 estados. Nesse caso devemos considerar o diagrama na figura 4.3, de onde escrevemos

$$
\begin{aligned}
& \mathbf{e}_{x}^{(1)}=1 \quad, \quad \mathbf{e}_{y}^{(1)}=0, \\
& \mathbf{e}_{x}^{(2)}=-\frac{1}{2} \quad, \quad \mathbf{e}_{y}^{(2)}=\frac{\sqrt{3}}{2}, \\
& \mathbf{e}_{x}^{(3)}=-\frac{1}{2} \quad, \quad \mathbf{e}_{y}^{(3)}=-\frac{\sqrt{3}}{2} .
\end{aligned}
$$

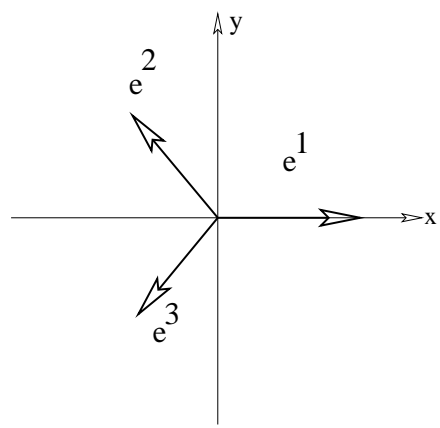

Figura 4.3: Vetores associados ao modelo de Potts de três estados.

Assim, o funcional energia livre do modelo de Potts de três estados é dado por

$$
f\left(t ; m_{1}, m_{2}\right)=\frac{3}{8}\left(m_{1}^{2}+m_{2}^{2}\right)-t \ln \left[e^{\frac{m_{1}}{t}}+2 e^{-\frac{m_{1}}{2 t}} \cosh \left(\frac{\sqrt{3}}{2 t} m_{2}\right)\right] .
$$


Expandindo esse funcional em termos do parâmetro de ordem $\mathbf{m}$, até ordem quatro, verificamos facilmente que o coeficiente do termo cúbico é negativo, indicando uma transição de fase de primeira ordem. Uma análise do diagrama de fase desse modelo não vai ser apresentada. Contudo, tais estudos são encontrados em grande número de fontes (por exemplo (Wu, 1982; Tsai, 1994) e em suas referências).

\subsection{O modelo de Maier-Saupe}

Vamos agora abordar algumas características básicas da formulação de um modelo de campo médio, no sentido de Maier-Saupe, para as transições de fase dos cristais líquidos nemáticos.

Em nossos primeiros passos em ciência aprendemos que a matéria se encontra em três formas: sólida, líquida e gasosa. No entanto, desde o final do século $X I X$ observam-se materiais que apresentam comportamentos intermediários entre as fases sólida e líquida, ou seja, sob alguns aspectos se comportam como sólidos e sob outros como líquidos. Dois exemplos dessas mesofases, como já foi mencionado, são os cristais plásticos e os cristais líquidos. Nos primeiros há uma ordem translacional de longo alcance, sem ordem orientacional de longo alcance entre as moléculas que formam essas estruturas; enquanto os outros são caracterizados por não apresentarem ordem translacional, mas algum tipo de ordenamento orientacional.

As transições para essas fases intermediárias podem ocorrer por processos puramente térmicos (cristais termotrópicos) ou por adição de solventes (cristais liotrópicos). Os representantes mais famosos de materiais nesses estados intermediários da matéria são os cristais líquidos. Uma variedade muito grande de cristais líquidos é conhecida, que por sua vez são separados em classes através de vários critérios que envolvem forma, orientação relativa, propriedades ópticas, etc. Sabe-se ainda que esses materiais são caracterizados por moléculas alongadas, podendo assim ser aproximados, em sua modelagem, por bastonetes.

A nossa análise vai considerar apenas os cristais líquidos nemáticos, que podem ser tanto termotrópicos quanto liotrópicos. Um fluído isotrópico é caracterizado por uma distribuição aleatória dos centros de massas de suas moléculas, bem como pela aleatori- 
edade de suas orientações. Em uma fase nemática, as posições das moléculas ainda são distribuídas aleatoriamente, mas existe uma orientação média entre os bastonetes que representam as moléculas, caracterizada por um diretor $\vec{n}$ (Figura 4.4(a)).

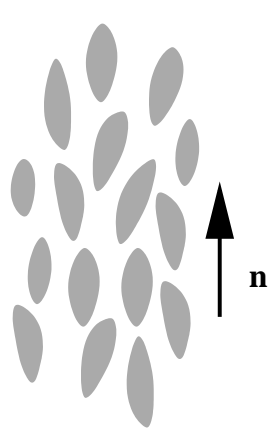

(a)

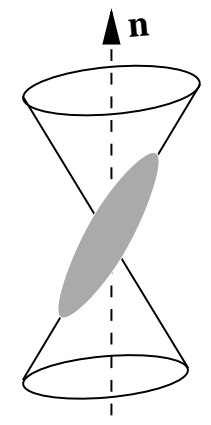

(b)

Figura 4.4: (a) Representação de moléculas, tipo bastonetes, em uma fase nemática; (b) Cada molécula pode assumir qualquer valor de ângulo sólido delimitado pela abertura de um cone.

Fazendo contato com modelos de spins interagentes, um hamiltoniano que descreve as propriedades termodinâmicas de um cristal líquido nemático dependerá das interações entre pares de moléculas, ou seja, da orientação relativa entre duas moléculas na forma de bastonete. O primeiro passo para a construção de tal hamiltoniano consiste em definir um parâmetro de ordem. Consideremos a figura 4.4b; na fase ordenada de um nemático as moléculas apontam, em média, para a direção privilegiada $\mathbf{n}$; em uma fase desordenada não há uma orientação privilegiada. Assim o parâmetro de ordem deve ser nulo na fase desordenada e igual à unidade na fase ordenada. Ao contrário de um sistema de spins, não é possível atribuir um sentido ao eixo maior dessas moléculas alongadas. Portanto, isso vai em sentido oposto a um primeiro pensamento que apontaria para um parâmetro de ordem $S=\cos \theta$, onde $\theta$ é o ângulo entre o eixo maior da molécula e o diretor $\mathbf{n}$. Assim, como as projeções em $\mathbf{n}$ e $-\mathbf{n}$ são equivalentes, deve-se, na verdade, considerar um parâmetro de ordem que envolva $\cos ^{2} \theta$. Uma vez que a média de $\cos ^{2} \theta$ sobre a esfera unitária é $1 / 3$, um parâmetro de ordem cujo valor médio no estado completamente desordenado é zero e 
no estado ordenado é um pode ser dado por

$$
S=P_{2}(\cos \theta)=\frac{1}{2}\left(3 \cos ^{2} \theta-1\right),
$$

onde $P_{2}(\cos \theta)$ é o polinômio de Legendre de segunda ordem. Identificamos assim o parâmetro de ordem $S$ como o momento de quadrupolo. É claro que

$$
\langle S\rangle=\left(3\left\langle\cos ^{2} \theta\right\rangle-1\right) / 2
$$

é igual a zero para $\left\langle\cos ^{2} \theta\right\rangle=1 / 3$ (altas temperaturas) e igual à unidade para $\left\langle\cos ^{2} \theta\right\rangle=1$ (baixas temperaturas).

Nesse ponto podemos passar a nos ocupar do trabalho pioneiro de Maier e Saupe sobre os cristais líquidos. O modelo de Maier-Saupe é o análogo à aproximação de Curie-Weiss para o modelo de Ising e foi originalmente proposto para tratar da transição nemáticoisotrotópica dos cristais líquidos. Aqui vamos dar uma idéia do modelo original e nos referir em seguida a algumas modificações.

Consideremos um par de moléculas na forma de bastonetes, cujos eixos maiores fazem ângulos $\theta_{i}$ e $\theta_{j}$ com respeito ao diretor $\mathbf{n}$ do sistema, que definimos como o eixo $z$ do laboratório. O primeiro termo da energia potencial orientacional entre um par de partículas, obtido por uma expansão multipolar, considerando a simetria cilíndrica, é da forma

$$
u_{i, j}=-A S_{i} S_{j}
$$

onde $S_{i}$ é dado pela relação (4.29) e $A$ é um parâmetro positivo que dependerá de características intrínsecas do sistema. Na versão de campo médio de Maier-Saupe, a interação entre pares é substituída por uma interação de partícula única, sujeita a um campo efetivo de todo o sistema. Nesse enfoque, podemos escrever o hamiltoniano do sistema na forma

$$
\mathcal{H}=-\frac{A}{2 N} \sum_{i, j=1}^{N} S_{i} S_{j},
$$

onde $N$ é o número de partículas. A função de partição canônica associada a esse hamiltoniano é dada por

$$
\begin{aligned}
Z & =\operatorname{Tr} \exp \left\{\frac{\beta A}{2 N}\left(\sum_{i=1}^{N} S_{i}\right)^{2}\right\} \\
& =\int \exp \left\{\frac{\beta A}{2 N}\left(\sum_{i=1}^{N} S_{i}\right)^{2}\right\} \prod_{i=1}^{N} d \Omega_{i},
\end{aligned}
$$


onde $d \Omega_{i}$ é a diferencial de ângulo sólido para a molécula $i$.

Nossos avanços, mais uma vez, estão vinculados à introdução de uma identidade gaussiana, da mesma forma utilizada anteriormente. Feito isso escrevemos

$$
Z=\left(\frac{\beta A N}{2 \pi}\right)^{\frac{1}{2}} \int_{-\infty}^{\infty} d m \exp \{-N \beta A f(m)\}
$$

com

$$
f(m)=\frac{m^{2}}{2}-\frac{1}{\beta A} \ln z
$$

e

$$
z=\int d \Omega \exp \left[\frac{\beta A m}{2}\left(3 \cos ^{2} \theta-1\right)\right]
$$

Em uma primeira análise das características termodinâmicas desse modelo, expandimos o funcional energia livre em potências de $m$. Os primeiros quatro termos dessa expansão são dados por

$$
f(m)=-\frac{\ln 4 \pi}{\beta A}+\frac{1}{2}\left(1-\frac{\beta A}{5}\right) m^{2}-\frac{(\beta A)^{2}}{105} m^{3}+\frac{(\beta A)^{3}}{700} m^{4}+\cdots .
$$

Em concordância com o esquema de Landau, a presença do termo cúbico nessa expansão, com coeficiente negativo, indica transição de fase descontínua. Nessa expressão a temperatura em que o coeficiente do termo quadrático é nulo não se trata de uma temperatura crítica.

\subsubsection{Parâmetro de ordem mais geral}

As observações experimentais mostram que grande parte dos materiais líquidos cristalinos se apresentam em formas menos simétricas, que não seriam representadas pelo parâmetro de ordem dado pela relação (4.29). Tais características estão ligadas à quebra da simetria cilíndrica e à interação de moléculas com diferentes formas geométricas. Em função dessas necessidades, considera-se uma generalização da relação (4.29), dada pelo tensor

$$
Q_{\mu, \nu}^{i, j}=\frac{\lambda}{2}\left(3 i_{\mu} j_{\nu}-\delta_{\mu \nu} \delta_{i j}\right)
$$

onde $\mu, \nu=x, y, z$ se referem aos eixos de um referencial solidário ao laboratório, e $i, j=x^{\prime}, y^{\prime}, z^{\prime}$ a um referencial situado na molécula do cristal líquido; $\lambda_{i}$ está associado à forma da molécula $i$. Ainda em nossa notação $i_{\mu}$ é a projeção do versor i na direção $\mu$ 
e $\delta_{\mu \nu}, \delta_{i j}$ são deltas de Krönecker. Esse tensor é simétrico e de traço nulo, o que garante que será nula a sua média espacial na fase isotrópica.

O tensor acima é representado por uma matriz $3 \times 3$, que pode ser diagonalizada (escrita segundo um referencial adequado), oferecendo

$$
Q_{\mu, \nu}=\left(\begin{array}{ccc}
Q_{11} & 0 & 0 \\
0 & Q_{22} & 0 \\
0 & 0 & Q_{33}
\end{array}\right)
$$

com $Q_{11}+Q_{22}+Q_{33}=0$. Ainda podemos escrever o tensor acima na forma

$$
Q_{\mu, \nu}=S\left(\begin{array}{ccc}
-1 / 2 & 0 & 0 \\
0 & -1 / 2 & 0 \\
0 & 0 & 1
\end{array}\right)
$$

onde $S$ é dado pela relação (4.29).

O conceito de parâmetro de ordem tensorial dado pela relação acima pode ser estendido para descrever uma fase biaxial de um cristal líquido, ou seja, quando a simetria cilíndrica do sistema é perdida. Uma forma bastante útil e geral, para um parâmetro de ordem tensorial simétrico e de traço nulo, que pode expressar a perda de simetria cilíndrica, é dada por

$$
Q_{\mu, \nu}=\left(\begin{array}{ccc}
-\frac{1}{2}(S+\eta) & 0 & 0 \\
0 & -\frac{1}{2}(S-\eta) & 0 \\
0 & 0 & S
\end{array}\right) .
$$

Para $\eta \neq 0, Q_{\mu, \nu}$ descreve uma fase biaxial.

Podemos agora definir um modelo de Maier-Saupe modificado segundo o parâmetro de ordem dado pela relação acima. Consideremos então o hamiltoniano de campo médio das interações orientacionais de um sistema de cristais líquidos nemáticos na forma

$$
\mathcal{H}=-\frac{B}{2 N} \sum_{i, j=1}^{N} \sum_{\mu} S_{\mu \mu}(i) S_{\mu \mu}(j),
$$

onde $B$ é um parâmetro positivo, $N$ é o número de partículas e os termos $S_{\mu \nu}(i), S_{\mu \nu}(j)$ são dados pelos elementos do tensor da equação (4.40). Temos então a função de partição canônica

$$
Z=\operatorname{Tr} \exp \left\{\frac{\beta B}{2 N} \sum_{\mu}\left(\sum_{i=1}^{N} S_{\mu \mu}(i)\right)^{2}\right\}
$$


Usando identidades gaussiana encontramos uma expressão para o funcional energia livre. Segundo a teoria clássica de Landau para as transições de fase, esse funcional energia livre deve ser uma função contínua e lisa do parâmetro de ordem, o que permite realizar uma expansão em torno do ponto em que o parâmetro de ordem se anula. Por argumentos de simetria, uma expansão do funcional energia livre desse modelo apresentará transição de fase de primeira ordem, entre a fase nemática e a fase isotrópica, devido à presença de um termo cúbico. De Gennes utilizou as idéias de Landau para escrever uma expansão dessa natureza em termos dos invariantes do tensor $Q$. No caso de um ferromagneto aparecem potências de expoentes pares do parâmetro de ordem, refletindo a simetria $\pm m$, onde $m$ é a magnetização. No caso de um cristal líquido, aparecerá numa expansão em potências dos invariantes do parâmetro de ordem, ou seja, com termos que não se anulam quando suas médias espaciais são tomadas, que são invariantes por rotação. Considerando a relação (4.37), uma expansão para a energia livre será dada por (Gennes e Prost, 1983; Salinas e Neto, 2005)

$$
F=F_{0}+\frac{1}{2} A(t) \underbrace{Q_{\mu \nu} Q_{\mu \nu}}_{\delta}+\frac{1}{3} B(T) \underbrace{Q_{\mu \varsigma} Q_{\varsigma \kappa} Q_{\kappa \nu}}_{\Delta}+\frac{1}{4} C(T)\left(Q_{\mu \nu} Q_{\mu \nu}\right)^{2}+O\left(Q^{5}\right),
$$

onde, $\delta=Q_{\mu \nu} Q_{\mu \nu}=\sum_{\mu \nu} Q_{\mu \nu} Q_{\mu \nu}$ é a média espacial do quadrado do parâmetro de ordem tensorial.

Mais uma vez, considerando o parâmetro de ordem (4.40), tem-se

$$
\delta=\sum_{\mu}\left(S_{\mu \mu}\right)^{2}=\frac{1}{2}\left(3 S^{2}+\eta^{2}\right)
$$

$\mathrm{e}$

$$
\Delta=\sum_{\mu}\left(S_{\mu \mu}\right)^{3}=\frac{3}{4} S\left(S^{2}+\eta^{2}\right)
$$

Então, toda a análise de estabilidade de modelos para cristais líquidos nemáticos pode ser realizada através de uma expansão da energia livre em termos dos invariantes do parâmetro de ordem, $\delta$ e $\Delta$, de acordo com a teoria de Landau-De Gennes. Não iremos prosseguir com o cálculo da função de partição (4.42), pois nosso interesse reside em investigar uma versão discretizada do modelo de Maier-Saupe, onde o diretor de uma molécula em forma de bastonete pode assumir apenas algumas direções (há a expectativa de que nessa formulação não sejam introduzidas mudanças significativas nas propriedades termodinâmicas). 


\subsubsection{Modelo de Maier-Saupe discretizado}

Consideremos uma molécula $i$ na forma de um bastonete, cuja orientação é dada por um referencial solidário ao laboratório, segundo um vetor que passa por seu eixo maior $\mathbf{n}(i)=\left(n_{x}(i), n_{y}(i), n_{z}(i)\right)$, que é normalizado à unidade. O parâmetro de ordem associado a um sistema de $N$ moléculas idênticas (o que não é obrigatório, pois as formas poderiam ser diferentes, representando uma mistura de diferentes materiais líquido-cristalinos), de acordo com o que já mencionamos, é dado por um tensor quadrupolar de traço nulo,

$$
S_{\mu \nu}(i)=\frac{1}{2} \lambda\left[3 n_{\mu}(i) n_{\nu}(i)-\delta_{\mu \nu}\right]
$$

onde $\mu, \nu=x, y, z$. É interessante notar que o vetor unitário $\mathbf{n}(i)$ é definido sobre o eixo maior da molécula $i$, sem nos referirmos a seu sentido. Ainda devido à simetria do sistema, uma descrição em termos de $\mathbf{n}(i)$ ou $-\mathbf{n}(i)$ deve ser equivalente. Isso é contemplado pelo parâmetro de ordem (4.46), pois ele é invariante sob a mudança $\mathbf{n}(i) \rightarrow-\mathbf{n}(i)$.

Define-se, então, outra forma do modelo de Maier-Saupe,

$$
\mathcal{H}=-\frac{B}{2 N} \sum_{i, j=1}^{N} \sum_{\mu, \nu=x, y, z} S_{\mu \nu}(i) S_{\mu \nu}(j) .
$$

Substituindo a expressão para o parâmetro de ordem (4.46) no hamiltoniano acima, temos

$$
\mathcal{H}=-\frac{B \lambda^{2}}{8 N} \sum_{\mu, \nu}\left[3 \sum_{i=1}^{N} n_{\mu}(i) n_{\nu}(i)-N \delta_{\mu \nu}\right]^{2}
$$

Expandindo o binômio e usando a normalização de $\mathbf{n}(i)$, encontramos

$$
\mathcal{H}=-\frac{B \lambda^{2}}{8 N}\left\{9 \sum_{\mu, \nu}\left[\sum_{i=1}^{N} n_{\mu}(i) n_{\nu}(i)\right]^{2}-3 N^{2}\right\}
$$

A função de partição canônica associada a esse hamiltoniano, é dada por

$$
Z=\operatorname{Tr} \exp \left\{\frac{3 \beta B \lambda^{2}}{8 N}\left[3 \sum_{\mu, \nu}\left(\sum_{i=1}^{N} n_{\mu}(i) n_{\nu}(i)\right)^{2}-N^{2}\right]\right\}
$$

onde o traço representa a soma sobre todas as configurações possíveis dos diretores, ou seja, toda a superfície da esfera unitária. Para simplificarmos os cálculos vamos restringir as possíveis orientações das moléculas, que devem ficar restritas às direções paralelas aos eixos do referencial do laboratório. Essa escolha se baseia no trabalho de Henriques 
e Henriques (Henriques e Henriques, 1997) e no trabalho de Zwanzig sobre o modelo de cilindros rígidos de Onsager. Ainda segundo esses trabalhos, espera-se que essas restrições não alterem resultados qualitativos. Desse maneira, o diretor de uma dada molécula pode ser $\mathbf{n}=( \pm 1,0,0), \mathbf{n}=(0, \pm 1,0)$ ou $\mathbf{n}=(0,0, \pm 1)$.

Com a escolha de Zwanzig, os termos cruzados em $\mu$ e $\nu$ na função de partição serão nulos. Assim escrevemos a função de partição para a versão mais simples do modelo de Maier-Saupe discretizado,

$$
Z=\sum_{\{\mathbf{n}\}} \exp \left\{\frac{3 \beta B \lambda^{2}}{8 N}\left[3 \sum_{\mu}\left(\sum_{i=1}^{N} n_{\mu}^{2}(i)\right)^{2}-N^{2}\right]\right\}
$$

Com essa escolha dos diretores, é interessante verificar uma conexão entre o parâmetro de ordem, dado pela relação (4.46), com a relação (4.40), implicando que

$$
\begin{aligned}
Q_{x x} & =-\frac{1}{2}(S+\eta)=S_{x x}=\frac{1}{2}\left(3 n_{x}^{2}-1\right) \\
Q_{y y} & =-\frac{1}{2}(S-\eta)=S_{y y}=\frac{1}{2}\left(3 n_{y}^{2}-1\right) \\
Q_{z z} & =S=S_{z z}=\frac{1}{2}\left(3 n_{z}^{2}-1\right) .
\end{aligned}
$$

Feitas essas correspondências, após uma pequena álgebra, temos

$$
Z=\sum_{\{\mathbf{S}, \eta\}} \exp \left\{\frac{3 \beta B \lambda^{2}}{8 N}\left[\frac{2}{3}\left(\sum_{i=1}^{N} \eta_{i}\right)^{2}+2\left(\sum_{i=1}^{N} S_{i}\right)^{2}\right]\right\}
$$

Inserindo duas identidades gaussianas, temos

$$
Z=\int_{-\infty}^{\infty} \frac{d \eta^{\prime}}{\sqrt{\pi}} \frac{d S^{\prime}}{\sqrt{\pi}} \exp \left\{-{\eta^{\prime}}^{2}-{S^{\prime}}^{2}\right\}\left\{\sum_{\left\{\eta^{\prime}, S^{\prime}\right\}} \exp \left[\lambda \sqrt{\frac{\beta B}{N}} \eta^{\prime} \eta(i)+\lambda \sqrt{\frac{3 \beta B}{N}} S^{\prime} S(i)\right]\right\}^{N}
$$

Através da relação (4.52) é fácil ver as correspondências entre $\mathbf{n}(i)$ e $(\eta(i), s(i))$,

$$
( \pm 1,0,0) \rightarrow\left(-\frac{3}{2},-\frac{1}{2}\right), \quad(0, \pm 1, o) \rightarrow\left(\frac{3}{2},-\frac{1}{2}\right), \quad(0,0, \pm 1) \rightarrow(0,1) .
$$

Então, sem muito esforço somos levados à forma

$$
\begin{aligned}
Z & =\int_{-\infty}^{\infty} \frac{d \eta^{\prime} d S^{\prime}}{\pi} \exp \left\{-\eta^{\prime 2}-S^{\prime 2}\right. \\
& \left.+N \ln \left[2 \exp \left(\lambda \sqrt{\frac{3 \beta B}{N}} S^{\prime}\right)+4 \exp \left(-\frac{\lambda}{2} \sqrt{\frac{3 \beta B}{N} S^{\prime}}\right) \cosh \left(\frac{3 \lambda}{2} \sqrt{\frac{\beta B}{N}} \eta^{\prime}\right)\right]\right\} .
\end{aligned}
$$


Usando a mudança de variáveis

$$
\sqrt{\frac{3 \beta B}{N}} S^{\prime}=3 \beta B S \quad e \quad \sqrt{\frac{\beta B}{N}}=\beta B \eta
$$

a função de partição para esse modelo de Maier-Saupe discretizado com restrições toma a forma

$$
Z=\mathcal{C} \int_{-\infty}^{\infty} d \eta d S \exp \{-\beta B N f(\beta ; \eta, S)\}
$$

onde $\mathcal{C}=(\beta B N \sqrt{3}) / \pi$, e o funcional energia livre é dado por

$$
f(\beta ; \eta, S)=3 S^{2}+\eta^{2}-t \ln \left[e^{\frac{3 \lambda S}{t}}+2 e^{-\frac{3 \lambda S}{2 t}} \cosh \left(\frac{3 \lambda B \eta}{2 t}\right)\right]-\frac{1}{\beta B} \ln 2,
$$

$\operatorname{com} \beta B=t^{-1}$.

Podemos expandir esse funcional energia livre em potências de $\eta$ e $S$. Tendo em mente a forma dos invariantes do parâmetro de ordem $(\delta$ e $\Delta)$, podemos escrever

$$
f=-t \ln 6+2\left(1-\frac{3 \lambda^{2}}{4 t^{2}}\right) \delta-\frac{3 \lambda^{3}}{2 t^{2}} \Delta+\frac{9 \lambda^{4}}{16 t^{3}} \delta^{3}+\frac{27 \lambda^{5}}{16 t^{4}} \delta \Delta-\frac{117 \lambda^{6}}{320 t^{5}} \delta^{3}+\frac{81 \lambda^{6}}{80 t^{5}} \Delta^{2}+\cdots
$$

Uma vez que o coeficiente do termo cúbico é negativo, temos transição de fase de primeira ordem entre a fase nemática e a fase isotrópica. Nessa expansão aparecem apenas termos com expoentes pares em $\eta$, o que é visto substituindo as expressões dos invariantes do parâmetro de ordem; é justo então que a transição entre as fases uniaxial e biaxial seja de segunda ordem. É possível fazer uma análise minuciosa, que possibilitaria escrever o diagrama de fase desse modelo, encontrando os mínimos dessa energia livre. Uma construção de um diagrama de fase para um modelo de Maier-Saupe, com as mesmas restrições que foram usadas aqui, está em (Henriques e Henriques, 1997), em que se analisa uma mistura líquido-cristalina onde os parâmetros $\lambda_{i}$ obedecem a uma distribuição de probabilidades. Pretendemos em breve estudar modelos para cristais líquidos com desordem numa linha semelhante a este trabalho (Henriques e Henriques, 1997).

\subsubsection{Algumas comparações}

Já foi dito que os cristais plásticos são estudados através de um modelo de gás de rede com graus orientacionais de liberdade. A foma mais elementar de modelar esse sistema consiste em atribuir variáveis de spin do tipo Ising aos grupos moleculares. Porém, um sistema que pode assumir apenas duas orientações está muito distante da realidade. Uma 
generalização consiste em considerar um gás de rede de Potts, ou seja, onde se permite que os grupos moleculares possam apontar segundo q direções diferentes. Na realidade, seria talvez mais realista utilizar um gás de rede com graus de liberdade orientacionais do tipo Maier-Saupe para descrever um cristál plástico genérico. Numa fase posterior, pretendemos explorar esse tipo de gás de rede de Maier-Saupe. Também pretendemos realizar alguns estudos no campo dos fluidos complexos.

Fica assim evidente que esse capítulo aborda as bases de investigações futuras. Mas ainda tráz algumas considerações sobre os modelos estudados. Consideremos os três funcionais energia livre que foram encontrados nessa segunda parte da dissertação,

$$
\begin{gathered}
g_{g . r .}=\frac{\epsilon}{2} q^{2}+\frac{J}{2} m^{2}-\frac{1}{\beta} \ln \left[1+e^{\beta \epsilon q+\beta \mu} \cosh (\beta J m)\right]-\frac{\ln 2}{\beta}, \\
f_{m . P .}=\frac{3}{8}\left(m_{1}^{2}+m_{2}^{2}\right)-t \ln \left[e^{\frac{m_{1}}{t}}+2 e^{-\frac{m_{1}}{2 t}} \cosh \left(\frac{\sqrt{3}}{2 t} m_{2}\right)\right], \\
f_{M-S}=3 S^{2}+\eta^{2}-t \ln \left[e^{\frac{3 \lambda S}{t}}+2 e^{-\frac{3 \lambda S}{2 t}} \cosh \left(\frac{3 \lambda \eta}{2 t}\right)\right]-t \ln 2 .
\end{gathered}
$$

Ao olharmos de relance as três relações acima, num primeiro instante vamos reconhecer que suas estruturas são parecidas. No entanto, não é só isso, pois já foi mencionado que as expansões para cada uma dessas energias livres apresentam um termo cúbico cujo coeficiente é negativo. Mas o mais interessante é a comparação entre a energia livre do modelo de Potts de três estados e a energia livre do modelo de Maier-Saupe discretizado com as restrições nas direções dos diretores. Fazendo a mudança de variáveis, dada por

$$
s=\frac{m_{1}}{\sqrt{8}}, \quad \eta=\sqrt{\frac{3}{8}} m_{2}, \quad e \quad \lambda=\frac{3 \lambda^{\prime}}{\sqrt{8}},
$$

temos

$$
f_{M-S}=\frac{3}{8}\left(m_{1}^{2}+m_{2}^{2}\right)-t \ln \left[e^{\frac{\lambda^{\prime} m_{1}}{t}}+2 e^{-\frac{\lambda^{\prime} m_{1}}{2 t}} \cosh \left(\frac{\sqrt{3} \lambda^{\prime} m_{2}}{2 t}\right)\right]-t \ln 2,
$$

que a menos do fator $\lambda^{\prime}$ e do termo mais à direita é exatamente igual à energia livre do modelo de Potts de três estados.

Essa última expressão não se trata apenas de um resultado curioso, mas também de um indicador da proximidade desses modelos, podendo apontar para novas forma de enxergar 
situações que aparentemente estão desvinculadas. Nesse caso explicitamos a ligação entre modelos de spins interagentes e a descrição de Maier-Saupe para cristais líquidos. 


\section{Capítulo 5}

\section{Considerações finais}

O cerne do presente trabalho foi um estudo bastante geral do modelo gaussiano, explorando suas propriedades termodinâmicas (estáticas) e dinâmicas. No tocante à sua termodinâmica, mostramos que esse modelo não é definido abaixo de sua temperatura crítica, calculamos diversas grandezas, como sua energia livre, energia interna, calor específico. Verificamos o comportamento dessas grandezas nas proximidades da temperatura crítica, analisando também a dimensão crítica. Por fim, num contexto de criticalidade obtivemos a função de correlação de pares na temperatura crítica e em suas proximides.

O estudo da introdução de um comportamento dinâmico no modelo gaussiano foi um pretexto para a apresentação de dois tipos de dinâmicas estocásticas: as dinâmicas de Langevin e a de Cahn-Hilliard. Também procuramos apresentar um novo enfoque para esse tipo de investigação, dado pelo formalismo MSR. Essa técnica inseriu no modelo gaussiano a dinâmica de Langevin de forma diferente, de certa maneira mais física. Mostramos que os resultados para os observáveis de interesse são os mesmos, para um cálculo via MSR e para a aplicação da dinâmica de Langevin diretamente. Como esse formalismo é apropriado ao estudo de sistemas com desordem, estudamos ainda o modelo gaussiano na presença de um campo aleatório.

Por fim, de forma independente, o capítulo quatro se propôs a mostrar evidências das proximidades de três modelos: o modelo de gás de rede com graus de liberdade orientacionais, o modelo de Potts e o modelo de Maier-Saupe para cristais líquidos nemáticos. Para tanto, fizemos uma apresentaçao desses modelos mostrando seu caráter de modelos dependentes de graus de liberdade orientacionais. Na versão de campo médio, também 
calculamos as suas energias livres; notamos que a energia livre de um modelo de Potts de três estados é igual a de um modelo de Maier-Saupe discretizado, mas com restrições às direções em que os diretores podem apontar. Os cálculos do capítulo quarto representam uma motivação para estudos futuros de modelos estatísticos para cristais líquidos. 


\section{Apêndice A}

\section{Introdução elementar aos teoremas}

\section{de flutuação-dissipação (TFD)}

O ensemble canônico, associado a um sistema em equilíbrio termodinâmico com um reservatório a temperatura $T$, é definido pela distribuição de probabilidades

$$
p(\mathcal{C})=\frac{1}{Z} \exp [-\beta E(\mathcal{C})]
$$

onde $\mathcal{C}$ é uma configuração microscópica de energia $E(\mathcal{C}), \beta=1 / k_{B} T$, e

$$
Z=Z(\beta)=\sum_{\mathcal{C}} \exp [-\beta E(\mathcal{C})]
$$

é a função canônica de partição. Portanto, o valor esperado (ou valor médio) de um observável $A$ é dado por

$$
\langle A\rangle=A_{e q}=\frac{1}{Z} \sum_{\mathcal{C}} A(\mathcal{C}) \exp [-\beta E(\mathcal{C})]
$$

Um dos temas pioneiros de Einstein foi a investigação das flutuações, no estado termodinâmico de equilíbrio, dos valores de uma determinada grandeza $A$ em torno de seu valor esperado $\langle A\rangle$. É claro que $\langle\Delta A\rangle=\langle(A-\langle A\rangle)\rangle=0$. Vamos então calcular o valor esperado do segundo momento. Introduzindo uma pequena perturbação acoplada à variável $A, \epsilon=-h A$, podemos escrever a energia de uma configuração $\mathcal{C}$ na forma

$$
E_{\epsilon}(\mathcal{C})=E(\mathcal{C})-h A(\mathcal{C})
$$

É fácil notar que

$$
\langle A\rangle=\frac{1}{\beta Z} \frac{\partial Z}{\partial h}
$$


e que

$$
\left\langle A^{2}\right\rangle-\langle A\rangle^{2}=\frac{1}{\beta^{2}}\left(\frac{1}{Z} \frac{\partial Z}{\partial h}\right)=\frac{1}{\beta} \frac{\partial\langle A\rangle}{\partial h},
$$

que já constitui uma forma do "teorema de flutuação-dissipação" no equilíbrio. Definindo a suscetibilidade (no limite de pequenas perturbações),

$$
\chi_{A}=\left.\frac{\partial\langle A\rangle}{\partial h}\right|_{h=0},
$$

temos outra forma do TFD, bem conhecida no equilíbrio,

$$
\left\langle A^{2}\right\rangle_{0}-\langle A\rangle_{0}^{2}=k_{B} T \chi_{A}
$$

Ainda podemos obter outra expressão do TFD em equilíbrio a partir de uma energia perturbada da forma

$$
E_{\epsilon}(\mathcal{C})=E(\mathcal{C})-h_{A} A\left(\mathcal{C}-h_{B} B(\mathcal{C})\right.
$$

com $h_{A}, h_{B} \rightarrow 0$. Nesse caso é fácil verificar que

$$
\langle A B\rangle_{0}-\langle A\rangle_{0}\langle B\rangle_{0}=k_{B} T \chi_{A, B}
$$

onde

$$
\chi_{A, B}=\left.\frac{\partial\langle A\rangle}{\partial h_{B}}\right|_{h_{A}, h_{B}=0} .
$$

Torna-se então interessante investigar a validade de propriedades desse tipo em situações fora do equilíbrio termodinâmico. De fato, tem havido um esforço considerável nessa direção. Sabe-se, por exemplo, que o análogo dinâmico do TFD continua válido, pelo menos numa forma modificada, contemplando a definição de uma temperatura efetiva (Crisanti e Ritort, 2003; Cugliandolo, 2002; Dominicis e Giardina, 2006).

Vamos então considerar um sistema na presença de uma perturbação dependente do tempo $\epsilon(t)$. Embora haja formalismos mais sofisticados, por exemplo utilizando uma equação de Fokker-Planck ou uma equação mestra, vamos adotar uma abordagem heurística, lançando mão do "princípio de regressão " de Onsager. Inicialmente o sistema está em um estado termodinâmico de equilíbrio $a$; a partir de certo instante é submetido à uma perturbação $\epsilon(t)$, atingindo depois de algum tempo um novo estado de equilíbrio $b$. Segundo Onsager, a evolução a partir do estado de equilíbrio $a$ em direção ao novo estado de equilíbrio $b$ pode ser encarada como uma evolução espontânea de um estado inicial de 
não equilíbrio (ou seja, do estado a) para o estado de equilíbrio natural do sistema (estado b), que é atingido na presença da perturbação $\epsilon(t)$.

A probabilidade canônica de encontrar o sistema no estado termodinâmico $a$, a temperatura $T$, na configuração microscópica $\mathcal{C}_{0}$, é dada por

$$
P\left(\mathcal{C}_{0}\right)=\frac{\exp \left[-\beta E\left(\mathcal{C}_{0}\right)\right]}{\sum_{\mathcal{C}} \exp [-\beta E(\mathcal{C})]}
$$

Introduzimos agora uma pequena perturbação constante, $\epsilon(t)=\epsilon$, a partir do instante inicial $t=0$, associada a um observável $B$. Para $t>0$, a energia do sistema é dada por

$$
E_{\epsilon}(\mathcal{C})=E(\mathcal{C})-\epsilon(t) B(\mathcal{C})
$$

O valor esperado de um observável $A$ será diferente nos estados termodinâmicos inicial (a) e final (b) desse processo. Introduzindo uma probabilidade de transição, o valor médio da grandeza $A$ no estado $b$ pode ser obtido através da relação probabilística

$$
\langle A(t)\rangle_{\epsilon}=\sum_{\mathcal{C C}_{0}} A(\mathcal{C}) P_{\epsilon}\left(\mathcal{C}, t \mid \mathcal{C}_{0}, 0\right) P_{0}\left(\mathcal{C}_{0}\right),
$$

onde $P_{0}\left(\mathcal{C}_{0}\right)$ é a distribuição de probabilidades inicial, no estado não perturbado $a$, e $P_{\epsilon}\left(\mathcal{C}, t \mid \mathcal{C}_{0}, 0\right)$ representa o peso (probabilidade condicional) de todas as possíveis formas de evolução dinâmica que levam da configuração $\mathcal{C}_{0}$ no instante inicial $t=0$ à configuração $\mathcal{C}$ no instante $t$. Na ausência de perturbação, o valor esperado se torna independente do tempo, pois $P_{\epsilon=0}\left(\mathcal{C}, t \mid \mathcal{C}_{0}, 0\right)$ descreve apenas flutuações espontâneas numa situação de equilíbrio macroscópico.

O princípio de Onsager supõe que a probabilidade condicional na presença da perturbação seja dada pela expressão

$$
P_{\epsilon}\left(\mathcal{C}, t \mid \mathcal{C}_{0}, 0\right)=P_{0}\left(\mathcal{C}, t \mid \mathcal{C}_{0}, 0\right) \exp \left\{\beta \epsilon\left[B(\mathcal{C})-B\left(\mathcal{C}_{0}\right)\right]\right\}
$$

ou seja, que as flutuações espontâneas sejam simplesmente corrigidas por fatores adicionais provenientes da perturbação.

Inserindo essa última relação na expressão [A.14] e expandindo até primeira ordem em $\epsilon$, temos

$$
\langle A(t)\rangle_{\epsilon}-\langle A\rangle_{0}=\beta \epsilon\left[\langle A(t) B(t)\rangle_{0}-\langle A(t) B(0)\rangle_{0}\right]
$$


Definindo a função de correlação,

$$
C_{A, B}(t, s)=\langle A(t) B(s)\rangle_{0},
$$

e a função resposta temporal,

$$
\chi_{A, B}(t)=\lim _{\epsilon \rightarrow 0} \frac{\langle A(t)\rangle_{\epsilon}-\langle A\rangle_{0}}{\epsilon},
$$

temos a forma integral do teorema de flutuação-dissipação,

$$
\chi_{A, B}(t)=\beta\left[C_{A, B}(t, t)-C_{A, B}(t, o)\right] .
$$

A forma diferencial do TFD é dada através da função resposta de dois tempos, $R_{A, B}(t, s)$, definida para $t>s$. Então temos

$$
\int_{0}^{t} R_{A, B}(t, s) d s=\chi_{A, B}(t)=\beta \int_{0}^{t} \frac{\partial}{\partial s} C_{A, B}(t, s) d s
$$

conduzindo finalmente à forma diferencial,

$$
R_{A, B}(t, s)=\beta \frac{\partial}{\partial s} C_{A, B}(t, s)
$$

que é válida para $t>s$.

As generalizações do TFD para situações fora do equilíbrio, sobretudo para sistemas com características vítreas, que se encontram em estados de "quase-equilíbrio", vêm indicando que esta última relação deve ser escrita em uma forma ligeiramente diferente,

$$
X_{A, B}(t, s)=\frac{R_{A, B}(t, s)}{\beta \frac{\partial}{\partial s} C_{A, B}(t, s)}, \quad t>s
$$

onde $X_{A, B}(t, s)$ é uma grandeza adimensional, denominada "razão de flutuação-dissipação". No equilíbrio essa razão é igual a um. Para situações de não-equilíbrio, com $X_{A, B}(t, s) \neq 1$, há uma violação do TFD, indicando uma conexão diferente do caso de equilíbrio entre as flutuações térmicas e a dissipação. Costuma-se interpretar o fator $\beta X_{A, B}(t, s)$, como sendo uma temperatura efetiva do sistema. 


\section{Apêndice B}

\section{Integrais Gaussianas}

Iremos fazer aqui uma explanação sucinta do tipo de cálculo presente por trás do processo de obtenção de médias do formalismo MSR. O tratamento apresentado se baseia na obra de Zinn-Justin (Zinn-Justin, 1993).

Passemos a considerar a integral Gaussian generalizada

$$
I(A, b)=\int \exp \left(-\frac{1}{2} \sum_{i, j=1}^{n} x_{i} A_{i j} x_{j}+\sum_{i=1}^{n} b_{i} x_{i}\right) \prod_{i} d x_{i},
$$

em que $A$ é uma matriz simétrica com autovalores $\lambda_{i}$ satisfazendo

$$
R e\left(\lambda_{i}\right) \geq 0 \quad \text { e } \quad \lambda_{i} \neq 0 .
$$

Para calcular $I$ devemos primeiro encontrar o mínimo da forma quadrática

$$
\frac{d}{d x_{k}}\left(\sum_{i, j=1}^{n} \frac{1}{2} x_{i} A_{i j} x_{j}-\sum_{i=1}^{n} b_{i} x_{i}\right)=0,
$$

cuja solução é

$$
x_{i}=\sum_{j}\left(A^{-1}\right)_{i j} b_{j} .
$$

Então, fazendo

$$
x_{i}=\sum_{i}\left(A^{-1}\right)_{i j} b_{j}+y_{i}
$$

e substituindo na relação [B.1], temos

$$
I=\exp \left[\sum_{i, j=1}^{n} \frac{1}{2} b_{i}\left(A^{-1}\right)_{i j} b_{j}\right] \int \exp \left[-\frac{1}{2} \sum_{i, j=1}^{n} y_{i} A_{i j} y_{j}\right] \prod_{i} d y_{i} .
$$


A integral em $y$ da última relação pode ser efetuada através de uma transformação que diagonalize o expoente. A solução de $I$ é, então, dada por

$$
I(A, b)=(2 \pi)^{\frac{n}{2}}(\operatorname{det} A)^{-\frac{1}{2}} \exp \left[\sum_{i, j=1}^{n} \frac{1}{2} b_{i}\left(A^{-1}\right)_{i j} b_{j}\right] .
$$

Vamos agora calcular o l-ésimo momento de um conjunto de variáveis $x_{i}$, distribuídas segundo um peso gaussiano. Esse l-ésimo momento é definido por

$$
\left\langle x_{k_{1}} x_{k_{2}} \cdots x_{k_{l}}\right\rangle \equiv N\left[\prod_{i} \int d x_{i}\right] x_{k_{1}} x_{k_{2}} \cdots x_{k_{l}} \exp \left[-\frac{1}{2} \sum_{i, j=1}^{n} x_{i} A_{i j} x_{j}\right],
$$

onde $N$ é o fator de normalização,

$$
N^{-1}=I(A, o)
$$

Por outro lado, derivando a expressão (B.1) com respeito a $b_{i}$, temos

$$
\frac{\partial}{\partial b_{i}} I(A, b)=\int \exp \left[\frac{1}{2} \sum_{i, j=1}^{n} x_{i} A_{i j} x_{j}+\sum_{i} b_{i} x_{i}\right] \prod_{i} d x_{i} .
$$

Repetindo essa operação $l$ vezes e usando a equação (B.6), somos conduzidos à identidade

$$
\left\langle x_{k_{1}} x_{k_{2}} \cdots x_{k_{l}}\right\rangle=\left.\left\{\frac{\partial}{\partial b_{k_{1}}} \cdots \frac{\partial}{\partial b_{k_{l}}} \exp \left[\frac{1}{2} \sum_{i, j=1}^{n} b_{i}\left(A^{-1}\right)_{i j} b_{j}\right]\right\}\right|_{b=0} .
$$

Esse resultado nos leva a um análogo do teorema de Wick, definido num contexto de teoria quântica de muitos corpos, em celebrado trabalho de de Dominicis et al (Block e Dominicis, 1958). Tal resultado afirma que a média do produto $x_{k_{1}} x_{k_{2}} \cdots x_{k_{l}}$, segundo um peso gaussiano, está relacionado com a inversa da matriz $A$,

$$
\left\langle x_{k_{1}} x_{k_{2}} \cdots x_{k_{l}}\right\rangle=\sum_{\begin{array}{c}
\text { todas as possibilidades de emparelhamento } \\
\text { do conjunto }\left\{k_{1}, \cdots, k_{l}\right\}
\end{array}}\left(A^{-1}\right)_{k_{p_{1}} k_{p_{2}}} \cdots\left(A^{-1}\right)_{k_{p_{l-1}} k_{p_{l}}} \text {. }
$$

Esse resultado é obtido diretamente da equação (B.10), por indução, para um dado $l$ par. 


\section{Referências Bibliográficas}

Amit, D. J. (1993). Field theory, the renormalization group and critical phenomena. World Scientific Publishing.

Berlin, T. H., e Kac, M. (1952). "The spherical model of a ferromagnet". Physical Review, 86, 821-835.

Binney, J. J., Dowrick, N. J., Fisher, A. J., e Newman, M. E. J. (1992). The theory of critical phenomena. Oxford Science Publications.

Block, C., e Dominicis, C. D. (1958). "Un diveloppement du potentiel de gibbs d'un systme quantique composi d'un grand nombre de particules". Nuclear Physics, 7, 459-480.

Cahn, J. W., e Hilliard, L. E. (1958). "Free energy of a nonuniform system. $i$. interfacial free energy". J. Chem. Phys., 28, 258-267.

Carneiro, C. E., e Thomaz, M. T. (2000). "Álgebra dos férmions". Rev. Bras. Ens. de Física, 22, 474-488.

Chaikin, P. M., e Lubensky, T. C. (1995). Principles of condensed matter phisics. Cambridge University Press.

Chandrasekhar, S. (1980). Liquid crystals. Cambridge University Press.

Crisanti, A., e Ritort, F. K. (2003). "Violations of the fluctuation- dissipation theorem in glassy systems: Basic notions and the numerical evidence". J. Phys. A: Math. Gen., 11, 181-290.

Cugliandolo, L. F. (2002). "Dynamics of glassy systems". arxiv:cond-mat/, 0210312. 
Cugliandolo, L. F., e Kurchan, J. (1993). "Analytical solution of the off-equilibrium dynamics of a long-range spin-glass model". Phys. Rev. Lett., 71, 173-176.

Domb, C., e Green, M. S. (1972). Phase transition and critical phenomena (Vol. 2). Academic Press.

Dominicis, C. D. (1976). J. Physique (colloq.), 37, C247.

Dominicis, C. D., e Giardina, I. (2006). Randon fields and spin glasses. Cambridge University Press.

Galam, S. (1987). "Plastic crystals, melting, and randon fields". Phys. Lett. A, 122, 271.

Gennes, P. G. D., e Prost, J. (1983). The physics of liquid crystals (second ed.). Oxford University Press.

Haddad, T. A. S. (2004). Exemplos de universalidade na física estatística de modelos aperiódicos e desordenados. Tese de Doutoramento, IFUSP.

Hase, M. O. (2005). Desordem e competição em modelo de redes - resultados exatos. Tese de Doutoramento, IFUSP.

Henriques, E. F., e Henriques, V. B. (1997). "Biaxial phases is polydisperse mean-field model solutions of uniaxial micelles". J. Chem. Phys., 107, 8036-8040.

Hertz, J. A. (1998). "notas de aula do curso "spin glass physics"”. não publicado, Nordita Dinamarca.

Hohenberg, P. C., e Halperin, B. I. (1977). "Theory of dynamic critical phenomena". Rev. Mod. Phys., 49, 435-479.

Kac, M. (1964). "The work of t. h. berlin in statistical mechanics". Physics Today, 86(10), 40-42.

Kubo, R., Toda, M., e Hashitsume, N. (1985). Statistical physics ii. Springer-Verlag.

Langer, J. S. (1971). "Theory of spinodal decomposition in alloys". Annals of Physics, 65, $53-86$. 
Martin, P. C., Siggia, E., e Rose, H. (1973). "Statistical dynamics of classical systems". Physical Review A, 8, 423.

Salinas, S. R. A. (1997). Introdução à física estatística (second ed.). Edusp.

Salinas, S. R. A., e Neto, A. M. F. (2005). The physics of lyotropic liquid crystals. Oxford University Press.

Tsai, S. H. (1994). Modelo de gás de rede com graus orientacionais de liberdade. Dissertação de Mestrado IFUSP.

Wu, F. Y. (1982). "The Potts model". Rev. Mod. Phys., 54, 235.

Yang, C. N., e Lee, T. D. (1952a). "Statistical theory of equations of state and phase transitions. i. Theory of condensation". Phys. Rev., 87, 404-409.

Yang, C. N., e Lee, T. D. (1952b). "Statistical theory of equations of state and phase transitions. ii. Lattice gas and Ising model". Phys. Rev., 87, 410-419.

Yokoi, C. S. O. (1978). Modelo esférco médio - estudo do comportamento multicrítico a aplicação da técnica de renormalização. Disertação de Mestrado . IFUSP.

Zinn-Justin, J. (1993). Quantum fild theory and critical phenomena (second ed.). Oxford University Press.

Zwanzig, R. (2000). Nonequilibrium statistical mechanics. Oxford University Press. 